\title{
A reduced model for spatially structured predator-prey systems with fast spatial migrations and slow demographic evolutions
}

\author{
François Castella $^{*} \quad$ Jean-Philippe Hoffbeck ${ }^{\dagger} \quad$ Yvan Lagadeuc $^{\ddagger}$
}

\section{Introduction}

In this article we analyze the dynamics of preys and predators evolving both under the effect of spatial migrations, and under the effect of prey-predator interaction. We consider situations where prey-predator demography involves much longer time scales than the faster spatial migrations. We study the effect of spatial heterogenity on the global population dynamics in this regime. In other words, we are here dealing with spatially structured populations, the parameters of the predator-prey dynamics depend on a space variable, and we study how space heterogeneity affects the global demography when migrations are considered fast. The present analysis is strongly motivated by previous works due to J.-C. Poggiale [Po1], [Po2], [Po3], as well as Auger and Poggiale [AP], see below for details.

In the present text, the species repartition depends both on a time variable $t \geq 0$, and on a space variable $x \in \Omega \subset \mathbb{R}^{d}$ (say $d=2$ or $d=3$ ), where $\Omega$ is some bounded domain. On the one hand, we choose to describe prey-predator interaction through a simple LotkaVolterra system: we stress that any other realistic model of prey-predator demography may be analyzed along the similar lines. On the other hand, spatial migrations are described through a linear transfer operator, which conserves the total number of individuals. This is a key point of our analysis: first, migrations which do not preserve the total number of individuals cannot be described along the present lines; second, nonlinear migrations do not directly fall into the scope of the present analysis. These two properties yet correspond to natural ecological assumptions. Indeed, we model migrations caused by the various activities of each individual during one day, possibly in relation with the fact that predation rates, food abundance, etc., differ from one spatial region to another. Taking a linear transfer operator means we assume the transfer rates between the various sites are independent of the populations themselves: the migration of each given individual is not affected by the effective repartition of the global population. Assuming the transfer operator preserves the total number of individuals means that demography and migrations

\footnotetext{
*IRMAR \& IRISA, Université de Rennes 1, Campus de Beaulieu, 35042, Rennes Cedex, France. E-mail : francois.castella@univ-rennes1.fr

†IRMAR, Université de Rennes 1, Campus de Beaulieu, 35042 Rennes Cedex, France \& Lycée du Hainaut, 1 Avenue Villars, 59300 Valenciennes, France. E-mail : jean-philippe.hoffbeck@wanadoo.fr

${ }^{\ddagger}$ UMR CNRS 6553 Ecobio et IFR/FR Caren, Université de Rennes 1, Campus de Beaulieu, 35042 Rennes Cedex, France. E-mail : yvan.lagadeuc@univ-rennes1.fr
} 
are uncorrelated: typically, individuals do not die along migrations. In this context, while the typical time-scale of predator-prey interaction is naturally of the order of several weeks up to one year (it corresponds to the time-scale of the whole population), the time-scale that is relevant for spatial migrations more likely is of the order of several hours up to one day (it corresponds to the time-scale of each individual). As a consequence, the typical ratio between the two scales is small. We denote it by the dimensionless number $\varepsilon>0$ in the sequel.

Summarizing, the present article is devoted to the study of spatially structured predatorprey dynamics of the form

$$
\begin{aligned}
\frac{\partial}{\partial t} \text { (populations) }(t, x)=\frac{1}{\varepsilon} & \text { (effect of migrations })(t, x) \\
& +(\text { effect of predator-prey interaction })(t, x)
\end{aligned}
$$

where all three terms in the above equations naturally depend on both time and space, migrations are modelled through a linear transfer operator, and a simple Lotka-Volterra system is retained for modelling predator-prey interactions, this last choice being made for pure convenience. Note that the number of independent unknowns in (1.1) is parametrized by $x \in \Omega$, so that equation (1.1) is an infinite dimensional dynamical system. Our aim lies in first deriving a reduced model for the above dynamics in the idealized limit $\varepsilon \rightarrow 0$, and second reading off the effect of spatial heterogeneity on the global dynamics in this regime.

Our results are as follows.

We prove that the reduced model is a system of two ordinary differential equations involving the total number of preys resp. predators at time $t$ (regardless of space $x$ ): the detailed dependence of preys resp. predators in the space variable $x$ may be naturally filtered out in the limit $\varepsilon \rightarrow 0$. In other words, both the dependence of the original system (1.1) with respect to the fast time $t / \varepsilon$ (time-scale of the day), and the precise spatial repartition of the populations in the $x$ variable, are ruled out in the reduced model. Furthermore, we prove the limiting equations still are of Lotka-Volterra type, yet with new aggregated coefficients that are obtained upon conveniently averaging both the original coefficients of the Lotka-Volterra part of (1.1), and the coefficients of the linear transfer operator modelling migrations in (1.1). Last, not only are we able to compute the limiting value of model (1.1), but we can also determine corrections to within arbitrary precision of order $\varepsilon^{N}$, for any integer $N$, and even to within exponential precision of order $\exp (-1 / \varepsilon)$. At an informal level, the key property of system (1.1) that leads to such a qualitative behaviour is the following: provided the transfer part of (1.1) involves actual migrations from any site $x$ to any other site $y$ (see below for the precise mixing hypothesis, called irreducibility of the transfer operator in the sequel), then as $\varepsilon \rightarrow 0$, populations tend to go to an equilibrium repartition, where the net effect of migrations eventually vanishes; this prescribes a definite spatial repartition of the populations in the limit $\varepsilon \rightarrow 0$; as a consequence, the only remaining dynamical variable becomes the total amount of preys resp. predators at time $t$, and our result follows. We mention in passing that the similar statements hold true, both at an informal level and in a rigorous fashion, in the case one retains a more sophisticated model than a Lotka-Volterra description to account for preypredator interactions (as we illustrate in an Appendix).

To end this short description of our results, let us mention our analysis shows that, in the present model where a Lotka-Volterra interaction is coupled to fast migrations, spatial heterogeneity is able to explain the destabilization or stabilization of the equilibrium 
state of the original Lotka-Volterra model. In other words, while the uncoupled LotkaVolterra equations have an equilibrium point around which the dynamics exhibit cycles, when perturbed by fast spatial migrations yet, the equations generically break the cycles through either a stabilizing or a destabilizing effect, depending on the very value of the chosen coefficients. This extends a previous observation by Poggiale [Po1].

The present study is directly inspired by previous works due to J.-C. Poggiale [Po1], [Po2], [Po3], as well as Auger and Poggiale [AP]: these works are concerned with the similar question, namely the effect of spatial heterogeneity on global population dynamics in the presence of two different time-scales, yet in the case of finitely many sites (mainly in the case of $N=2$ sites): the starting dynamical system readily is finite dimensional. In that perspective, the present text primarily is a generalization of the above results to the case of continuously many sites, i.e. when the starting dimensional system is infinite dimensional. We also clarify in passing the necessary analysis when the number $N$ of sites is finite but arbitrary.

The key tools that allow for the above quantitative statements are twofolds.

First, the present work requires a detailed analysis of the linear transfer operator involved in (1.1). The important point lies in obtaining an exponential rate of return to equilibrium for solutions of the pure transfer equation

$$
\frac{\partial}{\partial t}(\text { populations })(t, x)=\frac{1}{\varepsilon}(\text { effect of migrations })(t, x),
$$

i.e. our analysis requires the solutions to the pure transfer equation to satisfy an estimate of the form

$$
\forall t \geq 0, \quad \mid(\text { populations })(t, x)-(\text { populations at equilibrium })(x) \mid \leq C \exp \left(-\mu \frac{t}{\varepsilon}\right),
$$

for some universal constants $C>0$ and $\mu>0$. In the finite dimensional setting, such a piece of information may be recovered from the Perron-Frobenius Theorem. In the infinite dimensional case however, the analysis is more delicate. While an estimate of the form (1.2) is easily recovered when the transfer operator satisfies the so-called detailed balance principle (basically a symmetry property that is very natural in the context of statistical physics, but not in the present population dynamics context - see equations (2.16) and (2.17) below for a thorough discussion of this point), we do stress that for populations transfers, the detailed balance principle need by no means be satisfied. For this reason, the spectral analysis of the transfer operator cannot be reduced to that of a symmetric operator, and one needs to analyse the spectrum of a non-self-adjoint operator to establish (1.2). In the present case, our proof of the necessary exponential estimates (1.2) turns out to strongly rely on a previous and quite complete study due to Degond, Goudon, Poupaud [DGP].

The second tool that our approach requires is an appropriate extension of the so-called central manifold theorem in the case of an infinite dimensional system. In the finite dimensional case indeed ([Ca], [Sa]), the central manifold theorem is a powerfull tool to analyze slow-fast systems of the form

$$
\frac{d}{d t} X^{\varepsilon}(t)=f_{0}\left(X^{\varepsilon}(t), Y^{\varepsilon}(t), \varepsilon\right), \quad \frac{d}{d t} Y^{\varepsilon}(t)=\frac{1}{\varepsilon} g_{0}\left(X^{\varepsilon}(t), Y^{\varepsilon}(t), \varepsilon\right),
$$


where the slow-variable is the vector $X^{\varepsilon}(t) \in \mathbb{R}^{M_{x}}$, while the fast-variable is $Y^{\varepsilon}(t) \in \mathbb{R}^{M_{y}}$, for some integers $M_{x}$ and $M_{y}$. We implicitely assume here the functions $f_{0}$ and $g_{0}$ admit expansions of the type $f_{0}(., ., \varepsilon)=f_{0}^{0}(.,)+.\varepsilon f_{0}^{1}(.,)+.\cdots$ and $g_{0}(., ., \varepsilon)=g_{0}^{0}(.,)+.\varepsilon g_{0}^{1}(.,)+$. $\cdots$. The idea is the following: roughly speaking, provided the nonlinear function $g_{0}$ is such that the solutions to the fast system $d Y^{\varepsilon}(t) / d t=\varepsilon^{-1} g_{0}\left(X^{\varepsilon}(t), Y^{\varepsilon}(t), \varepsilon\right)$ satisfy an exponential return to equilibrium property of the form (1.2), the central manifold theorem typically asserts $X^{\varepsilon}(t)$ behaves, as $\varepsilon \rightarrow 0$, as the solution $\widetilde{X^{\varepsilon}}(t)$ to a reduced dynamical system of the form

$$
\frac{d}{d t} \widetilde{X^{\varepsilon}}(t)=f_{0}\left(\widetilde{X^{\varepsilon}}(t), h\left(\widetilde{X^{\varepsilon}}(t), \varepsilon\right), \varepsilon\right)
$$

for some implicit function $h\left(\widetilde{X^{\varepsilon}}(t), \varepsilon\right)$, while the fast variable $Y^{\varepsilon}(t)$ behaves, as $\varepsilon \rightarrow 0$, like $h\left(X^{\varepsilon}(t), \varepsilon\right)$ or equivalently like $h\left(\widetilde{X^{\varepsilon}}(t), \varepsilon\right)$. The error estimates involved in these statements typically have size $\exp (-\mu t / \varepsilon)$. In other words, the original differential system (1.3), posed in $\mathbb{R}^{M_{x}} \times \mathbb{R}^{M_{y}}$, reduces to the lower dimensional system (1.4), posed in $\mathbb{R}^{M_{x}}$, and the stiff dependence of system (1.3) on the fast time variable $t / \varepsilon$ may be averaged out. On top of that, the standard central manifold theorem also asserts one may expand $X^{\varepsilon}(t)$ as $X_{0}(t)+\varepsilon X_{1}(t)+\cdots$, and similarly for $Y^{\varepsilon}(t)$, while variables $X_{0}, X_{1}$, etc. satisfy explicit reduced dynamical systems of the form (1.4). Last, the function $h$ appearing in (1.4) may also be expanded as $h=h_{0}+\varepsilon h_{1}+\cdots$, where $h_{0}, h_{1}$, etc. can be computed explicitely. Upon truncating these expansion to any given order $N$, the obtained error estimates now have typical size $\varepsilon^{N+1}$. In the finite dimensional case, the statement and proof of the central manifold theorem may be found in, e.g. [Ca], or [Sa]. Extensions to the infinite dimensional case may be found in [CL], [VG]. Our approach to proving the necessary infinite dimensional version of the central manifold theorem closely follows the work by Sakamoto [Sa] for finite dimensional systems. An important specifity of Sakamoto's approach which we definitely use is that the central manifold is here constructed close to a trajectory rather than close to a point (the latter construction being more usual). To end this paragraph, note that the original system (1.1) is not in the slow-fast form (1.3) at once, since slow and fast dependences on time are somehow mixed up in the original system (1.1). One key and preliminary step of the analyis is to identify and separate the relevant slow and fast variables in (1.1). This difficulty had already been observed and solved by Poggiale in the above mentionned works, for finitely many (mostly $N=2$ ) sites.

The remaining part of this text is organised as follows.

In section 2, we present the models, both when the number of sites is infinite (sites are indexed by $x \in \Omega$ ), or when it is finite (sites are indexed by $i=1, \ldots, N$ for some $N)$. We thoroughly discuss the models and assumptions, and give a sketch of proof of our asymptotic analysis.

Section 3 gives a complete presentation and proof of a version of the central manifold theorem that is tailored to our needs. The key results here are Theorems 3.1 (existence of a central manifold) and 3.2 (error bounds).

Next, section 4 performs the complete analysis of the discrete dynamics. We first discuss the transfer part of the equations in section 4.1, next apply the central manifold theorem. Our main results are Theorems 4.1 (existence of a spectral gap for the transfer operator), 4.2 (explicit value of the asymptotic dynamics), and 4.3 (error bounds). 
Last, section 5 performs the complete analysis of the continuous dynamics. We first discuss the transfer part of the equations in the long section 5.1, next apply the central manifold theorem again. Our main results are Theorems 5.1 (existence of an equilibrium state for the transfer operator), 5.2 (existence of a spectral gap in $L^{2}$ for the transfer operator), 5.5 (existence of a spectral gap in $C^{0}$ for the transfer operator), 5.7 (explicit value of the asymptotic dynamics), and 5.8 (error bounds).

We end the text with the analysis of two explicit examples (sections 6 and 7 ).

\section{The model}

\subsection{The continuous model}

We choose a compact set $\Omega \subset \mathbb{R}^{d}(d=1,2,3$ in practice), which is the physical space where the given populations of preys and predators evolve. We also take a (small) dimensionless parameter $\varepsilon$, which measures the ratio between the time-scale of migrations and that of prey-predation. Let $p^{\varepsilon}(t, x)$ be the number of preys at time $t \geq 0$ and position $x \in \Omega \subset \mathbb{R}^{d}$, and $q^{\varepsilon}(t, x)$ describe the population of predators at time $t$ and position $x$.

We start with the following system, which is indeed of the form (1.1) discussed in the introduction,

$$
\left\{\begin{aligned}
\frac{\partial p^{\varepsilon}}{\partial t}(t, x)=\frac{1}{\varepsilon} \int_{\Omega}\left[\sigma_{p}(x, y) p^{\varepsilon}(t, y)-\sigma_{p}(y, x) p^{\varepsilon}(t, x)\right] d y & \\
& +\left[a_{p}(x) p^{\varepsilon}(t, x)-b_{p}(x) p^{\varepsilon}(t, x) q^{\varepsilon}(t, x)\right] \\
\frac{\partial q^{\varepsilon}}{\partial t}(t, x)=\frac{1}{\varepsilon} \int_{\Omega}\left[\sigma_{q}(x, y) q^{\varepsilon}(t, y)-\sigma_{q}(y, x) q^{\varepsilon}(t, x)\right] d y & -\left[a_{q}(x) q^{\varepsilon}(t, x)-b_{q}(x) p^{\varepsilon}(t, x) q^{\varepsilon}(t, x)\right] \\
p^{\varepsilon}(0, x)=p_{0}(x), \quad q^{\varepsilon}(0, x) & =q_{0}(x)
\end{aligned}\right.
$$

Here, $p_{0}$ and $q_{0}$ are the (given) repartition of preys resp. predators at the initial time $t=0$. The rate $a_{p}(x)$, assumed independent of time for simplicity, is the $x$-dependent birth rate of preys (in the absence of predators), and $a_{q}(x)$ is the $x$-dependent death rate of predators (in the absence of preys). Similarly, $b_{p}(x)$ and $b_{q}(x)$ represent the death rate of preys due to the presence of predators, resp. the growth rate of the predators' population due to the presence of preys. Naturally, we assume throughout this paper that

$$
\forall x \in \Omega, \quad p_{0}(x) \geq 0, q_{0}(x) \geq 0, a_{p}(x) \geq 0, a_{q}(x) \geq 0, b_{p}(x) \geq 0, b_{q}(x) \geq 0 .
$$

More importantly, we also assume

$$
\left(p_{0}(x), q_{0}(x), a_{p}(x), a_{q}(x), b_{p}(x), b_{q}(x)\right) \in\left(C^{0}(\Omega)\right)^{6} .
$$

Last, the linear transfer operators

$$
\begin{aligned}
K_{p}\left(p^{\varepsilon}\right)(t, x) & :=\int_{\Omega}\left[\sigma_{p}(x, y) p^{\varepsilon}(t, y)-\sigma_{p}(y, x) p^{\varepsilon}(t, x)\right] d y \\
K_{q}\left(q^{\varepsilon}\right)(t, x) & :=\int_{\Omega}\left[\sigma_{q}(x, y) q^{\varepsilon}(t, y)-\sigma_{q}(y, x) q^{\varepsilon}(t, x)\right] d y,
\end{aligned}
$$


have the following meaning. The transfer rate $\sigma_{p}(x, y)$ is the proportion of preys which move from position $y$ to position $x$ at each time, and similarly for $\sigma_{q}(x, y)$. The "+" term in (2.4) accounts for individuals migrating from site $y$ to site $x$, thus increasing the population at site $x$, while the "-" term accounts for individuals migrating from site $x$ to site $y$, decreasing in this way the population at site $x$. Naturally we assume

$$
\forall(x, y) \in \Omega^{2}, \quad \sigma_{p}(x, y) \geq 0, \sigma_{q}(x, y) \geq 0,
$$

as well as

$$
\left(\sigma_{p}(x, y), \sigma_{q}(x, y)\right) \in\left(C^{0}(\Omega \times \Omega)\right)^{2}
$$

For reasons that will become clear later, we shall actually need a reinforced version of (2.6), namely,

$$
\forall(x, y) \in \Omega^{2}, \quad \sigma_{p}(x, y)>0, \sigma_{q}(x, y)>0 .
$$

In other words, for any two sites $x$ and $y$, we assume the rate of transfer from $x$ to $y$ is bounded below by some $\sigma_{\min }>0$. Note in passing the following important property, namely both operators $K_{p}$ and $K_{q}$ are conservative, i.e.

$$
\int_{\Omega} K_{p}\left(p^{\varepsilon}\right)(t, x) d x=0, \quad \int_{\Omega} K_{q}\left(q^{\varepsilon}\right)(t, x) d x=0,
$$

as shown by direct computation. Eq. (2.9) obviously translates the fact that the total number of individuals is kept constant along migrations.

The following basic and preliminary Proposition is easily established.

Proposition 2.1. For any given $\varepsilon>0$, the system (2.1) possesses a unique solution $\left(p^{\varepsilon}(t, x), q^{\varepsilon}(t, x)\right) \in C^{1}\left(\mathbb{R}^{+} ; C^{0}(\Omega) \times C^{0}(\Omega)\right)$. Besides, for any $t \geq 0$, and $x \in \Omega$, one has $p^{\varepsilon}(t, x) \geq 0$ as well as $q^{\varepsilon}(t, x) \geq 0$. Last, the functions $p^{\varepsilon}$ and $q^{\varepsilon}$ are locally bounded in time, uniformly in $\varepsilon$, i.e.

$$
\forall T>0, \exists C_{T}>0, \forall t \in[0 ; T], \forall \varepsilon>0, \quad 0 \leq p^{\varepsilon}(t, x) \leq C_{T}, \quad 0 \leq q^{\varepsilon}(t, x) \leq C_{T} .
$$

Armed with this Proposition, we are in position to analyse the asymptotic behaviour of (2.1) as $\varepsilon \rightarrow 0$. This task is performed in the next sections. More precisely, we shall derive a reduced dynamical system involving the sole total poulations of preys resp. predators ${ }^{1}$,

$$
P_{\mathrm{c}}^{\varepsilon}(t):=\int_{\Omega} p^{\varepsilon}(t, x) d x, \quad Q_{\mathrm{c}}^{\varepsilon}(t):=\int_{\Omega} q^{\varepsilon}(t, x) d x .
$$

Amongst other reasons, this goal can be achieved because the fast part of system (2.1), namely the transfer part, is conservative: the macroscopic variables $P_{\mathrm{c}}^{\varepsilon}$ and $Q_{\mathrm{c}}^{\varepsilon}$ are the only relevant slow variables in the limit $\varepsilon \rightarrow 0$.

Before going further, we here give the proof of Proposition 2.1.

\section{Proof of Proposition 2.1.}

First step (local existence and non-negativity of $p^{\varepsilon}$ and $q^{\varepsilon}$ ).

\footnotetext{
${ }^{1}$ Here and throughout this text, the subscript "c" refers to the continuous model.
} 
Writing the system (2.1) under the form

$$
\left\{\begin{array}{l}
\frac{\partial p^{\varepsilon}}{\partial t}(t, x)=\frac{1}{\varepsilon} K_{p}\left(p^{\varepsilon}\right)(t, x)+F_{p}\left(p^{\varepsilon}(t, x), q^{\varepsilon}(t, x)\right), \\
\frac{\partial q^{\varepsilon}}{\partial t}(t, x)=\frac{1}{\varepsilon} K_{q}\left(q^{\varepsilon}\right)(t, x)+F_{q}\left(p^{\varepsilon}(t, x), q^{\varepsilon}(t, x)\right), \\
p^{\varepsilon}(0, x)=p_{0}(x), \quad q^{\varepsilon}(0, x)=q_{0}(x)
\end{array}\right.
$$

where we define the nonlinear functions

$$
\left\{\begin{array}{l}
F_{p}\left(p^{\varepsilon}(t, x), q^{\varepsilon}(t, x)\right):=\left[a_{p}(x) p^{\varepsilon}(t, x)-b_{p}(x) p^{\varepsilon}(t, x) q^{\varepsilon}(t, x)\right], \\
F_{q}\left(p^{\varepsilon}(t, x), q^{\varepsilon}(t, x)\right):=-\left[a_{q}(x) q^{\varepsilon}(t, x)-b_{q}(x) p^{\varepsilon}(t, x) q^{\varepsilon}(t, x)\right],
\end{array}\right.
$$

it is readily seen that the functions $(p(x), q(x)) \mapsto F_{p}(p(x), q(x))$ and $(p(x), q(x)) \mapsto$ $F_{q}(p(x), q(x))$ acting from $\left[C^{0}(\Omega)\right]^{2}$ to $C^{0}(\Omega)$, are locally Lipschitz. Hence the existence and uniqueness of a local solution $\left(p^{\varepsilon}(t, x), q^{\varepsilon}(t, x)\right) \in C^{1}\left(\left[0, T\left(\varepsilon, p_{0}, q_{0}\right)\right] ;\left[C^{0}(\Omega)\right]^{2}\right)$, defined on some maximal interval $\left[0, T\left(\varepsilon, p_{0}, q_{0}\right)\right)$ which depends on $\varepsilon$ and on the initial data.

The key point is, the functions $p^{\varepsilon}$ and $q^{\varepsilon}$ only take non-negative values. Indeed, equation

$$
\frac{\partial}{\partial t} p^{\varepsilon}(t, x)=\frac{1}{\varepsilon} \int_{\Omega}\left[\sigma_{p}(x, y) p^{\varepsilon}(t, y)-\sigma_{p}(y, x) p^{\varepsilon}(t, x)\right] d y+p^{\varepsilon}(t, x)\left[a_{p}(x)-b_{p}(x) q^{\varepsilon}(t, x)\right]
$$

readily implies, using the Duhamel formula,

$$
\begin{aligned}
& p^{\varepsilon}(t, x)=p_{0}(x) g_{p}^{\varepsilon}(t)+\frac{1}{\varepsilon} \int_{0}^{t} g_{p}^{\varepsilon}(t-s)\left[\int_{\Omega} \sigma_{p}(x, y) p^{\varepsilon}(s, y) d y\right] d s, \\
& \text { where } g_{p}^{\varepsilon}(t):=\exp \left(-\frac{t}{\varepsilon} \int_{\Omega} \sigma_{p}(y, x) d y+\int_{0}^{t}\left[a_{p}(x)-b_{p}(x) q^{\varepsilon}(s, x)\right] d s\right),
\end{aligned}
$$

from which it comes $p^{\varepsilon}(t, x) \geq 0$. The similar argument holds for $q^{\varepsilon}$.

Second step (global existence).

There remains to prove $T\left(\varepsilon, q_{0}, p_{0}\right)=+\infty$.

To do so, we choose an arbitrary time $T>0\left(T<T\left(\varepsilon, p_{0}, q_{0}\right)\right)$, and estimate the functions $p^{\varepsilon}$ and $q^{\varepsilon}$ on $[0, T]$. Using the fact that $K_{p}$ and $K_{q}$ are conservative (2.9), and that the functions $b_{p}, b_{q}, p^{\varepsilon}, q^{\varepsilon}$ only take non-negative values, we estimate the $L^{1}$ norm

$$
\begin{aligned}
\frac{d}{d t} \int_{\Omega} p^{\varepsilon}(t, x) d x & =\int_{\Omega} F_{p}\left(p^{\varepsilon}(t, x), q^{\varepsilon}(t, x)\right) d x \\
& \leq \int_{\Omega} a_{p}(x) p^{\varepsilon}(t, x) \leq\left\|a_{p}\right\|_{L^{\infty}(\Omega)} \int_{\Omega} p^{\varepsilon}(t, x) d x
\end{aligned}
$$

Hence the existence of $C_{T}>0$, which depends on $T$ but not on $\varepsilon$, such that

$$
\forall t \in[0, T], \quad \forall \varepsilon>0, \quad\left\|p^{\varepsilon}(t, x)\right\|_{L^{1}(\Omega)} \leq C_{T} .
$$

Using the equations again, this $L^{1}$ estimate gives in turn an $L^{\infty}$ estimate for $p^{\varepsilon}$. Indeed, we may write, as $t \in[0, T]$,

$$
\begin{aligned}
\frac{\partial}{\partial t} p^{\varepsilon}(t, x) & \leq\left\|\sigma_{p}\right\|_{L^{\infty}\left(\Omega^{2}\right)} C_{T}+a_{p}(x) p^{\varepsilon}(t, x)-b_{p}(x) p^{\varepsilon}(t, x) q^{\varepsilon}(t, x) \\
& \leq\left\|\sigma_{p}\right\|_{L^{\infty}\left(\Omega^{2}\right)} C_{T}+\left\|a_{p}\right\|_{L^{\infty}(\Omega)} p^{\varepsilon}(t, x)
\end{aligned}
$$


where we used the non-negativity of $p^{\varepsilon}$ and $q^{\varepsilon}$. Hence the existence of $C_{T}>0$, independent of $\varepsilon$, such that

$$
\forall(t, x) \in[0, T] \times \Omega, \quad \forall \varepsilon>0, \quad 0 \leq p^{\varepsilon}(t, x) \leq C_{T} .
$$

We are now in position to deduce, using the equation on $q^{\varepsilon}$ together with the non-negativity of $q^{\varepsilon}$ and $p^{\varepsilon}$, the similar $L^{1}$ and $L^{\infty}$ estimates for $q^{\varepsilon}$.

Proposition 2.1 is proved.

\section{$2.2 \quad$ The discrete model}

As we already stressed in the introduction, the fact that (2.1) is an infinite dimensional dynamical systems introduces many difficulties at various stages of the asymptotic analysis. This is the reason why, for sake of completeness and clarity, we also analyze a finite dimensional version of (2.1).

Assume the spatial domain $\Omega$ has been split into $N$ subdomains for some (large) integer $N$, and denote by $p_{i}^{\varepsilon}(t)(i=1, \ldots, N)$ resp. $q_{i}^{\varepsilon}(t)(i=1, \ldots, N)$ the number of preys resp. predators in site number $i$ at time $t$. Then, a reasonable finite dimensional version of (2.1) is

$$
\left\{\begin{array}{l}
\frac{d p_{i}^{\varepsilon}}{d t}(t)=\frac{1}{\varepsilon}\left(\sum_{j=1}^{N}\left[\sigma_{p, i j} p_{j}^{\varepsilon}(t)-\sigma_{p, j i} p_{i}^{\varepsilon}(t)\right]\right)+\left[a_{p, i} p_{i}^{\varepsilon}(t)-b_{p, i} p_{i}^{\varepsilon}(t) q_{i}^{\varepsilon}(t)\right], \\
\frac{d q_{i}^{\varepsilon}}{d t}(t)=\frac{1}{\varepsilon}\left(\sum_{j=1}^{N}\left[\sigma_{q, i j} q_{j}^{\varepsilon}(t)-\sigma_{q, j i} q_{i}^{\varepsilon}(t)\right]\right)-\left[a_{q, i} q_{i}^{\varepsilon}(t)-b_{q, i}(t) p_{i}^{\varepsilon}(t) q_{i}^{\varepsilon}(t)\right], \\
p_{i}^{\varepsilon}(0)=p_{0, i}, \quad q_{i}^{\varepsilon}(0)=q_{0, i} .
\end{array}\right.
$$

Here, the various coefficients $\sigma_{p, i j}, \sigma_{q, i j}, a_{p, i}, a_{q, i}, b_{p, i}, b_{q, i}, p_{0, i}$ and $q_{0, i}$ have the obvious meaning, and extend to the discrete case the notation used in (2.1). We naturally assume

$$
\forall(i, j), \quad \sigma_{p, i j} \geq 0, \sigma_{q, i j}, a_{p, i} \geq 0, a_{q, i} \geq 0, b_{p, i} \geq 0, b_{q, i} \geq 0, p_{0, i} \geq 0, q_{0, i} \geq 0 .
$$

However, we do not assume the transfer coefficients $\sigma_{p, i j}$ or $\sigma_{q, i j}$ be positive, contrary to what is assumed in the continuous case (2.8). This positivity assumption is here replaced by the weaker irreducibility assumption (2.14) which we now introduce.

The matrix of transfer coefficients $\left[\sigma_{p, i j}\right]_{i, j=1}^{N}$ is assumed irreducible.

In other words, for any two sites $i$ and $j, i \neq j$, there exists some integer $r \geq 0$, and a sequence of indices $\left(i, i_{1}, \ldots, i_{r}, j\right)$, possibly reduced to $(i, j)$ in case $r=0$, such that $\sigma_{p, i i_{1}}>0, \sigma_{p, i_{1} i_{2}}>0, \ldots, \sigma_{p, i_{r-1} i_{r}}>0, \sigma_{p, i_{r} j}>0$.

The matrix $\left[\sigma_{q, i j}\right]_{i, j=1}^{N}$ is assumed irreducible as well.

Irreducibility of $\left[\sigma_{p, i j}\right]_{i, j=1}^{N}$ means that for any two given sites $i \neq j$, either $\sigma_{p, i j}>0$, in which case preys may migrate from site $j$ to site $i$ (in this way), or $\sigma_{p, i j}=0$, i.e. the direct transition from $j$ to $i$ is forbidden, yet in this case there are intermediate sites $i_{1}, \ldots, i_{r}$ such that preys may migrate from $j$ to $i_{r}$, next from $i_{r}$ to $i_{r-1}$, and so on, until eventually reaching site $i$. This assumption ensures that migrations really mix the populations of all sites: there is no refuge from which, or to which, migrations are impossible.

We stress irreducibility is an important assumption: there are situations where the matrix of transfer coefficients is neither irreducible, nor can it be split into irreducible 
blocks; in that case, the whole analysis provided in the present text fails. Note however that our analysis anyhow immediately extends to the case where the matrix $\left[\sigma_{p, i j}\right]_{i, j=1}^{N}$ can be split into many irreducible blocks. In this situation, one may split the population of preys into several subpopulations, corresponding to group of sites from which, or to which, preys cannot escape, and each subgroup does satisfy the irreducibility assumption. While in the irreducible case, the asymptotic model only involves the total number of preys, in the case of many ( say $n$ ) independent irreducible blocks at variance, one should instead manipulate the $n$ independent variables given by the $n$ total number of preys belonging to each independent subgroup. Eventually, the whole analysis presented in this text remains unchanged up to writing down as many asymptotic equations as independent groups of preys resp. predators in that case. We shall not dwell on this technical aspect later.

Under the above assumptions, the following Proposition is easily established.

Proposition 2.2. For any given $\varepsilon>0$, the system (2.12) possesses a unique solution $\left(\left\{p_{i}^{\varepsilon}(t)\right\}_{i=1}^{N},\left\{q_{i}^{\varepsilon}(t)\right\}_{i=1}^{N}\right) \in C^{1}\left(\mathbb{R}^{+} ; \mathbb{R}^{2 N}\right)$. Besides, for any $t \geq 0$, and $i \in\{1, \ldots, N\}$, one has $p_{i}^{\varepsilon}(t) \geq 0$ as well as $q_{i}^{\varepsilon}(t) \geq 0$. Last, the vectors $\left(\left\{p_{i}^{\varepsilon}(t)\right\}_{i=1}^{N}\right)$ and $\left(\left\{q_{i}^{\varepsilon}(t)\right\}_{i=1}^{N}\right)$ are locally bounded in time, uniformly in $\varepsilon$, namely

$$
\forall T>0, \exists C_{T}>0, \forall t \in[0 ; T], \forall \varepsilon>0, \forall i \in\{1, \ldots, N\}, \quad 0 \leq p_{i}^{\varepsilon}(t)+q_{i}^{\varepsilon}(t) \leq C_{T} .
$$

Armed with this Proposition, we are in position to analyze the asymptotic behaviour of (2.12). Though the asymptotic analysis of (2.12) is partly performed in [Po1] (at least in the particular case of two sites, i.e. $N=2$ ), we shall completely present the arguments in this text. In doing so we first wish to clarify the parallels and deep differences between the continuous and discrete cases (2.1) resp. (2.12), and second we wish to present at once all the arguments needed in the discrete case: the key ingredient is the Perron-Frobenius theorem. Needless to say, the reduced dynamics obtained in the limit $\varepsilon \rightarrow 0$ again only involves the total populations of preys resp. predators ${ }^{2}$

$$
P_{\mathrm{d}}^{\varepsilon}(t):=\sum_{i=1}^{N} p_{i}^{\varepsilon}(t), \quad Q_{\mathrm{d}}^{\varepsilon}(t):=\sum_{i=1}^{N} q_{i}^{\varepsilon}(t) .
$$

\section{Proof of Proposition 2.2.}

The proof is exactly the same as in the continuous case.

\subsection{Key tools used along the asymptotic analysis}

We end this section with a rough discussion of the mathematical tools we use to analyse both the continuous system (2.1) and the discrete version (2.12).

We begin with the discrete model (2.12). As already noticed by Poggiale, this model may be put in the slow-fast form

$$
\frac{d}{d t} X^{\varepsilon}(t)=f_{0}\left(X^{\varepsilon}(t), Y^{\varepsilon}(t), \varepsilon\right), \quad \frac{d}{d t} Y^{\varepsilon}(t)=\frac{1}{\varepsilon} g_{0}\left(X^{\varepsilon}(t), Y^{\varepsilon}(t), \varepsilon\right),
$$

where the slow resp. fast variables $X^{\varepsilon}(t)$ resp. $Y^{\varepsilon}(t)$ may be explicitely identified. As anticipated above, the natural slow variable here is $\left(P_{\mathrm{d}}^{\varepsilon}(t), Q_{\mathrm{d}}^{\varepsilon}(t)\right) \in \mathbb{R}^{2}$, yet the identification

\footnotetext{
${ }^{2}$ Here and throughout this text, the subscript " $\mathrm{d}$ " refers to the discrete model.
} 
of the correct fast variable is slightly more delicate. Once the system is put in slow-fast form, we are in position to apply the central manifold theorem (see the introduction). In essence, application of this theorem first requires that for any given $X$, solutions $Y=Y(X)$ to the nonlinear equation $g_{0}(X, Y(X), 0)=0$ be uniquely defined: for any given value of the slow variable $X$, the equilibrium value $Y=Y(X)$ of the fast system should be uniquely determined. Second, the linearized function $\left(D g_{0} / D Y\right)(X, Y(X), 0)$ should only possess negative eigenvalues: roughly, the equilibrium values of the equation $g_{0}=0$ are attained exponentially fast in the true dynamical system, i.e. the exponential return to equilibrium property (1.2) should hold. In the finite dimensional setting, both informations (unique equilibrium, which attracts all solutions exponentially) are direct consequences of the Perron-Frobenius theorem for matrices: provided the matrices $\left[\sigma_{p, i j}\right]_{i, j=1}^{N}$ and $\left[\sigma_{q, i j}\right]_{i, j=1}^{N}$ are irreducible (see (2.14)), the equilibrium state is unique and exponentially attracting. As a consequence, our analysis of the discrete model roughly reduces to actually applying the Perron-Frobenius theorem, and to passing to the limit with the help of the central manifold theorem. Incidentally, note that our analysis enables us to expand the natural central manifold to any order in $\varepsilon$. As a consequence, we here derive reduced models for the total populations $\left(P_{\mathrm{d}}^{\varepsilon}(t), Q_{\mathrm{d}}^{\varepsilon}(t)\right)$ which approximate the true dynamics to arbitrary order in $\varepsilon$.

In the continuous case, it turns out a convenient infinite dimensional version of the central manifold theorem needs to be proved anyhow. Our approach is based on an infinite dimensional reformulation of Sakamoto's approach [Sa] to the central manifold theorem. The key information is again the existence of uniquely determined equilibrium states, which should be exponentially attracting. This piece of information requires to precisely analyse the linear transfer operators $K_{p}$ and $K_{q}$ defined in (2.4)-(2.5) (in the relevant functional spaces). The point is, operator $K_{p}$ is not symmetric, nor can it be put in symmetric form. This is where the so-called absence of detailed balance principle enters. In conventional statistical physics indeed, due to the fact that the transition rates $\sigma_{p}(x, y)$ naturally satisfy various symmetry properties (the latter being in turn consequence of thermodynamical first principles), it turns out that the equilibrium states $p_{\text {eq }}(x)$, i.e. the solutions to

$$
K_{p}\left(p_{\text {eq }}\right)(x) \equiv 0,
$$

also necessarily satisfy the detailed balance condition, namely,

$$
\forall(x, y) \in \Omega \times \Omega, \quad \sigma_{p}(x, y) p_{\mathrm{eq}}(y)=\sigma_{p}(y, x) p_{\mathrm{eq}}(x) .
$$

In other words, not only does the integral that defines $K_{p}\left(p_{\text {eq }}\right)$ vanish, but also does the integrand itself. This means that any global equilibrium of the migration operator $K_{p}$, that equilibrates the global effect of populations transitions as a whole, also equilibrates the net fluxes individually between each given sites $x$ and $y$, locally. From a technical point of view, this very strong condition turns out to make $K_{p}$ roughly a self-adjoint operator in that case, so that proving uniqueness of the equilibrium states and exponential attractivity of the latter becomes quite easy. In the present population dynamics context however, the detailed balance principle needs by no means be satisfied. This is where the spectral analysis of $K_{p}$ becomes delicate. In the present text, our analysis of $K_{p}$ borrows many key tools from the text [DGP], from which we deduce the necessary uniqueness and attractivity of the equilibrium states. This allows us to close our analysis. We stress in passing that numerous recent texts have been devoted to the spectral analysis of transfer operators that 
do not satisfy the detailed balance principle, amongst which we wish to quote [Me], [MMP], as well as the review [EM].

Note finally that, apart from the analytical difficulties we point out here, for which the continuous resp. discrete models behave quite differently, the very computations as well as the eventual reduced dynamics obtained both in the continuous and in the discrete case are completely parallel.

\section{A version of the central manifold theorem}

In this section we state a version of the central manifold theorem for dynamical systems whose unknowns belong to a general (infinite dimensional) Banach space. This version is tailored to deal both with the discrete system (2.12) (when put in the relevant slow-fast form (4.11)), and to deal with the infinite dimensional situation (2.1) at once.

\section{Theorem 3.1. (Central Manifold Theorem).}

Let $E$ and $F$ be two Banach spaces and take an integer $r \geq 1$.

Let $f_{0}(x, y, \varepsilon) \in C^{r}(E \times F \times[0,1] ; E)$ and $g_{1}(x, y, \varepsilon) \in C^{r}(E \times F \times[0,1] ; F)$. Take a bounded linear operator $K \in \mathcal{L}(F)$, which is invertible. We assume the following:

(I) the functions $f_{0}, g_{1}$, as well as their derivatives up to order $r$ are bounded.

(II) there are a positive number $\mu>0$ and a constant $C>0$ such that for any $y \in F$, we have

$$
\forall t \geq 0, \quad \forall \varepsilon \in(0,1], \quad\left\|\exp \left(\frac{t}{\varepsilon} K\right) y\right\|_{F} \leq C\|y\|_{F} \exp \left(-\mu \frac{t}{\varepsilon}\right) .
$$

Under the above assumptions, for any $x_{0} \in E, y_{0} \in F, \varepsilon \in(0,1]$, we define $X^{\varepsilon}\left(t, x_{0}, y_{0}\right) \equiv$ $X^{\varepsilon}(t)$ and $Y^{\varepsilon}\left(t, x_{0}, y_{0}\right) \equiv Y^{\varepsilon}(t)$ as the solution for $t \geq 0$ to the differential system

$$
\left(S_{\varepsilon}\right)\left\{\begin{array}{lrl}
\frac{d}{d t} X^{\varepsilon}(t) & =f_{0}\left(X^{\varepsilon}(t), Y^{\varepsilon}(t), \varepsilon\right), & X^{\varepsilon}(0)=x_{0}, \\
\frac{d}{d t} Y^{\varepsilon}(t)=\frac{K}{\varepsilon} Y^{\varepsilon}(t)+g_{1}\left(X^{\varepsilon}(t), Y^{\varepsilon}(t), \varepsilon\right), & Y^{\varepsilon}(0)=y_{0} .
\end{array}\right.
$$

Then, there is an $\varepsilon_{0}>0$ such that for any $\varepsilon<\varepsilon_{0}$, the differential system $\left(S_{\varepsilon}\right)$ possesses a central manifold in the following sense:

(i) there exists a function $h(x, \varepsilon) \in C^{r}\left(E \times\left[0 ; \varepsilon_{0}\right] ; F\right)$ such that for any $\left.\left.\varepsilon \in\right] 0, \varepsilon_{0}\right]$, the set $\mathbf{C}_{\varepsilon}=\{(x, h(x, \varepsilon)) ; x \in E\}$ is invariant under the flow generated by $\left(S_{\varepsilon}\right)$ for $t \geq 0$. Besides, we have $\|h(x, \varepsilon)\|_{L^{\infty}(E ; F)}=O(\varepsilon)$ as $\varepsilon \rightarrow 0$.

(ii) the function $h(x, \varepsilon)$ satisfies the partial differential equation

$$
D_{x} h(x, \varepsilon) f_{0}(x, h(x, \varepsilon), \varepsilon)=\frac{K}{\varepsilon} h(x, \varepsilon)+g_{1}(x, h(x, \varepsilon), \varepsilon),
$$


where $D_{x} h$ stands for $(D h) /(D x)$. On top of that, any bounded function $\widetilde{h}$ such that $\|\widetilde{h}\|_{L^{\infty}} \leq 1,\left\|D_{x} \widetilde{h}\right\|_{L^{\infty}} \leq 1$, and such that we have

$$
D_{x} \widetilde{h}(x, \varepsilon) f_{0}(x, \widetilde{h}(x, \varepsilon), \varepsilon)=\frac{K}{\varepsilon} \widetilde{h}(x, \varepsilon)+g_{1}(x, \widetilde{h}(x, \varepsilon), \varepsilon)+O\left(\varepsilon^{\ell}\right)
$$

in $L^{\infty}$, for some integer $\ell \geq 0$, also necessarily satisfies

$$
\|h-\widetilde{h}\|_{L^{\infty}}=O\left(\varepsilon^{\ell+1}\right) .
$$

(iii) the invariant manifold $\mathbf{C}_{\varepsilon}$ may be approximated in the following sense. There are functions $h_{j}(0 \leq j<r)$, which may be computed recursively, such that for any integer $\ell<r$, we have

$$
\left\|h(x, \varepsilon)-h^{[\ell]}(x, \varepsilon)\right\|_{L^{\infty}(E ; F)}=O\left(\varepsilon^{\ell+1}\right), \text { where } h^{[\ell]}(x, \varepsilon)=\sum_{j=1}^{\ell} \varepsilon^{j} h_{j}(x) .
$$

Remark. The present version of the central manifold Theorem is very close in spirit to the one established in [Sa] for finite dimensional systems: the central manifold is here constructed in the vicinity of the whole trajectory $X^{\varepsilon}(t)$ rather than in the vicinity of the mere origin.

Remark. The apparently very restrictive boundedness assumption on $f_{0}$ and $g_{1}$ is harmless in practice: for given, unbounded functions $f_{0}$ and $g_{1}$, one may alternatively assume that for any given time $T$, the solution $\left(X^{\varepsilon}(t), Y^{\varepsilon}(t)\right)$ of the Theorem satisfies the uniform bound $\left\|X^{\varepsilon}(t)\right\|_{E}+\left\|Y^{\varepsilon}(t)\right\|_{F} \leq C_{T}$ whenever $t \in[0, T]$, where $C_{T}$ does not depend on $\varepsilon$. In that case, a central manifold is constructed as well: it suffices to conveniently truncate $f_{0}$ and $g_{1}$ outside the set $\left\{(x, y) \in E \times F\right.$ s.t. $\left.\|x\|_{E}+\|y\|_{F} \leq C_{T}\right\}$.

Remark. The fact that the sum defining $h^{[\ell]}$ starts from $j=1$ comes from the assumed invertibility of $K$ : at dominant order, the equation $K h_{0}(x)=0$ implies $h_{0} \equiv 0$.

Once the central manifold Theorem is established, one may define the following reduced dynamics, deduced from $\left(S_{\varepsilon}\right)$,

$$
\left(S_{\varepsilon}^{[\infty]}\right) \quad \frac{d}{d t} X^{\varepsilon,[\infty]}(t)=f_{0}\left(X^{\varepsilon,[\infty]}(t), h\left(X^{\varepsilon,[\infty]}(t), \varepsilon\right), \varepsilon\right), \quad Y^{\varepsilon,[\infty]}(t)=h\left(X^{\varepsilon,[\infty]}(t), \varepsilon\right),
$$

where the initial datum is left unspecified for later convenience. As we show below, system $\left(S_{\varepsilon}^{[\infty]}\right)$, supplemented with the appropriate initial datum, approximates the true system $\left(S_{\varepsilon}\right)$ to within exponentially small error terms.

Still under the circumstances of Theorem (3.1), take any integer $\ell<r$, one may define another class of truncated reduced dynamics, called $\left(S_{\varepsilon}^{[\ell]}\right)$, as

$$
\left(S_{\varepsilon}^{[\ell]}\right) \quad \frac{d}{d t} X^{\varepsilon,[\ell]}(t)=f_{0}\left(X^{\varepsilon,[\ell]}(t), h^{[\ell]}\left(X^{\varepsilon,[\ell]}(t), \varepsilon\right), \varepsilon\right), \quad Y^{\varepsilon,[\ell]}(t)=h\left(X^{\varepsilon,[\ell]}(t), \varepsilon\right),
$$

where the initial datum is again left unspecified for later convenience. The dynamics $\left(S_{\varepsilon}^{[\ell]}\right)$ is obtained upon truncating the central manifold to order $\ell$. As we show below, system $\left(S_{\varepsilon}^{[\ell]}\right)$, supplemented with the appropriate initial datum, approximates the true system $\left(S_{\varepsilon}\right)$ to within polynomially small error terms.

The above rough statements are made precise in the next Theorem. 
Theorem 3.2. (error bounds between the reduced models $\left(S_{\varepsilon}^{[\infty]}\right),\left(S_{\varepsilon}^{[\ell]}\right)$, and the original system $\left(S_{\varepsilon}\right)$ ).

Under the assumptions and notation of Theorem 3.1, for any exponant $0<\mu^{\prime}<\mu$, and any initial data $x_{0} \in E, y_{0} \in F$, the following assertions hold true:

(i) (exponential convergence towards the central manifold).

There exists a constant $C>0$ such that

$$
\forall t \geq 0, \quad\left\|Y^{\varepsilon}(t)-h\left(X^{\varepsilon}(t), \varepsilon\right)\right\|_{F} \leq C \exp \left(-\mu^{\prime} \frac{t}{\varepsilon}\right)
$$

(ii) (shadowing principle for $\left(S_{\varepsilon}^{[\infty]}\right)$ ).

For any $T>0$, there exists a perturbed initial data $x_{0}^{\varepsilon}$, implicitely depending on $T$, such that the solution to the reduced system

$$
\left(S_{\varepsilon}^{[\infty]}\right) \quad \frac{d}{d t} X^{\varepsilon,[\infty]}(t)=f_{0}\left(X^{\varepsilon,[\infty]}(t), h\left(X^{\varepsilon,[\infty]}, \varepsilon\right), \varepsilon\right), \quad Y^{\varepsilon,[\infty]}(t)=h\left(X^{\varepsilon,[\infty]}(t), \varepsilon\right),
$$

with initial data $X^{\varepsilon,[\infty]}(0)=x_{0}^{\varepsilon}, Y^{\varepsilon,[\infty]}(0)=h\left(x_{0}^{\varepsilon}, \varepsilon\right)$ satisfies the following error estimate on $[0, T]$

$$
\forall t \in[0, T], \quad\left\|X^{\varepsilon}(t)-X^{\varepsilon,[\infty]}(t)\right\|_{E}+\left\|Y^{\varepsilon}(t)-Y^{\varepsilon,[\infty]}(t)\right\|_{F} \leq C \exp \left(-\mu^{\prime} \frac{t}{\varepsilon}\right) .
$$

where $C>0$ is independent of $t$ and $\varepsilon$. If moreover the solution $X^{\varepsilon}(t)$ to $\left(S_{\varepsilon}\right)$ is bounded on $\mathbb{R}^{+}$, i.e. $\left\|X^{\varepsilon}(t)\right\|_{E} \leq C$ for some $C$ independent of $t \geq 0$ and $\varepsilon$, then one may even take $T=+\infty$ in the above estimates.

(iii) (shadowing principle for $\left(S_{\varepsilon}^{[\ell]}\right)$ ).

Let $x_{0}^{\varepsilon}$ and $T$ be as in the previous item. Choose $\ell \geq 0$. Then, the solution to the truncated reduced system

$$
\left(S_{\varepsilon}^{[\ell]}\right) \quad \frac{d}{d t} X^{\varepsilon,[\ell]}(t)=f_{0}\left(X^{\varepsilon,[\ell]}(t), h\left(X^{\varepsilon,[\ell]}, \varepsilon\right), \varepsilon\right), \quad Y^{\varepsilon,[\ell]}(t)=h^{\ell}\left(X^{\varepsilon,[\ell]}(t), \varepsilon\right)
$$

with initial data $X^{\varepsilon,[\infty]}(0)=x_{0}^{\varepsilon}, Y^{\varepsilon,[\infty]}(0)=h\left(x_{0}^{\varepsilon}, \varepsilon\right)$ satisfies the following error estimate on $[0, T]$

$$
\forall t \in[0, T], \quad\left\|X^{\varepsilon}(t)-X^{\varepsilon,[\ell]}(t)\right\|_{E}+\left\|Y^{\varepsilon}(t)-Y^{\varepsilon,[\ell]}(t)\right\|_{F} \leq C \varepsilon^{\ell+1}+C \exp \left(-\mu^{\prime} \frac{t}{\varepsilon}\right) .
$$

where $C>0$ is independent of $t$ and $\varepsilon$. If moreover the solution $X^{\varepsilon}(t)$ to $\left(S_{\varepsilon}\right)$ is bounded on $\mathbb{R}^{+}$, i.e. $\left\|X^{\varepsilon}(t)\right\|_{E} \leq C$ for some $C$ independent of $t \geq 0$ and $\varepsilon$, then one may even take $T=+\infty$ in the above estimates.

Remark. In other words, the original system $\left(S_{\varepsilon}\right)$, posed in the larger space $E \times F$, is well approximated by either the reduced system $\left(S_{\varepsilon}^{[\infty]}\right)$ or its truncated versions $\left(S_{\varepsilon}^{[\ell]}\right)(\ell \in \mathbb{N})$, posed in the smaller space $E$.

In the case of the population dynamics we are considering here, this statement allows to reduce the original $2 N$ dimensional differential system (2.12) to a two dimensional equation, 
or even the original infinite dimensional differential system (2.1) to a two dimensional equation.

Remark. Note that Theorem 3.2 does not provide a standard error estimate. It rather states a shadowing property. Namely, up to modifying the initial datum $x_{0}$, the solution to $\left(S_{\varepsilon}\right)$ with initial datum $x_{0}$, and the solution to $\left(S_{\varepsilon}^{[\infty]}\right)\left(\operatorname{resp} .\left(S_{\varepsilon}^{[\ell]}\right)\right)$ with initial datum $x_{0}^{\varepsilon}$, coincide to within exponentially small error terms (resp. polynomially small error terms). The modified initial data $x_{0}^{\varepsilon}$ is not uniquely defined in general.

Remark. Again, the boundedness assumption on $f_{0}$ and $g_{1}$ is harmless, see the remark after Theorem 3.1.

We now give the proofs of Theorems 3.1 and 3.2. We begin with the

\section{Proof of Theorem 3.2.}

The proof of point (i) is easy. Indeed, we have on the one hand

$$
\frac{d}{d t} Y^{\varepsilon}(t)=\frac{K}{\varepsilon} Y^{\varepsilon}(t)+g_{1}\left(X^{\varepsilon}(t), Y^{\varepsilon}(t), \varepsilon\right)
$$

while on the other hand

$$
\frac{d}{d t} h\left(X^{\varepsilon}(t), \varepsilon\right)=D_{X} h\left(X^{\varepsilon}(t), \varepsilon\right) f_{0}\left(X^{\varepsilon}(t), h\left(X^{\varepsilon}(t), \varepsilon\right), \varepsilon\right) .
$$

The point is, invariance of the central manifold $Y=h(X, \varepsilon)$ under the forward flow of $\left(S_{\varepsilon}\right)$ implies the identity

$$
D_{X} h(X, \varepsilon) f_{0}(X, h(X, \varepsilon), \varepsilon)=\frac{K}{\varepsilon} h(X, \varepsilon)+g_{1}(X, h(X, \varepsilon), \varepsilon),
$$

for any $X \in E$. As a consequence, we recover in (3.2)

$$
\frac{d}{d t} h\left(X^{\varepsilon}(t), \varepsilon\right)=\frac{K}{\varepsilon} h\left(X^{\varepsilon}(t), \varepsilon\right)+g_{1}\left(X^{\varepsilon}(t), h\left(X^{\varepsilon}(t), \varepsilon\right), \varepsilon\right) .
$$

Now applying the Duhamel formula to the two differential equations (3.1) and (3.3) gives

$$
\begin{aligned}
Y^{\varepsilon}(t) & -h\left(X^{\varepsilon}(t), \varepsilon\right)=\exp \left(t \frac{K}{\varepsilon}\right)\left[y_{0}-h\left(x_{0}, \varepsilon\right)\right] \\
& +\int_{0}^{t} \exp \left((t-s) \frac{K}{\varepsilon}\right)\left[g_{1}\left(X^{\varepsilon}(s), Y^{\varepsilon}(s), \varepsilon\right)-g_{1}\left(X^{\varepsilon}(s), h\left(X^{\varepsilon}(s), \varepsilon\right), \varepsilon\right)\right] d s
\end{aligned}
$$

Hence, using the fact $g_{1}$ is globally Lipschitz, together with the assumed exponential smallness of $\exp (t K / \varepsilon)$, we recover

$$
\begin{gathered}
\left\|Y^{\varepsilon}(t)-h\left(X^{\varepsilon}(t), \varepsilon\right)\right\|_{F} \leq C \exp \left(-\mu \frac{t}{\varepsilon}\right)\left\|y_{0}-h\left(x_{0}, \varepsilon\right)\right\|_{F} \\
+C \int_{0}^{t} \exp \left(-(t-s) \frac{\mu}{\varepsilon}\right)\left\|Y^{\varepsilon}(s)-h\left(X^{\varepsilon}(s), \varepsilon\right)\right\|_{F} d s .
\end{gathered}
$$

The Gronwall Lemma applied to the function $\exp (+\mu t / \varepsilon)\left|Y^{\varepsilon}(t)-h\left(X^{\varepsilon}(t), \varepsilon\right)\right|$ gives the estimate (and any exponant $\mu^{\prime}=\mu-C \varepsilon$ is allowed). 
The proof of point (iii) in turn is fairly easy once point (ii) is proved. This comes from the fact that

$$
h(x, \varepsilon)=h^{[\ell]}(x, \varepsilon)+O\left(\varepsilon^{\ell+1}\right),
$$

uniformly with respect to $x \in E$.

Hence, there only remains to prove point (ii). To do so, let us take any $T>0$, and introduce the unique solution $X_{T}^{\varepsilon,[\infty]}(t)$ to the reduced system $\left(S_{\varepsilon}^{[\infty]}\right)$ with initial data given at time $T$

$$
X_{T}^{\varepsilon,[\infty]}(T)=X^{\varepsilon,[\infty]}(T) .
$$

Using the (already proved) fact that $Y^{\varepsilon}(t)=h\left(X^{\varepsilon}(t), \varepsilon\right)+O\left(\exp \left(-\mu^{\prime} t / \varepsilon\right)\right)$, we may write

$$
\begin{aligned}
& \frac{d}{d t} X^{\varepsilon}(t)=f_{0}\left(X^{\varepsilon}(t), h\left(X^{\varepsilon}(t), \varepsilon\right)+O\left(\exp \left(-\mu^{\prime} \frac{t}{\varepsilon}\right)\right), \varepsilon\right), \\
& \frac{d}{d t} X_{T}^{\varepsilon,[\infty]}(t)=f_{0}\left(X_{T}^{\varepsilon,[\infty]}(t), h\left(X_{T}^{\varepsilon,[\infty]}, \varepsilon\right), \varepsilon\right) .
\end{aligned}
$$

Integrating these equations backwards from $T$ to $t$, we recover

$$
\begin{aligned}
\left\|X^{\varepsilon}(t)-X_{T}^{\varepsilon,[\infty]}(t)\right\|_{E} & \\
\leq & \int_{t}^{T} \| f_{0}\left(X^{\varepsilon}(s), h\left(X^{\varepsilon}(s), \varepsilon\right)+O\left(\exp \left(-\mu^{\prime} \frac{s}{\varepsilon}\right)\right), \varepsilon\right) \\
& \quad-f_{0}\left(X_{T}^{\varepsilon,[\infty]}(s), h\left(X_{T}^{\varepsilon,[\infty]}(t), \varepsilon\right), \varepsilon\right) \|_{E} d s \\
\leq & C \int_{t}^{T}\left[\left\|X^{\varepsilon}(s)-X_{T}^{\varepsilon,[\infty]}(s)\right\|_{E}+O\left(\exp \left(-\mu^{\prime} \frac{s}{\varepsilon}\right)\right)\right] d s
\end{aligned}
$$

where we have used the fact that $f_{0}$ and $h$ are globally Lipschitz. From this we deduce that, for any $0<\mu^{\prime \prime}<\mu^{\prime}$, we have

$$
\forall 0 \leq t \leq T, \quad\left\|X^{\varepsilon}(t)-X_{T}^{\varepsilon,[\infty]}(t)\right\|_{E} \leq C \exp \left(-\mu^{\prime \prime} \frac{t}{\varepsilon}\right)
$$

This ends the proof of point (ii) in case $T<\infty$.

Now, in the particular case when $X^{\varepsilon}(t)$ is uniformly bounded on $\mathbb{R}^{+}$, namely $\forall t \geq 0$, $\left\|X^{\varepsilon}(t)\right\|_{E} \leq C$ for some $C$ independent of $t$ and $\varepsilon$, the above considerations show that $X_{T}^{\varepsilon,[\infty]}$ is bounded independently of $\varepsilon, t$, and $T$, and, thanks to the differential equation satisfied by $X_{T}^{\varepsilon,[\infty]}$, so does $d X_{T}^{\varepsilon,[\infty]} / d t$. Ascoli's Theorem thus allows to exhibit a limit of $X_{T}^{\varepsilon,[\infty]}$ as $T \rightarrow \infty$, the limit being uniform on compact subintervals of $\mathbb{R}^{+}$. Passing to the limit in (3.4) as $T \rightarrow \infty$ shows one can indeed take $T=+\infty$ in statement (ii) under these circumstances.

This ends the proof of Theorem 3.2.

\section{Proof of Theorem 3.1.} $[\mathrm{Ca}]$.

We closely follow the standard proof of the 'usual' central manifold theorem, see e.g. 
Throughout this proof, let $M$ be a common bound for $f_{0}, g_{1}$, as well as all their derivatives up to order $r$.

Proof of point (i).

Define

$$
B:=\left\{h: E \rightarrow F \text { s.t. } h \text { is } C^{1} \text { and }\|h\|_{L^{\infty}}(E ; F) \leq 1,\left\|D_{x} h\right\|_{L^{\infty}(E ; \mathcal{L}(E ; F))} \leq 1\right\},
$$

where $D_{x} h$ denotes $(D h) /(D x)$. On the other hand, given $h \in B$, and given $x_{0}$ in $E$, define $X\left(t, x_{0}, h, \varepsilon\right)$, also denoted by $X_{h}(t)$ in the sequel, as the solution to

$$
\begin{aligned}
& \frac{d}{d t} X\left(t, x_{0}, h, \varepsilon\right)=f_{0}\left(X\left(t, x_{0}, h, \varepsilon\right), h\left(X\left(t, x_{0}, h, \varepsilon\right)\right), \varepsilon\right), \\
& X\left(t, x_{0}, h, \varepsilon\right)=x_{0} .
\end{aligned}
$$

In turn, define $Y\left(t, x_{0}, h, \varepsilon\right)$, also denoted by $Y_{h}(t)$ in the sequel, as

$$
Y\left(t, x_{0}, h, \varepsilon\right)=\exp \left(+\frac{t}{\varepsilon} K\right) \int_{-\infty}^{t} \exp \left(-\frac{s}{\varepsilon} K\right) g_{1}\left(X\left(s, x_{0}, h, \varepsilon\right), h\left(X\left(s, x_{0}, h, \varepsilon\right)\right), \varepsilon\right) d s .
$$

In other words, $Y\left(t, x_{0}, h, \varepsilon\right)$ satisfies

$$
\begin{aligned}
& \frac{d}{d t} Y\left(t, x_{0}, h, \varepsilon\right)=\frac{K}{\varepsilon} Y\left(t, x_{0}, h, \varepsilon\right)+g_{1}\left(X\left(t, x_{0}, h, \varepsilon\right), h\left(X\left(t, x_{0}, h, \varepsilon\right)\right), \varepsilon\right), \\
& Y\left(t, x_{0}, h, \varepsilon\right) \text { remains bounded as } t \rightarrow+\infty .
\end{aligned}
$$

Last, define the operator $T$ as

$$
T h\left(x_{0}\right):=Y\left(0, x_{0}, h, \varepsilon\right)=\int_{-\infty}^{0} \exp \left(-\frac{s}{\varepsilon} K\right) g_{1}\left(X\left(s, x_{0}, h, \varepsilon\right), h\left(X\left(s, x_{0}, h, \varepsilon\right)\right), \varepsilon\right) d s .
$$

It is clear that any fixed point of map $T$, i.e. any function $h$ such that $T h\left(x_{0}\right)=h\left(x_{0}\right)$ for any $x_{0}$, is a central manifold for the original system $\left(S_{\varepsilon}\right)$.

We now show $T$ has a unique fixed point in $B$, proving in this way the existence of a central manifold for $\left(S_{\varepsilon}\right)$.

First, it is easily seen that $T$ maps $B$ into itself for small enough $\varepsilon$. Indeed, we clearly have, using assumption (II) of the Theorem,

$$
\left\|T h\left(x_{0}\right)\right\|_{L^{\infty}} \leq \int_{-\infty}^{0} \exp \left(+\mu \frac{s}{\varepsilon}\right) M d s=\frac{M \varepsilon}{\mu},
$$

a quantity which is $\leq 1$ for small enough $\varepsilon$. Besides, we have

$$
\begin{aligned}
& \frac{d}{d t} D_{x_{0}} X_{h}(t)=D_{x} f_{0}\left(X_{h}(t), h\left(X_{h}(t)\right), \varepsilon\right) D_{x_{0}} X_{h}(t) \\
& \quad+D_{y} f_{0}\left(X_{h}(t), h\left(X_{h}(t)\right), \varepsilon\right) D_{x} h\left(X_{h}(t)\right) D_{x_{0}} X_{h}(t), \quad D_{x_{0}} X_{h}(t)=\mathrm{Id}
\end{aligned}
$$


from which Gronwall's Lemma allows to deduce

$$
\left\|D_{x_{0}} X_{h}(t)\right\|_{L^{\infty}} \leq \exp (2 M t)
$$

(the $L^{\infty}$ norm is taken with respect to the $x_{0}$ dependence of $X_{h}(t)$ ), and one may eventually write, with obvious shorthand notation,

$$
\begin{aligned}
\left\|D_{x_{0}} T h\left(x_{0}\right)\right\|_{L^{\infty}} & \leq \int_{-\infty}^{0} \exp \left(+\mu \frac{s}{\varepsilon}\right)\left\|D_{x} g_{1} D_{x_{0}} X_{h}(s)+D_{y} g_{1} D_{x} h D_{x_{0}} X_{h}(s)\right\| d s \\
& \leq \int_{-\infty}^{0} \exp \left(+\mu \frac{s}{\varepsilon}\right) 2 M \exp (-2 M s) d s \\
& =\frac{2 M \varepsilon}{\mu-2 M \varepsilon},
\end{aligned}
$$

a quantity which is $\leq 1$ for small enough $\varepsilon$.

The similar estimates allow to prove $T$ is a contraction on $B$. Indeed, we may write for instance, taking two functions $h_{1}$ and $h_{2}$ in $B$,

$$
\left\|\frac{d}{d t}\left(X_{h_{1}}(t)-X_{h_{2}}(t)\right)\right\|_{L^{\infty}} \leq M\left\|X_{h_{1}}(t)-X_{h_{2}}(t)\right\|_{L^{\infty}}+M\left\|h_{1}-h_{2}\right\|_{L^{\infty}},
$$

implying

$$
\left\|X_{h_{1}}(t)-X_{h_{2}}(t)\right\|_{L^{\infty}} \leq M t \exp (M t)\left\|h_{1}-h_{2}\right\|_{L^{\infty}},
$$

so that

$$
\begin{aligned}
\left\|T h_{1}-T h_{2}\right\|_{L^{\infty}} & \leq \int_{-\infty}^{0} \exp \left(+\mu \frac{s}{\varepsilon}\right)\left(2 M\left\|X_{h_{1}}(s)-X_{h_{2}}(s)\right\|_{L^{\infty}}+M\left\|h_{1}-h_{2}\right\|_{L^{\infty}}\right) d s \\
& \leq \int_{-\infty}^{0} \exp \left(+\mu \frac{s}{\varepsilon}\right) 2 M^{2}|s| \exp (-M s)\left\|h_{1}-h_{2}\right\|_{L^{\infty}}+M\left\|h_{1}-h_{2}\right\|_{L^{\infty}} \\
& \leq \frac{2 M^{3}+M}{\mu-\varepsilon M} \varepsilon,
\end{aligned}
$$

a quantity which is smaller than one for $\varepsilon$ small enough. One proves in the similar way that

$$
\left\|D_{x_{0}}\left(T h_{1}\left(x_{0}\right)-T h_{2}\left(x_{0}\right)\right)\right\|_{L^{\infty}} \leq C(M, \mu, \varepsilon) \varepsilon\left(\left\|h_{1}-h_{2}\right\|_{L^{\infty}}+\left\|D_{x}\left(h_{1}-h_{2}\right)\right\|_{L^{\infty}}\right),
$$

for some $C(M, \mu, \varepsilon)$ which is bounded as $\varepsilon \in[0,1]$.

All this establishes that $T$ is a contraction on $B$, hence $T$ possesses a unique fixed point $h=h\left(x_{0}, \varepsilon\right) \in B$ for $\varepsilon$ small enough.

To prove that $h$ has $C^{r}$ smoothness in $x_{0}$, one may observe that for any integer $k$ such that $0 \leq k \leq r$, and for any $C^{k}$ functions $h_{1}, h_{2}$ whose derivatives up to order $k$ are bounded by one, we have,

$$
\begin{aligned}
& \left\|D_{x_{0}}^{k}\left(T h_{1}\left(x_{0}\right)-T h_{2}\left(x_{0}\right)\right)\right\|_{L^{\infty}} \\
& \quad \leq C(k, M, \mu, \varepsilon) \varepsilon\left(\left\|h_{1}-h_{2}\right\|_{L^{\infty}}+\left\|D_{x}\left(h_{1}-h_{2}\right)\right\|_{L^{\infty}}+\cdots+\left\|D_{x}^{k}\left(h_{1}-h_{2}\right)\right\|_{L^{\infty}}\right) .
\end{aligned}
$$

so that $T$ turns out to be a contraction on the space of $C^{r}$ functions whose derivatives up to order $k$ are bounded by one. 
Smoothness of $h$ in $\varepsilon$ stems from the similar kind of estimates: we use the fact that $h$

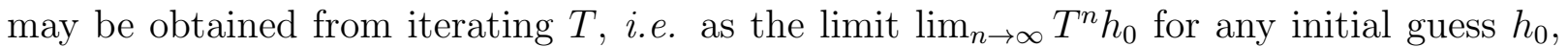
and that $\left\|D_{\varepsilon} T g\right\|_{L^{\infty}} \leq C+C \varepsilon\left\|D_{\varepsilon} g\right\|_{L^{\infty}}$ whenever $g \in B$, where $C$ is independent of $\varepsilon$, so that $\left\|D_{\varepsilon} T g\right\|_{L^{\infty}} \leq C$, to conclude that $h$ is smooth in $\varepsilon$.

Proof of point (ii).

The fact that $h$ satisfies the claimed partial differential equation merely stems from the invariance of the manifold $\{y=h(x, \varepsilon)\}$ under the forward flow of $\left(S_{\varepsilon}\right)$.

Let now $\widetilde{h}$ and $\ell$ be as in the Theorem.

Take some $K>0$ and define the set

$$
B^{\prime}=\left\{g \in B \text { such that }\|g\|_{L^{\infty}} \leq K \varepsilon^{\ell+1}\right\} .
$$

Last, for $g \in B^{\prime}$, define the mapping $S$ by

$$
S g=T(\widetilde{h}+g)-\widetilde{h} .
$$

We prove below that for $K$ large enough and $\varepsilon$ small enough, $S$ maps $B^{\prime}$ into itself. Assuming this has been proved for a while, we deduce from the previous step that $S$ is a contraction on $B^{\prime}$, as $T$ is, hence $S$ possesses a unique fixed point in $B^{\prime}$. The latter necessarily coincides with $h-\widetilde{h}$, and point (ii) is proved.

There remains to prove $S$ maps $B^{\prime}$ into itself.

To do so, and in a similar spirit as in the first step, we reformulate the mapping $S$ in an appropriate fashion. For any $g \in B^{\prime}$, we first observe the identity

$$
T(\widetilde{h}+g)\left(x_{0}\right)=\int_{-\infty}^{0} \exp \left(-\frac{s}{\varepsilon} K\right) g_{1}\left(X_{\widetilde{h}+g}(s)[\widetilde{h}+g]\left(X_{\widetilde{h}+g}(s)\right), \varepsilon\right) d s,
$$

where $X_{\widetilde{h}+g}(t) \equiv X\left(t, x_{0}, \widetilde{h}+g, \varepsilon\right)$ has been defined in (3.6). On the other hand, one may write

$$
\begin{aligned}
& -\widetilde{h}\left(x_{0}\right)=-\int_{-\infty}^{0} \frac{d}{d s}\left[\exp \left(-\frac{s}{\varepsilon} K\right) \widetilde{h}\left(X_{\widetilde{h}+g}(s)\right)\right] d s \\
& =\int_{-\infty}^{0} \exp \left(-\frac{s}{\varepsilon} K\right)\left(\frac{K}{\varepsilon} \widetilde{h}\left(X_{\widetilde{h}+g}(s)\right)-D_{x} \widetilde{h}\left(X_{\widetilde{h}+g}(s)\right) \frac{d}{d s} X_{\widetilde{h}+g}(s)\right) d s \\
& =\int_{-\infty}^{0} \exp \left(-\frac{s}{\varepsilon} K\right)\left(\frac{K}{\varepsilon} \widetilde{h}\left(X_{\widetilde{h}+g}(s)\right)-D_{x} \widetilde{h}\left(X_{\widetilde{h}+g}(s)\right) f_{0}\left(X_{\widetilde{h}+g}(s),[\widetilde{h}+g]\left(X_{\widetilde{h}+g}(s)\right), \varepsilon\right)\right) d s .
\end{aligned}
$$

Summarizing, we arrive at

$$
\begin{gathered}
(S g)\left(x_{0}\right)=\int_{-\infty}^{0} \exp \left(-\frac{s}{\varepsilon} K\right) \Phi\left(X_{\widetilde{h}+g}(s), g\left(X_{\widetilde{h}+g}(s)\right)\right) d s, \quad \text { where } \\
\begin{array}{c}
\Phi(x, g)=g_{1}(x, \widetilde{h}+g)-g_{1}(x, \widetilde{h}(x))+D_{x} \widetilde{h}(x)\left[f_{0}(x, \widetilde{h}(x))-f_{0}(x, \widetilde{h}(x)+g)\right] \\
\quad+D_{x} \widetilde{h}(x) f_{0}(x, \widetilde{h}(x))-\frac{K}{\varepsilon} \widetilde{h}(x)-g_{1}(x, \widetilde{h}(x)) .
\end{array}
\end{gathered}
$$

Now, we easily estimate

$$
\| \Phi\left(X\left(_{\widetilde{h}+g}(t), g\left(X_{\widetilde{h}+g}(t)\right)\right)\left\|_{L^{\infty}} \leq 2 M\right\| g \|_{L^{\infty}}+C_{0} \varepsilon^{\ell},\right.
$$


due to the assumed relation satisfied by $\widetilde{h}$, and where $C_{0}$ is given (it depends on the $O\left(\varepsilon^{\ell}\right)$ in the statement of the Theorem), while $M$ has the same meaning as in the previous step. Therefore, it comes

$$
\begin{aligned}
\|S g\|_{L^{\infty}} & \leq \int_{-\infty}^{0} \exp \left(+\mu \frac{s}{\varepsilon}\right)\left(2 M\|g\|_{L^{\infty}}+C_{0} \varepsilon^{\ell}\right) d s \\
& \leq 2 M \mu^{-1} \varepsilon\|g\|_{L^{\infty}}+C_{0} \mu^{-1} \varepsilon^{\ell+1} .
\end{aligned}
$$

This proves that $S$ maps $B^{\prime}$ into itself for $K$ large enough and $\varepsilon$ small enough. The proof of point (ii) is completed.

\section{Prof of point (iii)}

Unlike the previous two proofs, the proof here is constructive. We assume the formal expression $h(x, \varepsilon)=\sum_{j>1} \varepsilon^{j} h_{j}(x)$. By invariance of the central manifold, the function $h(x, \varepsilon)$ satisfies the following partial differential equation:

$$
\varepsilon D_{x} h(x, \varepsilon) f_{0}(x, h(x, \varepsilon), \varepsilon)=K h(x, \varepsilon)+\varepsilon g_{1}(x, h(x, \varepsilon), \varepsilon) .
$$

Expanding formally both sides of (3.13) around $\varepsilon=0$, we obtain

$$
\begin{aligned}
& f_{0}(x, h(x, \varepsilon), \varepsilon)=\sum_{\ell \geq 0} \varepsilon^{\ell} \overline{f_{\ell}}(x), \quad \text { where } \\
& \overline{f_{\ell}}(x):=\sum_{\substack{\alpha \geq 0, \beta \geq 0, j_{1} \geq 1, \ldots, j_{\alpha} \geq 1, j_{1}+\cdots+j_{\alpha}+\beta=\ell,}} \frac{D_{h}^{\alpha} \partial_{\varepsilon}^{\beta} f_{0}}{\alpha ! \beta !}(x, 0,0)\left(h_{j_{1}}, \ldots, h_{j_{\alpha}}\right),
\end{aligned}
$$

and similarly for $g_{1}$, so that (3.13) produces the equality

$$
\sum_{\ell \geq 2} \varepsilon^{\ell}\left(\sum_{j+k=\ell-1} D_{x} h_{j}(x) \overline{f_{k}}(x)\right)=\sum_{\ell \geq 1} \varepsilon^{\ell} K h_{\ell}(x)+\sum_{\ell \geq 1} \varepsilon^{\ell} \overline{g_{\ell-1}}(x) .
$$

Note that the factor $\overline{f_{k}}$ only depends on the $h_{j}$ 's for $1 \leq j \leq k$, and similarly for $\overline{g_{\ell-1}}$. Equating like powers of $\varepsilon$ eventually gives

$$
\begin{aligned}
& 0=K h_{1}(x)+g_{1}(x, 0,0), \\
& D_{x} h_{1}(x) f_{0}(x, 0,0)=K h_{2}(x)+D_{h} g_{1}(x, 0,0)+\partial_{\varepsilon} g_{1}(x, 0,0), \\
& \forall \ell \geq 2, \quad \sum_{j+k=\ell} D_{x} h_{j}(x) \overline{f_{k}}(x)=K h_{\ell+1}(x)+\overline{g_{\ell}}(x) .
\end{aligned}
$$

From this one deduces, using the invertibility of $K$,

$$
\begin{aligned}
& h_{1}(x)=-K^{-1} g_{1}(x, 0,0), \\
& h_{2}(x)=-K^{-1}\left(D_{h} g_{1}(x, 0,0)+\partial_{\varepsilon} g_{1}(x, 0,0)-D_{x} h_{1}(x) f_{0}(x, 0,0)\right), \\
& \forall \ell \geq 2, \quad K h_{\ell+1}(x)=-K^{-1}\left(\overline{g_{\ell}}(x)-\sum_{j+k=\ell} D_{x} h_{j}(x) \overline{f_{k}}(x)\right) .
\end{aligned}
$$

These relations allow to determine $h_{1}, h_{2}$, next $h_{\ell+1}$ as a function of $h_{1}, h_{2}, \ldots, h_{\ell}$ for any value of $\ell$. 
Now, the function $h^{[\ell]}=\varepsilon h_{1}+\cdots+\varepsilon^{\ell} h_{\ell}$ clearly satisfies the relation

$$
D_{x} h^{[\ell]}(x, \varepsilon) f_{0}\left(x, h^{[\ell]}(x, \varepsilon), \varepsilon\right)=\frac{K}{\varepsilon} h^{[\ell]}(x, \varepsilon)+g_{1}\left(x, h^{[\ell]}(x, \varepsilon), \varepsilon\right)+O\left(\varepsilon^{\ell}\right) .
$$

by construction. This information, when combined with point (ii) of the Theorem, finishes the proof of point (iii).

\section{Analysis of the discrete model}

With the notation of $(2.12)$, define the two vectors

$$
\mathbf{p}^{\varepsilon}(t):=\left(\left\{p_{i}^{\varepsilon}(t)\right\}_{i=1}^{N}\right), \quad \mathbf{q}^{\varepsilon}(t):=\left(\left\{q_{i}^{\varepsilon}(t)\right\}_{i=1}^{N}\right)
$$

as well as the two transfer operators (we use the same notation as in the continuous case (2.4) for simplicity)

$$
\begin{aligned}
& \left(\left\{p_{i}\right\}_{i=1}^{N}\right)=\mathbf{p} \in \mathbb{R}^{N} \longmapsto K_{p}(\mathbf{p}):=\left(\left\{\sum_{j=1}^{N}\left[\sigma_{p, i j} p_{j}-\sigma_{p, j i} p_{i}\right]\right\}_{i=1}^{N}\right) \in \mathbb{R}^{N}, \\
& \left(\left\{q_{i}\right\}_{i=1}^{N}\right)=\mathbf{q} \in \mathbb{R}^{N} \longmapsto K_{q}(\mathbf{q}):=\left(\left\{\sum_{j=1}^{N}\left[\sigma_{q, i j} q_{j}-\sigma_{q, j i} q_{i}\right]\right\}_{i=1}^{N}\right) \in \mathbb{R}^{N} .
\end{aligned}
$$

With this notation, the discrete system (2.12) may be written shortly

$$
\left\{\begin{array}{l}
\frac{d}{d t} \mathbf{p}^{\varepsilon}(t)=\frac{1}{\varepsilon} K_{p}\left(\mathbf{p}^{\varepsilon}(t)\right)+F_{p}\left(\mathbf{p}^{\varepsilon}(t), \mathbf{q}^{\varepsilon}(t)\right), \\
\frac{d}{d t} \mathbf{q}^{\varepsilon}(t)=\frac{1}{\varepsilon} K_{q}\left(\mathbf{q}^{\varepsilon}(t)\right)+F_{q}\left(\mathbf{p}^{\varepsilon}(t), \mathbf{q}^{\varepsilon}(t)\right), \\
\mathbf{p}^{\varepsilon}(0)=\mathbf{p}_{0}, \quad \mathbf{q}^{\varepsilon}(0)=\mathbf{q}_{0},
\end{array}\right.
$$

where $\mathbf{p}_{0}:=\left(\left\{p_{0, i}\right\}_{i=1}^{N}\right), \mathbf{q}_{0}:=\left(\left\{q_{0, i}\right\}_{i=1}^{N}\right)$, and the nonlinear functions $F_{p}(\mathbf{p}, \mathbf{q}) \in \mathbb{R}^{N}$ and $F_{q}(\mathbf{p}, \mathbf{q}) \in \mathbb{R}^{N}$ have the obvious definition, valid for any $\mathbf{p} \in \mathbb{R}^{N}$ and $\mathbf{q} \in \mathbb{R}^{N}$,

$$
F_{p}(\mathbf{p}, \mathbf{q}):=\left(\left\{a_{p, i} p_{i}-b_{p, i} p_{i} q_{i}\right\}_{i=1}^{N}\right), \quad F_{q}(\mathbf{p}, \mathbf{q}):=-\left(\left\{a_{q, i} p_{i}-b_{q, i} p_{i} q_{i}\right\}_{i=1}^{N}\right) .
$$

\subsection{Equilibrium states of the transfer operator}

Under the irreducibility assumption (2.14), we may prove the following

Theorem 4.1. (i) The kernel of the linear operator $\mathbf{p} \mapsto K_{p}(\mathbf{p})$ has dimension 1 . Besides, there is a unique equilibrium vector $\mathbf{p}_{\mathrm{eq}}=\left(\left\{p_{\mathrm{eq}, i}\right\}_{i=1}^{N}\right) \in \mathbb{R}^{N}$ such that

$$
\operatorname{ker} K_{p}=\operatorname{Vect}\left(\mathbf{p}_{\text {eq }}\right), \quad \sum_{i=1}^{N} p_{\text {eq }, i}=1, \quad \text { and } \forall i \in\{1, \ldots, N\} \quad p_{\text {eq }, i}>0 \text {. }
$$


(ii) The subspace $\mathcal{E}_{0}$ defined as

$$
\mathcal{E}_{0}:=\left\{\mathbf{x}=\left(\left\{x_{i}\right\}_{i=1}^{N}\right) \in \mathbb{R}^{N}, \text { such that } \sum_{i=1}^{N} x_{i}=0\right\} \quad\left(=\left(\left(\operatorname{ker}\left(K_{p}^{\top}\right)\right)^{\perp}\right)\right.
$$

is stable under the action of $K_{p}$. It satisfies

$$
\mathbb{R}^{N}=\operatorname{Vect}\left(\mathbf{p}_{\mathrm{eq}}\right) \oplus \mathcal{E}_{0}
$$

(iii) The restriction of $K_{p}$ to $\mathcal{E}_{0}$ has negative spectrum

$$
\sigma\left(\left.K_{p}\right|_{\mathcal{E}_{0}}\right) \subset\{z \in \mathbb{C}, \operatorname{Re}(z)<0\}
$$

In particular, there exist constants $C_{p}>0$ and $\mu_{p}>0$ such that any solution to $\frac{d}{d t} \mathbf{f}(t)=$ $K_{p}(\mathbf{f}(t))$ with initial datum $\mathbf{f}(0) \in \mathcal{E}_{0}$ satisfies

$$
\forall t \geq 0, \quad\|\mathbf{f}(t)\|_{\mathbb{R}^{N}} \leq C_{p}\|\mathbf{f}(0)\|_{\mathbb{R}^{N}} \exp \left(-\mu_{p} t\right) .
$$

(iv) Similarly, the kernel of the linear operator $\mathbf{q} \mapsto K_{q}(\mathbf{q})$ has dimension 1 , and there is a unique equilibrium vector $\mathbf{q}_{\mathrm{eq}}=\left(\left\{q_{\mathrm{eq}, i}\right\}_{i=1}^{N}\right) \in \mathbb{R}^{N}$ such that

$$
\operatorname{ker} K_{q}=\operatorname{Vect}\left(\mathbf{q}_{\mathrm{eq}}\right), \quad \sum_{i=1}^{N} q_{\mathrm{eq}, i}=1, \quad \text { and } \forall i \in\{1, \ldots, N\} \quad q_{\mathrm{eq}, i}>0 .
$$

The subspace $\mathcal{E}_{0}$ defined above is stable under the action of $K_{q}$ and satisfies

$$
\mathbb{R}^{N}=\operatorname{Vect}\left(\mathbf{q}_{\mathrm{eq}}\right) \oplus \mathcal{E}_{0} \text {. }
$$

Last, the restriction of $K_{q}$ to $\mathcal{E}_{0}$ has negative spectrum, so that there exist constants $C_{q}>0$ and $\mu_{q}>0$ such that any solution to $\frac{d}{d t} \mathbf{f}(t)=K_{q}(\mathbf{f}(t))$ with initial datum $\mathbf{f}(0) \in \mathcal{E}_{0}$ satisfies

$$
\forall t \geq 0, \quad\|\mathbf{f}(t)\|_{\mathbb{R}^{N}} \leq C_{q}\|\mathbf{f}(0)\|_{\mathbb{R}^{N}} \exp \left(-\mu_{q} t\right)
$$

Remark. The unique vector $\mathbf{p}_{\text {eq }}\left(\right.$ resp. $\mathbf{q}_{\text {eq }}$ ) describes the equilibrium repartition of preys (resp. predators) along the pure migration dynamics,

$$
\frac{d}{d t} \mathbf{p}(t)=K_{p}(\mathbf{p}(t))
$$

In particular, and as a consequence of the above theorem, any solution to (4.6) satisfies

$$
\mathbf{p}(t) \underset{t \rightarrow+\infty}{\longrightarrow}\left(\sum_{i=1}^{N} p_{i}(0)\right) \mathbf{p}_{\mathrm{eq}}
$$


where the prefactor $\sum_{i=1}^{N} p_{i}(0)$ simply is the total number of preys a the initial time (recall this number is constant along the dynamics of (4.6)). The rate of convergence in (4.7) is exponential. Note in passing that $p_{\mathrm{eq}, i}>0$ for all $i$ : at equilibrium, all sites are occupied by a positive proportion of preys. This is a direct consequence of the irreducibility assumption (2.14).

Remark. Note that the same subspace $\mathcal{E}_{0}$ supplements both the kernel of $K_{p}$ and that of $K_{q}$. This is due to the fact that both $K_{p}$ and $K_{q}$ are conservative: $\mathcal{E}_{0}$ is the subspace orthogonal to vector $\mathbf{1}=(1, \ldots, 1)^{\top}$.

\section{Proof of Theorem 4.1.}

The proof is essentially based on the Perron-Frobenius theorem, see e.g. [Se].

As $K_{p}$ and $K_{q}$ are irreducible, it turns out that 0 is a simple eigenvalue of both operators, and both possess a unique associated eigenvector with positive entries that sum up to unity. Besides, all other eigenvalues of both operator have negative real part. The fact that $\mathcal{E}_{0}$ is stable upon the action of $K_{p}$ and $K_{q}$ is a direct consequence of the fact that both operators are conservative. The existence of the exponential rates of convergence $\mu_{p}$ and $\mu_{q}$ now come from the fact that the system under study is finite dimensional. This proves the Theorem.

\subsection{Writing the discrete model in slow-fast form}

Theorem 4.1 allows to identify the "good" slow and fast variables in the original model (2.12), or equivalently (4.4). Indeed, associated with the decompositions

$$
\mathbb{R}^{N}=\operatorname{Vect}\left(\mathbf{p}_{\text {eq }}\right) \oplus \mathcal{E}_{0} \quad \text { and } \quad \mathbb{R}^{N}=\operatorname{Vect}\left(\mathbf{q}_{\text {eq }}\right) \oplus \mathcal{E}_{0},
$$

one may split the solution $\left(\mathbf{p}^{\varepsilon}(t), \mathbf{q}^{\varepsilon}(t)\right)$ to $(2.12)$ or (4.4) into

$$
\begin{aligned}
& \mathbf{p}^{\varepsilon}(t)=P_{\mathrm{d}}^{\varepsilon}(t) \mathbf{p}_{\mathrm{eq}}+\mathbf{R}_{p}^{\varepsilon}(t) \in \operatorname{Vect}\left(\mathrm{p}_{\mathrm{eq}}\right) \oplus \mathcal{E}_{0}, \\
& \mathbf{q}^{\varepsilon}(t)=Q_{\mathrm{d}}^{\varepsilon}(t) \mathbf{q}_{\mathrm{eq}}+\mathbf{R}_{q}^{\varepsilon}(t) \in \operatorname{Vect}\left(\mathrm{q}_{\mathrm{eq}}\right) \oplus \mathcal{E}_{0},
\end{aligned}
$$

where $P_{\mathrm{d}}^{\varepsilon}(t), \mathbf{R}_{p}^{\varepsilon}(t), Q_{\mathrm{d}}^{\varepsilon}(t), \mathbf{R}_{q}^{\varepsilon}(t)$ have the obvious values (in accordance with (2.15))

$$
\begin{array}{ll}
P_{\mathrm{d}}^{\varepsilon}(t)=\sum_{i=1}^{N} p_{i}^{\varepsilon}(t)=: S \mathbf{p}^{\varepsilon}(t), & \mathbf{R}_{p}^{\varepsilon}(t)=\mathbf{p}^{\varepsilon}(t)-P_{\mathrm{d}}^{\varepsilon}(t) \mathbf{p}_{\mathrm{eq}}=: R_{p} \mathbf{p}^{\varepsilon}(t), \\
Q_{\mathrm{d}}^{\varepsilon}(t)=\sum_{i=1}^{N} q_{i}^{\varepsilon}(t)=: S \mathbf{q}^{\varepsilon}(t), & \mathbf{R}_{q}^{\varepsilon}(t)=\mathbf{q}^{\varepsilon}(t)-Q_{\mathrm{d}}^{\varepsilon}(t) \mathbf{q}_{\mathrm{eq}}=: R_{q} \mathbf{q}^{\varepsilon}(t),
\end{array}
$$

and the above equation implicitely defines the notation, valid for any $\mathbf{p}=\left(\left\{p_{i}\right\}_{i=1}^{N}\right) \in \mathbb{R}^{N}$,

$$
S \mathbf{p}:=\sum_{i=1}^{N} p_{i}, \quad R_{p} \mathbf{p}:=\mathbf{p}-(S \mathbf{p}) \mathbf{p}_{\mathrm{eq}}, \quad R_{q} \mathbf{q}:=\mathbf{q}-(S \mathbf{q}) \mathbf{q}_{\mathrm{eq}} .
$$

Naturally, the two changes of unknowns $\mathbf{p}^{\varepsilon}(t) \in \mathbb{R}^{N} \mapsto\left(P_{\mathrm{d}}^{\varepsilon}(t), \mathbf{R}_{p}^{\varepsilon}(t)\right) \in \mathbb{R} \times \mathcal{E}_{0}$ and $\mathbf{q}^{\varepsilon}(t) \in \mathbb{R}^{N} \mapsto\left(Q_{\mathrm{d}}^{\varepsilon}(t), \mathbf{R}_{q}^{\varepsilon}(t)\right) \in \mathbb{R} \times \mathcal{E}_{0}$ are one-to-one. The explicit value of the equilibrium 
vectors $\mathbf{p}_{\mathrm{eq}}$ and $\mathbf{q}_{\mathrm{eq}}$ cannot be given in any general form: it has to be computed for each specific model independently. As a consequence, the changes of unknowns $\mathbf{p}^{\varepsilon}(t) \in \mathbb{R}^{N} \mapsto$ $\left(P_{\mathrm{d}}^{\varepsilon}(t), \mathbf{R}_{p}^{\varepsilon}(t)\right) \in \mathbb{R} \times \mathcal{E}_{0}$ and $\mathbf{q}^{\varepsilon}(t) \in \mathbb{R}^{N} \mapsto\left(Q_{\mathrm{d}}^{\varepsilon}(t), \mathbf{R}_{q}^{\varepsilon}(t)\right) \in \mathbb{R} \times \mathcal{E}_{0}$ cannot be made explicit in any general way neither: they need to be made explicit for each specific situation independently.

With these new variables at hand, the original system (2.12) anyhow becomes

$$
\left\{\begin{array}{l}
\frac{d}{d t} P_{\mathrm{d}}^{\varepsilon}(t)=S F_{p}\left(P_{\mathrm{d}}^{\varepsilon}(t) \mathbf{p}_{\mathrm{eq}}+\mathbf{R}_{p}^{\varepsilon}(t), Q_{\mathrm{d}}^{\varepsilon}(t) \mathbf{q}_{\mathrm{eq}}+\mathbf{R}_{q}^{\varepsilon}(t)\right) \\
\frac{d}{d t} Q_{\mathrm{d}}^{\varepsilon}(t)=S F_{q}\left(P_{\mathrm{d}}^{\varepsilon}(t) \mathbf{p}_{\mathrm{eq}}+\mathbf{R}_{p}^{\varepsilon}(t), Q_{\mathrm{d}}^{\varepsilon}(t) \mathbf{q}_{\mathrm{eq}}+\mathbf{R}_{q}^{\varepsilon}(t)\right) \\
\frac{d}{d t} \mathbf{R}_{p}^{\varepsilon}(t)=\frac{1}{\varepsilon} K_{p} \mathbf{R}_{p}^{\varepsilon}(t)+R_{p} F_{p}\left(P_{\mathrm{d}}^{\varepsilon}(t) \mathbf{p}_{\mathrm{eq}}+\mathbf{R}_{p}^{\varepsilon}(t), Q_{\mathrm{d}}^{\varepsilon}(t) \mathbf{q}_{\mathrm{eq}}+\mathbf{R}_{q}^{\varepsilon}(t)\right) \\
\frac{d}{d t} \mathbf{R}_{q}^{\varepsilon}(t)=\frac{1}{\varepsilon} K_{q} \mathbf{R}_{q}^{\varepsilon}(t)+R_{q} F_{q}\left(P_{\mathrm{d}}^{\varepsilon}(t) \mathbf{p}_{\mathrm{eq}}+\mathbf{R}_{p}^{\varepsilon}(t), Q_{\mathrm{d}}^{\varepsilon}(t) \mathbf{q}_{\mathrm{eq}}+\mathbf{R}_{q}^{\varepsilon}(t)\right) \\
P_{\mathrm{d}}^{\varepsilon}(0)=S \mathbf{p}_{0}, \quad Q_{\mathrm{d}}^{\varepsilon}(0)=S \mathbf{q}_{0}, \quad \mathbf{R}_{p}^{\varepsilon}(0)=R_{p} \mathbf{p}_{0}, \quad \mathbf{R}_{q}^{\varepsilon}(0)=R_{q} \mathbf{q}_{0}
\end{array}\right.
$$

where we use the notation (4.9) and the nonlinear functions $F_{p}$ and $F_{q}$ (the Lotka-Volterra part of the population dynamics under study) have been defined in (4.5).

System (4.11) now is in the necessary slow-fast form required to apply the central manifold theorem. Indeed, setting ${ }^{3}$

$$
X_{\mathrm{d}}^{\varepsilon}(t)=\left(P_{\mathrm{d}}^{\varepsilon}(t), Q_{\mathrm{d}}^{\varepsilon}(t)\right) \in \mathbb{R}^{2}, \text { and } Y_{\mathrm{d}}^{\varepsilon}(t)=\left(\mathbf{R}_{p}^{\varepsilon}(t), \mathbf{R}_{q}^{\varepsilon}(t)\right) \in \mathcal{E}_{0} \times \mathcal{E}_{0} .
$$

together with

$$
\begin{aligned}
& f_{0, \mathrm{~d}}\left(X_{\mathrm{d}}^{\varepsilon}, Y_{\mathrm{d}}^{\varepsilon}\right)=\left(\begin{array}{c}
S F_{p}\left(P^{\varepsilon}(t) \mathbf{p}_{\mathrm{eq}}+\mathbf{R}_{p}^{\varepsilon}(t), Q^{\varepsilon}(t) \mathbf{q}_{\mathrm{eq}}+\mathbf{R}_{q}^{\varepsilon}(t)\right) \\
S F_{q}\left(P^{\varepsilon}(t) \mathbf{p}_{\mathrm{eq}}+\mathbf{R}_{p}^{\varepsilon}(t), Q^{\varepsilon}(t) \mathbf{q}_{\mathrm{eq}}+\mathbf{R}_{q}^{\varepsilon}(t)\right)
\end{array}\right), \\
& K Y_{\mathrm{d}}^{\varepsilon}=\left(\begin{array}{c}
K_{p}\left(\mathbf{R}_{p}^{\varepsilon}\right)(t) \\
K_{q}\left(\mathbf{R}_{q}^{\varepsilon}\right)(t)
\end{array}\right) \\
& g_{1, \mathrm{~d}}\left(X_{\mathrm{d}}^{\varepsilon}, Y_{\mathrm{d}}^{\varepsilon}\right)=\left(\begin{array}{c}
R_{p} F_{p}\left(P^{\varepsilon}(t) \mathbf{p}_{\mathrm{eq}}+\mathbf{R}_{p}^{\varepsilon}(t), Q^{\varepsilon}(t) \mathbf{q}_{\mathrm{eq}}+\mathbf{R}_{q}^{\varepsilon}(t)\right) \\
R_{q} F_{q}\left(P^{\varepsilon}(t) \mathbf{p}_{\mathrm{eq}}+\mathbf{R}_{p}^{\varepsilon}(t), Q^{\varepsilon}(t) \mathbf{q}_{\mathrm{eq}}+\mathbf{R}_{q}^{\varepsilon}(t)\right)
\end{array}\right),
\end{aligned}
$$

system (4.11) takes the form

$$
\left(S_{\varepsilon, \mathrm{d}}\right)\left\{\begin{array}{lrl}
\frac{d}{d t} X_{\mathrm{d}}^{\varepsilon}(t)=f_{0, \mathrm{~d}}\left(X_{\mathrm{d}}^{\varepsilon}(t), Y_{\mathrm{d}}^{\varepsilon}(t), \varepsilon\right), & X_{\mathrm{d}}^{\varepsilon}(0)=x_{0} \\
\frac{d}{d t} Y_{\mathrm{d}}^{\varepsilon}(t)=\frac{K}{\varepsilon} Y_{\mathrm{d}}^{\varepsilon}(t)+g_{1, \mathrm{~d}}\left(X_{\mathrm{d}}^{\varepsilon}(t), Y_{\mathrm{d}}^{\varepsilon}(t), \varepsilon\right), & Y_{\mathrm{d}}^{\varepsilon}(0)=y_{0}
\end{array}\right.
$$

\subsection{Application of the central manifold Theorem}

When written in the form (4.11) or equivalently $\left(S_{\varepsilon, \mathrm{d}}\right)$, the discrete system (2.12) clearly satisfies the assumptions of the central manifold Theorem 3.1, with $E=\mathbb{R} \times \mathbb{R}, F=\mathcal{E}_{0} \times \mathcal{E}_{0}$, and $r$ may be any integer (the functions at hand actually have $C^{\infty}$ smoothness). Strictly speaking, the relevant functions $f_{0, \mathrm{~d}}$ and $g_{1, \mathrm{~d}}$ involved in $\left(S_{\varepsilon, \mathrm{d}}\right)$ or equivalently (4.11) are

\footnotetext{
${ }^{3}$ The lower index "d" again refers to the discrete system
} 
not bounded over the whole of $E \times F$, yet this is a harmless point: for any given time $T$, the unknowns $X_{\mathrm{d}}^{\varepsilon}(t)=\left(P_{\mathrm{d}}^{\varepsilon}(t), Q_{\mathrm{d}}^{\varepsilon}(t)\right)$ and $Y_{\mathrm{d}}^{\varepsilon}(t)=\left(\mathbf{R}_{p}^{\varepsilon}(t), \mathbf{R}_{q}^{\varepsilon}(t)\right)$ anyhow take bounded values, hence $f_{0, \mathrm{~d}}$ and $g_{1, \mathrm{~d}}$ may anyhow be truncated accordingly without altering the values of $\left(X_{\mathrm{d}}^{\varepsilon}(t), Y_{\mathrm{d}}^{\varepsilon}(t)\right)$ on $[0, T]$. Recall that both linear operators $K_{p}$ and $K_{q}$ are bijections from $\mathcal{E}_{0}$ to itself, so that $K$ in (4.14) is a bijection from $\mathcal{E}_{0} \times \mathcal{E}_{0}$ to itself. Recall also that the Perron-Frobenius theorem (see Theorem 4.1) asserts the existence of a $\mu>0$ and $C>0$ such that

$$
\forall Y \in \mathcal{E}_{0} \times \mathcal{E}_{0}, \quad \forall t \geq 0, \quad \forall \varepsilon>0, \quad\left|\exp \left(K \frac{t}{\varepsilon}\right) Y\right| \leq C|Y| \exp \left(-\mu \frac{t}{\varepsilon}\right),
$$

where |.| stand for the usual euclidean norm over $\mathbb{R}^{2 N}$.

The central manifold theorem now asserts the existence of

$$
h_{\mathrm{d}}(X, \varepsilon)=\varepsilon h_{1, \mathrm{~d}}(X)+\varepsilon^{2} h_{2, \mathrm{~d}}(X)+\cdots
$$

such that Theorem 3.1 holds true when applied to the discrete system (4.11) or equivalently $\left(S_{\varepsilon, \mathrm{d}}\right)$. Associated with the function $h_{\mathrm{d}}(X, \varepsilon)$, one may write the reduced dynamics

$$
\left(S_{\varepsilon, \mathrm{d}}^{[\infty]}\right) \quad \frac{d}{d t} X_{\mathrm{d}}^{\varepsilon,[\infty]}(t)=f_{0, \mathrm{~d}}\left(X_{\mathrm{d}}^{\varepsilon,[\infty]}(t), h\left(X_{\mathrm{d}}^{\varepsilon,[\infty]}(t), \varepsilon\right), \varepsilon\right), \quad Y_{\mathrm{d}}^{\varepsilon,[\infty]}(0)=h_{\mathrm{d}}\left(X_{\mathrm{d}}^{\varepsilon,[\infty]}(t), \varepsilon\right)
$$

as well as, for any integer $\ell$, the truncated versions of $\left(S_{\varepsilon, \mathrm{d}}^{[\infty]}\right)$, namely

$$
\left(S_{\varepsilon, \mathrm{d}}^{[\ell]}\right) \quad \frac{d}{d t} X_{\mathrm{d}}^{\varepsilon,[\ell]}(t)=f_{0, \mathrm{~d}}\left(X_{\mathrm{d}}^{\varepsilon,[\ell]}(t), h^{[\ell]}\left(X_{\mathrm{d}}^{\varepsilon,[\ell]}(t), \varepsilon\right), \varepsilon\right), \quad Y_{\mathrm{d}}^{\varepsilon,[\ell]}(0)=h_{\mathrm{d}}\left(X_{\mathrm{d}}^{\varepsilon,[\ell]}(t), \varepsilon\right),
$$

as we did in the previous paragraph.

To complete our analysis we now compute the functions $h_{1, \mathrm{~d}}, h_{2, \mathrm{~d}}$, etc., and study the properties of the reduced models $\left(S_{\varepsilon, \mathrm{d}}^{[\infty]}\right)$ and $\left(S_{\varepsilon, \mathrm{d}}^{[\ell]}\right)$.

\subsubsection{Computing the reduced models}

To compute the actual values of $h_{1, \mathrm{~d}}, h_{2, \mathrm{~d}}$, etc. we argue as in the proof of Theorem 3.1 point (iii). We first write

$$
\frac{D h_{\mathrm{d}}(X, \varepsilon)}{D X} f_{0, \mathrm{~d}}(X, h(X, \varepsilon))=\frac{1}{\varepsilon} K h_{\mathrm{d}}(X, \varepsilon)+g_{1, \mathrm{~d}}\left(X, h_{\mathrm{d}}(X, \varepsilon)\right) .
$$

Next expanding $h_{\mathrm{d}}=\varepsilon h_{\mathrm{d}, 1}+\varepsilon^{2} h_{2, \mathrm{~d}}+\cdots$, and using the fact that $f_{0, \mathrm{~d}}$ and $g_{1, \mathrm{~d}}$ are at most quadratic in $Y$ (see (4.13) and (4.15)) gives

$$
\begin{aligned}
& {\left[\varepsilon D_{X} h_{1, \mathrm{~d}}(X)+\varepsilon^{2} D_{X} h_{2, \mathrm{~d}}(X)+\cdots\right] \times} \\
& {\left[f_{0, \mathrm{~d}}(X, 0)+D_{Y} f_{0, \mathrm{~d}}(X, 0)\left(\varepsilon h_{1, \mathrm{~d}}(X)+\varepsilon^{2} h_{2, \mathrm{~d}}(X)+\cdots\right)\right.} \\
& \left.\quad+\frac{1}{2} D_{Y, Y}^{2} f_{0, \mathrm{~d}}(X, 0)\left(\varepsilon h_{1, \mathrm{~d}}(X)+\varepsilon^{2} h_{2, \mathrm{~d}}(X)+\cdots\right)\left(\varepsilon h_{1, \mathrm{~d}}(X)+\varepsilon^{2} h_{2, \mathrm{~d}}(X)+\cdots\right)\right] \\
& =K\left[h_{1, \mathrm{~d}}(X)+\varepsilon h_{2, \mathrm{~d}}(X)+\cdots\right]+g_{1, \mathrm{~d}}(X, 0)+D_{Y} g_{1, \mathrm{~d}}(X, 0)\left(\varepsilon h_{1, \mathrm{~d}}(X)+\varepsilon^{2} h_{2, \mathrm{~d}}(X)+\cdots\right) \\
& \quad+\frac{1}{2} D_{Y, Y}^{2} g_{1, \mathrm{~d}}(X, 0)\left(\varepsilon h_{1, \mathrm{~d}}(X)+\varepsilon^{2} h_{2, \mathrm{~d}}(X)+\cdots\right)\left(\varepsilon h_{1, \mathrm{~d}}(X)+\varepsilon^{2} h_{2, \mathrm{~d}}(X)+\cdots\right),
\end{aligned}
$$


where $D_{Y}$ and $D_{Y, Y}^{2}$ are short-hand notations for $D / D Y$ and $D^{2} / D Y D Y$. Identifying like powers of $\varepsilon$, eventually gives

$$
\begin{aligned}
& 0=K h_{1, \mathrm{~d}}(X)+g_{1, \mathrm{~d}}(X, 0) \\
& D_{X} h_{1, \mathrm{~d}}(X) f_{0, \mathrm{~d}}(X, 0)=K h_{2, \mathrm{~d}}(X)+D_{Y} g_{1, \mathrm{~d}}(X, 0) h_{1, \mathrm{~d}}(X) \\
& D_{X} h_{2, \mathrm{~d}}(X) f_{0, \mathrm{~d}}(X, 0)+D_{X} h_{1, \mathrm{~d}}(X) D_{Y} f_{0, \mathrm{~d}}(X, 0) h_{1, \mathrm{~d}}(X)+\frac{1}{2} D_{Y, Y}^{2} f_{0, \mathrm{~d}}(X, 0) h_{1, \mathrm{~d}}(X) h_{1, \mathrm{~d}}(X) \\
& \quad=K h_{3, \mathrm{~d}}(X)+D_{Y} g_{1, \mathrm{~d}}(X, 0) h_{2, \mathrm{~d}}(X)+\frac{1}{2} D_{Y, Y}^{2} g_{1, \mathrm{~d}}(X, 0) h_{1, \mathrm{~d}}(X) h_{1, \mathrm{~d}}(X),
\end{aligned}
$$

and so on. This gives the explicit values

$$
\begin{aligned}
h_{1, \mathrm{~d}}(X)= & -K^{-1} g_{1, \mathrm{~d}}(X, 0), \\
h_{2, \mathrm{~d}}(X)= & K^{-1}\left[D_{X} h_{1, \mathrm{~d}}(X) f_{0, \mathrm{~d}}(X, 0)-D_{Y} g_{1, \mathrm{~d}}(X, 0) h_{1, \mathrm{~d}}(X)\right], \\
h_{3, \mathrm{~d}}(X)= & K^{-1}\left[D_{X} h_{2, \mathrm{~d}}(X) f_{0, \mathrm{~d}}(X, 0)+D_{X} h_{1, \mathrm{~d}}(X) D_{Y} f_{0, \mathrm{~d}}(X, 0) h_{1, \mathrm{~d}}(X)\right. \\
& +\frac{1}{2} D_{Y, Y}^{2} f_{0, \mathrm{~d}}(X, 0) h_{1, \mathrm{~d}}(X) h_{1, \mathrm{~d}}(X)-D_{Y} g_{1, \mathrm{~d}}(X, 0) h_{2, \mathrm{~d}}(X) \\
& \left.-\frac{1}{2} D_{Y, Y}^{2} g_{1, \mathrm{~d}}(X, 0) h_{1, \mathrm{~d}}(X) h_{1, \mathrm{~d}}(X)\right]
\end{aligned}
$$

and so on, where $K^{-1}$ is the inverse of $K$ as an operator on $\mathcal{E}_{0} \times \mathcal{E}_{0}$. The reduced models $\left(S_{\varepsilon, \mathrm{d}}^{[\ell]}\right)$ may now be explicitely written down, for any value of $\ell$. For definiteness we only give here the explicit values for the values $\ell=0,1$, as based on the previous explicit computations.

Theorem 4.2. Define the vectors of prey-predation coefficients

$$
\mathbf{a}_{p}:=\left(\left\{a_{p, i}\right\}_{i=1}^{N}\right), \quad \mathbf{b}_{p}:=\left(\left\{b_{p, i}\right\}_{i=1}^{N}\right), \quad \mathbf{a}_{q}:=\left(\left\{a_{q, i}\right\}_{i=1}^{N}\right), \quad \mathbf{b}_{q}:=\left(\left\{b_{q, i}\right\}_{i=1}^{N}\right) .
$$

For any vectors $\mathbf{u}, \mathbf{v}$ and $\mathbf{w}$ belonging to $\mathbb{R}^{N}$, denote

$$
\mathbf{u} \mathbf{v}:=\left(\left\{u_{i} v_{i}\right\}_{i=1}^{N}\right), \text { and similarly } \mathbf{u} \mathbf{v} \mathbf{w}:=\left(\left\{u_{i} v_{i} w_{i}\right\}_{i=1}^{N}\right) .
$$

Then, using the operators $S, R_{p}$ and $R_{q}$ defined in (4.10), we have,

(i) the zeroth order reduced dynamics $\left(S_{\varepsilon, \mathrm{d}}^{[0]}\right)$ provided by the central manifold Theorem has the explicit value

$$
\left(S_{\varepsilon, \mathrm{d}}^{[0]}\right) \quad\left\{\begin{aligned}
\frac{d}{d t} P_{\mathrm{d}}^{\varepsilon,[0]}(t) & =A_{p}^{0} P_{\mathrm{d}}^{\varepsilon,[0]}(t)-B_{p}^{0} P_{\mathrm{d}}^{\varepsilon,[0]}(t) Q_{\mathrm{d}}^{\varepsilon,[0]}(t), \\
\frac{d}{d t} Q_{\mathrm{d}}^{\varepsilon,[0]}(t) & =-A_{q}^{0} Q_{\mathrm{d}}^{\varepsilon,[0]}(t)+B_{q}^{0} P_{\mathrm{d}}^{\varepsilon,[0]}(t) Q_{\mathrm{d}}^{\varepsilon,[0]}(t),
\end{aligned}\right.
$$

where the positive coefficients $A_{p}^{0}, B_{p}^{0}, A_{q}^{0}, B_{q}^{0}$, have the values

$$
A_{p}^{0}=S\left(\mathbf{a}_{p} \mathbf{p}_{\mathrm{eq}}\right), \quad B_{p}^{0}=S\left(\mathbf{b}_{p} \mathbf{p}_{\mathrm{eq}} \mathbf{q}_{\mathrm{eq}}\right), \quad A_{q}^{0}=S\left(\mathbf{a}_{q} \mathbf{q}_{\mathrm{eq}}\right), \quad B_{q}^{0}=S\left(\mathbf{b}_{q} \mathbf{p}_{\mathrm{eq}} \mathbf{q}_{\mathrm{eq}}\right) .
$$

(ii) the first order reduced dynamics $\left(S_{\varepsilon, \mathrm{d}}^{[1]}\right)$ provided by the central manifold Theorem has 
the explicit value

$$
\left(S_{\varepsilon, \mathrm{d}}^{[1]}\right)\left\{\begin{aligned}
\frac{d}{d t} P_{\mathrm{d}}^{\varepsilon,[1]}(t)= & \left(A_{p}^{0}+\varepsilon A_{p}^{1}\right) P_{\mathrm{d}}^{\varepsilon,[1]}(t)-\left(B_{p}^{0}+\varepsilon B_{p}^{1}\right) P_{\mathrm{d}}^{\varepsilon,[1]}(t) Q_{\mathrm{d}}^{\varepsilon,[1]}(t) \\
& +\varepsilon C_{p}^{1}\left(P_{\mathrm{d}}^{\varepsilon,[1]}(t)\right)^{2} Q_{\mathrm{d}}^{\varepsilon,[1]}(t)+\varepsilon D_{p}^{1} P_{\mathrm{d}}^{\varepsilon,[1]}(t)\left(Q_{\mathrm{d}}^{\varepsilon,[1]}(t)\right)^{2} \\
\frac{d}{d t} Q_{\mathrm{d}}^{\varepsilon,[1]}(t)= & -\left(A_{q}^{0}+\varepsilon A_{q}^{1}\right) Q_{\mathrm{d}}^{\varepsilon,[1]}(t)+\left(B_{q}^{0}+\varepsilon B_{q}^{1}\right) P_{\mathrm{d}}^{\varepsilon,[1]}(t) Q_{\mathrm{d}}^{\varepsilon,[1]}(t) \\
& +\varepsilon C_{q}^{1}\left(P_{\mathrm{d}}^{\varepsilon,[1]}(t)\right)^{2} Q_{\mathrm{d}}^{\varepsilon,[1]}(t)+\varepsilon D_{q}^{1} P_{\mathrm{d}}^{\varepsilon,[1]}(t)\left(Q_{\mathrm{d}}^{\varepsilon,[1]}(t)\right)^{2}
\end{aligned}\right.
$$

where the positive coefficients $A_{p}^{0}, B_{p}^{0}, A_{q}^{0}, B_{q}^{0}$ are defined before while

$$
\begin{aligned}
& A_{p}^{1}=S\left(\mathbf{a}_{p} \widetilde{\mathbf{a}_{p}}\right), \quad A_{q}^{1}=S\left(\mathbf{a}_{q} \widetilde{\mathbf{a}_{q}}\right), \\
& B_{p}^{1}=S\left(-\mathbf{a}_{p} \widetilde{\mathbf{b}_{p}}+\mathbf{b}_{p} \widetilde{\mathbf{a}_{q}} \mathbf{p}_{\mathrm{eq}}+\mathbf{b}_{p} \widetilde{\mathbf{a}_{p}} \mathbf{q}_{\mathrm{eq}}\right), \\
& B_{q}^{1}=S\left(-\mathbf{a}_{q} \widetilde{\mathbf{b}_{q}}+\mathbf{b}_{q} \widetilde{\mathbf{a}_{p}} \mathbf{q}_{\mathrm{eq}}+\mathbf{b}_{q} \widetilde{\mathbf{a}_{q}} \mathbf{p}_{\mathrm{eq}}\right), \\
& C_{p}^{1}=-S\left(\mathbf{b}_{p} \widetilde{\mathbf{b}_{q}} \mathbf{p}_{\mathrm{eq}}\right), \quad D_{p}^{1}=-S\left(\mathbf{b}_{p} \widetilde{\mathbf{b}_{p}} \mathbf{q}_{\mathrm{eq}}\right), \\
& C_{q}^{1}=+S\left(\mathbf{b}_{q} \widetilde{\mathbf{b}_{q}} \mathbf{p}_{\mathrm{eq}}\right), \quad D_{q}^{1}=+S\left(\mathbf{b}_{q} \widetilde{\mathbf{b}_{p}} \mathbf{q}_{\mathrm{eq}}\right),
\end{aligned}
$$

and we define

$$
\begin{array}{ll}
\widetilde{\mathbf{a}_{p}}:=-K_{p}^{-1} R_{p}\left(\mathbf{a}_{p} \mathbf{p}_{\mathrm{eq}}\right), & \widetilde{\mathbf{a}_{q}}:=+K_{q}^{-1} R_{q}\left(\mathbf{a}_{q} \mathbf{q}_{\mathrm{eq}}\right), \\
\widetilde{\mathbf{b}_{p}}:=+K_{p}^{-1} R_{p}\left(\mathbf{b}_{p} \mathbf{p}_{\mathrm{eq}} \mathbf{q}_{\mathrm{eq}}\right), & \widetilde{\mathbf{b}_{q}}:=-K_{q}^{-1} R_{q}\left(\mathbf{b}_{q} \mathbf{p}_{\mathrm{eq}} \mathbf{q}_{\mathrm{eq}}\right),
\end{array}
$$

(iii) similarly, for any integer $\ell$, the $\ell$-th order reduced model $\left(S_{\varepsilon, \mathrm{d}}^{[\ell]}\right)$ provided by the central manifold Theorem is a differential system of Lotka-Volterra type with corrective terms of order $\varepsilon, \varepsilon^{2}$, and so on up to $\varepsilon^{\ell}$ which are polynomial of degree 3,4 , and so on up to $\ell+1$ in the prey and predator variables.

Remark. The zeroth order reduced system $\left(S_{\varepsilon, \mathrm{d}}^{[0]}\right)$ is a standard Lotka-Volterra system for the total number of preys resp. predators. Needless to say, the coefficients $A_{p}^{0}, B_{p}^{0}, A_{q}^{0}, B_{q}^{0}$ of the reduced population dynamics are appropriate averages of the original coefficients $\mathbf{a}_{p}$, $\mathbf{b}_{p}, \mathbf{a}_{q}, \mathbf{b}_{q}$.

Note however that, being a Lotka-Volterra system, eq. $\left(S_{\varepsilon, \mathrm{d}}^{[0]}\right)$ possesses an equilibrium state, also called center, which is naturally unstable: cycles develop in the vicinity of the equilibrium state. Therefore, since the reduced model $\left(S_{\varepsilon, \mathrm{d}}^{[0]}\right)$ only is a zeroth order approximation of the true original dynamics $\left(S_{\varepsilon, \mathrm{d}}\right)$ or equivalently $(2.12)$ (see Theorem e.g. 4.3 below), the higher order corrections $\left(S_{\varepsilon, \mathrm{d}}^{[\ell]}\right)(\ell \geq 1)$ clearly give a crucial information about whether the true dynamics $\left(S_{\varepsilon, \mathrm{d}}\right)$ has cycles (as does its zeroth order approximation), or not.

In other words, the point in developping the central manifold to higher order not only is to obtain higher order approximations of the original populations dynamics $\left(S_{\varepsilon, \mathrm{d}}\right)$ or (2.12): it also gives a qualitative information about the behaviour of the original dynamics in the vicinity of the center, namely it asserts whether the cycles persist when $\varepsilon>0$ is 
small but definitely nonzero, and, in the generic case where the cycles do not persist, it also gives information about whether the center is attractive or repulsive when $\varepsilon>0$. This observation is mainly due to Poggiale in [Po1]. We refer to the discussion performed in section 6 on that point.

\section{Proof of Theorem 4.2.}

It is an easy consequence of formulae (4.17) together with (4.13), (4.14), (4.15).

\subsubsection{Error estimates between the original model and the reduced models}

The above Theorem 4.2 is naturally completed with the following error estimates.

Theorem 4.3. Take a fixed time $T>0$. Take a given number $0<\mu^{\prime}<\mu$, where $\mu$ is as in (4.16). Take an initial datum $x_{0} \in \mathbb{R}^{2}$ and $y_{0} \in \mathcal{E}_{0}^{2}$. Then the following properties hold true :

(i) there exists an initial datum $x_{0}^{\varepsilon}$ such that the solution $X_{\mathrm{d}}^{\varepsilon,[\infty]}(t)$ to the above defined reduced dynamics $\left(S_{\varepsilon, \mathrm{d}}^{[\infty]}\right)$ with initial datum

$$
X_{\mathrm{d}}^{\varepsilon,[\infty]}(0)=x_{0}^{\varepsilon}
$$

satisfies the exponential decay estimate, valid for any $t \in[0, T]$,

$$
\left|X_{\mathrm{d}}^{\varepsilon}(t)-X_{\mathrm{d}}^{\varepsilon,[\infty]}(t)\right|+\left|Y_{\mathrm{d}}^{\varepsilon}(t)-h_{\mathrm{d}}\left(X_{\mathrm{d}}^{\varepsilon,[\infty]}(t), \varepsilon\right)\right| \leq C_{T} \exp \left(-\frac{\mu^{\prime}}{\varepsilon} t\right)
$$

for some constant $C_{T}>0$, independent of $\varepsilon$.

(ii) besides, taking the same initial datum $x_{0}^{\varepsilon}$ as in the previous point, the solution $X_{\mathrm{d}}^{\varepsilon,[\ell]}(t)$ to the above defined reduced dynamics $\left(S_{\varepsilon, \mathrm{d}}^{[\ell]}\right)$ with initial datum

$$
X_{\mathrm{d}}^{\varepsilon,[\ell]}(0)=x_{0}^{\varepsilon}
$$

satisfies the polynomial decay estimate, valid for any $t \in[0, T]$,

$$
\left|X_{\mathrm{d}}^{\varepsilon}(t)-X_{\mathrm{d}}^{\varepsilon,[\ell]}(t)\right|+\left|Y_{\mathrm{d}}^{\varepsilon}(t)-h_{\mathrm{d}}\left(X_{\mathrm{d}}^{\varepsilon,[\infty]}(t), \varepsilon\right)\right| \leq C_{T} \varepsilon^{\ell+1}+C_{T} \exp \left(-\frac{\mu^{\prime}}{\varepsilon} t\right),
$$

for some constant $C_{T}>0$, independent of $\varepsilon$.

(iii) In particular, writing

$$
X_{\mathrm{d}}^{\varepsilon,[\infty]}(t)=\left(P_{\mathrm{d}}^{\varepsilon,[\infty]}(t), Q_{\mathrm{d}}^{\varepsilon,[\infty]}(t)\right), \quad X_{\mathrm{d}}^{\varepsilon,[\ell]}(t)=\left(P_{\mathrm{d}}^{\varepsilon,[\ell]}(t), Q_{\mathrm{d}}^{\varepsilon,[\ell]}(t)\right),
$$

where $X_{\mathrm{d}}^{\varepsilon,[\infty]}(t)$ and $X_{\mathrm{d}}^{\varepsilon,[\ell]}(t)$ are as in point (i) and (ii) above, we recover the estimates

$$
\left|P_{\mathrm{d}}^{\varepsilon,[\infty]}(t)-P_{\mathrm{d}}^{\varepsilon}(t)\right|+\left|Q_{\mathrm{d}}^{\varepsilon,[\infty]}(t)-Q_{\mathrm{d}}^{\varepsilon}(t)\right| \leq C_{T} \exp \left(-\frac{\mu^{\prime}}{\varepsilon} t\right)
$$

and,

$$
\left|P_{\mathrm{d}}^{\varepsilon,[\ell]}(t)-P_{\mathrm{d}}^{\varepsilon}(t)\right|+\left|Q_{\mathrm{d}}^{\varepsilon,[\ell]}(t)-Q_{\mathrm{d}}^{\varepsilon}(t)\right| \leq C_{T} \varepsilon^{\ell+1}+C_{T} \exp \left(-\frac{\mu^{\prime}}{\varepsilon} t\right)
$$

whenever $t \in[0, T]$. 


\section{Proof of Theorem 4.3.}

It is a direct consequence of Theorem 3.2, in conjunction with the Perron-Frobenius Theorem 4.1.

\section{Analysis of the continuous model}

Recall that, with the notation (2.11) and (2.4)-(2.5), the continuous model (2.1) writes

$$
\left\{\begin{array}{l}
\frac{\partial p^{\varepsilon}}{\partial t}(t, x)=\frac{1}{\varepsilon} K_{p}\left(p^{\varepsilon}\right)(t, x)+F_{p}\left(p^{\varepsilon}(t, x), q^{\varepsilon}(t, x)\right), \\
\frac{\partial q^{\varepsilon}}{\partial t}(t, x)=\frac{1}{\varepsilon} K_{q}\left(q^{\varepsilon}\right)(t, x)+F_{q}\left(p^{\varepsilon}(t, x), q^{\varepsilon}(t, x)\right) \\
p^{\varepsilon}(0, x)=p_{0}(x), \quad q^{\varepsilon}(0, x)=q_{0}(x)
\end{array}\right.
$$

As in the previous section, the basic information we need to perform a central manifold analysis on the continuous model lies in identifying the kernel and spectral gaps of both operators $K_{p}$ and $K_{q}$.

\subsection{Equilibrium states of the transfer operator}

\subsubsection{Equilibrium states of the transfer operator in $L^{2}$}

Define for convenience

$$
\Sigma_{p}(x)=\int_{\Omega} \sigma_{p}(y, x) d y, \quad \Sigma_{q}(x)=\int_{\Omega} \sigma_{q}(y, x) d y .
$$

In this section, we study the kernel and spectral gap of the operator

$$
f \in L^{2}\left(\Omega ; \frac{\Sigma_{p}(x)}{p_{\mathrm{eq}}(x)} d x\right) \mapsto K_{p}(f) \in L^{2}\left(\Omega ; \frac{\Sigma_{p}(x)}{p_{\mathrm{eq}}(x)} d x\right),
$$

denoted by $K_{p}$ for simplicity in the sequel. The similar analysis is performed for the operator $K_{q}$, with the same short-hand notation. The role of the weight $\Sigma_{p}(x) / p_{\text {eq }}(x)$ (resp. $\left.\Sigma_{q}(x) / q_{\text {eq }}(x)\right)$ becomes clear below. Recall that the assumed positivity of $\sigma_{p}(x, y) \in$ $C^{0}(\Omega \times \Omega)$ and $\sigma_{q}(x, y) \in C^{0}(\Omega \times \Omega)$, together with the compactness of $\Omega$, ensure the existence of $\sigma_{\min }$ and $\sigma_{\max }$ such that

$$
\begin{array}{ll}
\forall(x, y) \in \Omega \times \Omega, \quad 0<\sigma_{\min } \leq \sigma_{p}(x, y) \leq \sigma_{\max }, \quad 0<\sigma_{\min } \leq \sigma_{q}(x, y) \leq \sigma_{\max }, \\
& 0<\sigma_{\min } \leq \Sigma_{p}(x) \leq \sigma_{\max }, \quad 0<\sigma_{\min } \leq \Sigma_{q}(x) \leq \sigma_{\max } .
\end{array}
$$

While in the finite dimensional case, the identification of the kernel and spectral gaps of $K_{p}$ and $K_{q}$ resulted from the application of the Perron-Frobenius theorem (under actually weaker assumptions of $\sigma_{p}$ and $\sigma_{q}$ than the present positivity requirement we need in the continuous case), the analogous analysis turns out to be much more difficult in the continuous situation.

We begin with the following Theorem. 


\section{Theorem 5.1. (Existence of an equilibrium state).}

(i) There exists a measurable function $p_{\mathrm{eq}}(x)$, which is almost everywhere positive, normalised in such a way that $\int_{\Omega} p_{\text {eq }}(x) d x=1$, which satisfies

$$
K_{p}\left(p_{\text {eq }}\right)(x)=0 \quad \text { a.e. } x \in \Omega .
$$

Besides, there exists a constant $M$ such that

$$
\int_{\Omega}\left(\frac{1}{\Sigma_{p}(x)}+\Sigma_{p}(x)\right) p_{\text {eq }}(x) d x \leq M .
$$

(ii) The function $p_{\mathrm{eq}}$ is continuous on $\Omega$. Hence there exist two constants $p_{\min }$ and $p_{\max }$ such that

$$
\forall x \in \Omega, \quad 0<p_{\min } \leq p_{\text {eq }}(x) \leq p_{\max } .
$$

(iii) Similarly, there exists a measurable function $q_{\mathrm{eq}}(x)$, which is almost everywhere positive, normalized in such a way that $\int_{\Omega} q_{\mathrm{eq}}(x) d x=1$, which satisfies

$$
K_{q}\left(q_{\mathrm{eq}}\right)(x)=0 \quad \text { a.e. } x \in \Omega \text {. }
$$

Besides, for a possibly larger value of the above constant $M$, we have

$$
\int_{\Omega}\left(\frac{1}{\Sigma_{q}(x)}+\Sigma_{q}(x)\right) q_{\mathrm{eq}}(x) d x \leq M
$$

The function $q_{\mathrm{eq}}$ is continuous on $\Omega$, and there exist two constants $q_{\min }$ and $q_{\max }$ such that

$$
\forall x \in \Omega, \quad 0<q_{\text {min }} \leq q_{\mathrm{eq}}(x) \leq q_{\max } .
$$

Remark. Naturally, the normalization $\int p_{\text {eq }}(x) d x=\int q_{\text {eq }}(x) d x=1$ ensures that $p_{\text {eq }}(x)$ resp. $q_{\mathrm{eq}}(x)$ describe the equilibrium repartition of preys resp. predators along the spatial variable $x$. The fact that $p_{\mathrm{eq}}$ and $q_{\mathrm{eq}}$ are positive is a consequence of the assumed positivity of the transfer rates $\sigma_{p}$ and $\sigma_{q}$ : the transfer operators do mix all populations, allowing for transfers from any site $x$ to any other site $y$.

\section{Proof of Theorem 5.1.}

The proof of point (i) is to be found in [DGP]. It is based on the Krein-Rutman theorem. It is actually proved in [DGP] that a sufficient condition for the existence of the equilibrium state $p_{\text {eq }}(x)$ is

$$
\sup _{x \in \Omega} \int_{\Omega} \frac{1+\Sigma_{p}(x)}{1+\Sigma_{p}(y)} \cdot \frac{\sigma_{p}(x, y)}{\Sigma_{p}(x)} d y<+\infty
$$

This bound is certainly true under our assumptions, since $\sigma_{p}$ is positive and continuous on a compact set. Hence the existence of $p_{\text {eq }}$ such that $K_{p}\left(p_{\text {eq }}\right)(x)=0$ a.e. $x$, or, in other words

$$
\Sigma_{p}(x) p_{\mathrm{eq}}(x)=\int_{\Omega} \sigma_{p}(x, y) p_{\mathrm{eq}}(y) d y, \quad \text { a.e. } x \in \Omega .
$$


To prove point (ii), we first write

$$
p_{\text {eq }}(x)=\frac{1}{\Sigma_{p}(x)} \int_{\Omega} \sigma_{p}(x, y) p_{\text {eq }}(y) d y
$$

a.e. $x$, hence, by continuity and positivity of $\sigma_{p}$, the function $p_{\text {eq }}(x)$ coincides with a continuous function. Naturally, the continuous function $p_{\text {eq }}$ cannot vanish, since in this case there would exist a point $x_{0}$ such that $p_{\text {eq }}\left(x_{0}\right)=0$, implying

$$
\int_{\Omega} \sigma_{p}\left(x_{0}, y\right) p_{\text {eq }}(y) d y=0
$$

and one would deduce $p_{\text {eq }}(y)=0$ for any $y$, the latter equlity being impossible due to the normalisation $\int_{\Omega} p_{\mathrm{eq}}=1$.

We are now in position to introduce, following [DGP], the key functional spaces of the present analysis, namely

$$
\begin{aligned}
& \mathcal{H}_{p}:=L^{2}\left(\Omega, \frac{\Sigma_{p}(x)}{p_{\mathrm{eq}}(x)} d x\right)=\left\{f: \Omega \rightarrow \mathbb{R}, \text { such that } f^{2} \frac{\Sigma_{p}}{p_{\mathrm{eq}}} \in L^{1}(\Omega)\right\}, \\
& \mathcal{H}_{q}:=L^{2}\left(\Omega, \frac{\Sigma_{q}(x)}{q_{\mathrm{eq}}(x)} d x\right)=\left\{f: \Omega \rightarrow \mathbb{R}, \text { such that } f^{2} \frac{\Sigma_{q}}{q_{\mathrm{eq}}} \in L^{1}(\Omega)\right\} .
\end{aligned}
$$

These are Hilbert spaces when endowed with the obvious scalar products

$$
\langle f, g\rangle_{\mathcal{H}_{p}}=\int_{\Omega} f(x) g(x) \frac{\Sigma_{p}(x)}{p_{\mathrm{eq}}(x)} d x, \quad\langle f, g\rangle_{\mathcal{H}_{q}}=\int_{\Omega} f(x) g(x) \frac{\Sigma_{q}(x)}{q_{\mathrm{eq}}(x)} d x .
$$

The equilibrium repartitions $p_{\text {eq }}$ and $q_{\text {eq }}$ naturally satisfy

$$
p_{\text {eq }}(x) \in \mathcal{H}_{p}, \quad q_{\text {eq }}(x) \in \mathcal{H}_{q},
$$

since $\left\|p_{\text {eq }}\right\|_{\mathcal{H}_{p}}=\left(\int_{\Omega} \Sigma_{p}(x) p_{\text {eq }}(x) d x\right)^{1 / 2}<\infty$, and similarly for the index $q$. Note also that the embeddings

$$
\mathcal{H}_{p} \subset L^{1}(\Omega), \quad \mathcal{H}_{q} \subset L^{1}(\Omega)
$$

are continuous, due to property (5.3). Indeed, we clearly have

$$
\int_{\Omega}|f(x)| d x \leq\|f\|_{\mathcal{H}_{p}}\left(\int_{\Omega} \frac{p_{\mathrm{eq}}(x)}{\Sigma_{p}(x)} d x\right)^{1 / 2},
$$

and similarly for the index $q$. One can thus define the closed subspace

$$
\mathcal{H}_{0}=\left\{f \in \mathcal{H}_{p} \text { such that } \int_{\Omega} f(x) d x=0\right\}=\left\{f \in \mathcal{H}_{q} \text { such that } \int_{\Omega} f(x) d x=0\right\} .
$$

Armed with these definition, we complete the picture given by Theorem 5.1, and provide the following spectral gap estimate 


\section{Theorem 5.2. (Spectral gap estimate)}

The following assertions hold true:

(i) Operator $K_{p}$ is continuous on $\mathcal{H}_{p}$.

(ii) The kernel of $K_{p}$ as an operator on $\mathcal{H}_{p}$ has dimension one. It is generated by the equilibrium function $p_{\text {eq }}$.

(iii) The following decomposition of $\mathcal{H}_{p}$ into closed subspaces, which are stable upon the action of $K_{p}$, holds true:

$$
\mathcal{H}_{p}=\operatorname{Vect} p_{\text {eq }} \oplus \mathcal{H}_{0} .
$$

(iv) There exist two positive constants $C_{p}>0$ and $\mu_{p}>0$ such that any solution $f(t, x) \in$ $C^{1}\left(\mathbb{R}^{+} ; \mathcal{H}_{0}\right)$ to the differential system $\frac{d}{d t} f(t, x)=K_{p}(f)(t, x)$ with initial datum $f(0, x) \in$ $\mathcal{H}_{0}$ satisfies

$$
\forall t \geq 0, \quad\|f(t)\|_{\mathcal{H}_{p}} \leq C_{p}\|f(0)\|_{\mathcal{H}_{p}} \exp \left(-\mu_{p} t\right) .
$$

(v) Similarly, operator $K_{q}$ is continuous over $\mathcal{H}_{q}$. The kernel of $K_{q}$ as an operator on $\mathcal{H}_{q}$ has dimension one. It is generated by the equilibrium function $q_{\mathrm{eq}}$. The following decomposition of $\mathcal{H}_{q}$ into closed subspaces, which are stable upon the action of $K_{q}$, holds true:

$$
\mathcal{H}_{q}=\operatorname{Vect} q_{\text {eq }} \oplus \mathcal{H}_{0} .
$$

Last, there exist two positive constants $C_{q}>0$ and $\mu_{q}>0$ such that any solution $f(t, x) \in$ $C^{1}\left(\mathbb{R}^{+} ; \mathcal{H}_{0}\right)$ to the differential system $\frac{d}{d t} f(t, x)=K_{q}(f)(t, x)$ with initial datum $f(0, x) \in$ $\mathcal{H}_{0}$ satisfies

$$
\forall t \geq 0, \quad\|f(t)\|_{\mathcal{H}_{q}} \leq C_{q}\|f(0)\|_{\mathcal{H}_{q}} \exp \left(-\mu_{q} t\right)
$$

The remainder part of this paragraph is devoted to the proof of Theorem 5.2. We essentially follow the approach developped in [DGP].

The proof is given through a series of Lemmas, which we state and prove below. The basic object whose study leads to the proof of Theorem 5.2 is the bilinear form

$$
B_{p}(f, g):=-\int_{\Omega} K_{p}(f)(x) g(x) \frac{d x}{p_{\text {eq }}(x)},
$$

defined over $\mathcal{H}_{p} \times \mathcal{H}_{p}$. The similar bilinear form is defined for the index "q". We readily stress the key fact that $B_{p}$ (and $B_{q}$ ) are not symmetric.

We begin with the

\section{Lemma 5.3. (Entropy estimate - see [DGP])}

The (non-symmetric) bilinear form $B_{p}$ is continuous over $\mathcal{H}_{p} \times \mathcal{H}_{p}$. The form is positive and satisfies the following entropy estimate, whenever $f \in \mathcal{H}_{p}$,

$$
\begin{aligned}
B_{p}(f, f) & =\frac{1}{2} \int_{\Omega \times \Omega}\left(\frac{f(x)}{p_{\mathrm{eq}}(x)}-\frac{f(y)}{p_{\mathrm{eq}}(y)}\right)^{2} \sigma_{p}(x, y) p_{\mathrm{eq}}(y) d x d y \\
& \geq \frac{1}{2}\left\|\left(\frac{1}{\Sigma_{p}}\right) K_{p}(f)\right\|_{\mathcal{H}_{p}}^{2} \geq 0 .
\end{aligned}
$$

The similar statement holds for $B_{q}$. 
Remark. The above Lemma is stated and proved in [DGP]. We reproduce its proof here for sake of completeness. The key idea behind this Lemma is the following: upon using the entropy inequality (5.9), one may prove point (iv) of Theorem 5.2, namely deduce the existence of a spectral gap associated with $K_{p}$, when viewed as an operator on $\mathcal{H}_{p}$. In a next step, we shall deduce from this spectral gap over $\mathcal{H}_{p}$ the existence of a (possibly smaller) spectral gap when $K_{p}$ is viewed as an operator on $C^{0}(\Omega)$. The latter will be the key ingredient in order to apply the central manifold Theorem to system (2.1).

\section{Proof of Lemma 5.3.}

Let us introduce the short-hand notation

$$
k_{p}(f)(x):=\int_{\Omega} \sigma_{p}(x, y) f(y) d y .
$$

The equilibrum relation $K_{p}\left(p_{\text {eq }}\right)(x)=0$ writes

$$
k_{p}\left(p_{\text {eq }}\right)(x)=\Sigma_{p}(x) p_{\text {eq }}(x) .
$$

Now, for any function $f \in \mathcal{H}_{p}$, we have

$$
\begin{aligned}
\|f\|_{\mathcal{H}_{p}}^{2} & =\int_{\Omega} \frac{f^{2}(x)}{p_{\mathrm{eq}}(x)} \Sigma_{p}(x) d x \\
& =\int_{\Omega} \int_{\Omega} \frac{f^{2}(x)}{p_{\mathrm{eq}}(x)} \sigma_{p}(y, x) d x d y=\int_{\Omega} \int_{\Omega} \frac{f^{2}(y)}{p_{\mathrm{eq}}(y)} \sigma_{p}(x, y) d x d y
\end{aligned}
$$

while relation (5.10) leads to

$$
\Sigma_{p}(x)=\int_{\Omega} \frac{p_{\text {eq }}(y)}{p_{\text {eq }}(x)} \sigma_{p}(x, y) d y .
$$

We recover in this way the identity

$$
\|f\|_{\mathcal{H}_{p}}^{2}=\int_{\Omega} \int_{\Omega} \frac{f^{2}(x)}{p_{\mathrm{eq}}(x)} \sigma_{p}(y, x) d x d y=\int_{\Omega} \int_{\Omega} \frac{f^{2}(x)}{p_{\mathrm{eq}}^{2}(x)} \sigma_{p}(x, y) p_{\mathrm{eq}}(y) d x d y .
$$

For positive functions $f$ and $g$, we may compute

$$
\begin{aligned}
& \int_{\Omega} k_{p}(f)(x) g(x) \frac{d x}{p_{\mathrm{eq}}(x)}=\int_{\Omega} \int_{\Omega} \sigma_{p}(x, y) f(y) g(x) \frac{d x d y}{p_{\mathrm{eq}}(x)} \\
& \leq\left(\int_{\Omega} \int_{\Omega} \sigma_{p}(x, y) f^{2}(y) \frac{d x d y}{p_{\mathrm{eq}}(y)}\right)^{1 / 2}\left(\int_{\Omega} \int_{\Omega} \sigma_{p}(x, y) g^{2}(y) \frac{p_{\mathrm{eq}}(y)}{p_{\mathrm{eq}}^{2}(x)} d x d y\right)^{1 / 2}
\end{aligned}
$$

from which, using (5.10) and (5.11), we deduce

$$
\int_{\Omega} k_{p}(f)(x) g(x) \frac{d x}{p_{\text {eq }}(x)} \leq\|f\|_{\mathcal{H}_{p}}\|g\|_{\mathcal{H}_{p}} .
$$

Since $B_{p}(f, g)=\langle f, g\rangle_{\mathcal{H}_{p}}-\int_{\Omega} k_{p}(f)(x) g(x) \frac{d x}{p_{\text {eq }}(x)}$, this establishes the coninuity of $B_{p}$ on $\mathcal{H}_{p} \times \mathcal{H}_{p}$ 
Incidentally, choosing $g=\frac{1}{\Sigma_{p}} k_{p}(f)$ in the above estimate, we readily deduce the continuity of the linear operator $f \in \mathcal{H}_{p} \mapsto \frac{1}{\Sigma_{p}} k_{p}(f) \in \mathcal{H}_{p}$, with a norm less than 1 .

For these reasons, using twice the equality $\frac{K_{p}(f)}{\Sigma_{p}(x)}=f(x)-\frac{k_{p}(f)}{\Sigma_{p}(x)}$, we deduce the estimate

$$
\begin{aligned}
B_{p}(f, f) & =\|f\|_{\mathcal{H}_{p}}^{2}-\left\langle\frac{1}{\Sigma_{p}} k_{p}(f), f\right\rangle_{\mathcal{H}_{p}}=\frac{1}{2}\left\|\frac{1}{\Sigma_{p}} K_{p}(f)\right\|_{\mathcal{H}_{p}}^{2}+\frac{1}{2}\left(\|f\|_{\mathcal{H}_{p}}^{2}-\left\|\frac{1}{\Sigma_{p}} k_{p}(f)\right\|_{\mathcal{H}_{p}}^{2}\right) \\
& \geq \frac{1}{2}\left\|\frac{1}{\Sigma_{p}} K_{p}(f)\right\|_{\mathcal{H}_{p}}^{2} \geq 0 .
\end{aligned}
$$

This establishes the lower bound in (5.9).

Last, to obtain the identity $B_{p}(f, f)=(1 / 2) \int_{\Omega \times \Omega} \ldots d x d y$ in (5.9), we write, using (5.11),

$$
\|f\|_{\mathcal{H}_{p}}^{2}=\frac{1}{2} \int_{\Omega} \int_{\Omega}\left(\frac{f^{2}(x)}{p_{\mathrm{eq}}^{2}(x)}+\frac{f^{2}(y)}{p_{\mathrm{eq}}^{2}(y)}\right) \sigma_{p}(x, y) p_{\mathrm{eq}}(y) d x d y
$$

On the other hand, we have

$$
\left\langle\frac{1}{\Sigma_{p}} k_{p}(f), f\right\rangle_{\mathcal{H}_{p}}=\int_{\Omega} \int_{\Omega} \frac{f(x)}{p_{\mathrm{eq}}(x)} \frac{f(y)}{p_{\mathrm{eq}}(y)} \sigma_{p}(x, y) p_{\mathrm{eq}}(y) d x d y
$$

Hence, we eventually arrive at

$$
B_{p}(f, f)=\|f\|_{\mathcal{H}_{p}}^{2}-\left\langle\frac{1}{\Sigma_{p}} k_{p}(f), f\right\rangle_{\mathcal{H}_{p}}=\frac{1}{2} \int_{\Omega} \int_{\Omega}\left(\frac{f(x)}{p_{\text {eq }}(x)}-\frac{f(y)}{p_{\text {eq }}(y)}\right)^{2} \sigma_{p}(x, y) p_{\text {eq }}(y) d x d y,
$$

which is the desired formula.

Lemma 5.3 is proved.

The following next Lemma will allow us, in conjunction with the above entropy estimate of Lemma 5.3, to complete the proof of Theorem 5.2.

\section{Lemma 5.4. (Coercivity estimate - see [DGP])}

There exists a constant $C_{p}>0$ such that

$$
\forall f \in \mathcal{H}_{p}, \quad B_{p}(f, f) \geq C_{p}\left\|f-\left(\int_{\Omega} f(y) d y\right) p_{\text {eq }}\right\|_{\mathcal{H}_{p}}^{2} .
$$

The analogous statement holds for the bilinear form $B_{q}$, namely, there exists $C_{q}>0$ such that

$$
\forall f \in \mathcal{H}_{q}, \quad B_{q}(f, f) \geq C_{q}\left\|f-\left(\int_{\Omega} f(y) d y\right) q_{\mathrm{eq}}\right\|_{\mathcal{H}_{q}}^{2}
$$




\section{Proof of Lemma 5.4.}

We again follow [DGP].

As the equilibrium function $p_{\text {eq }}(x)$ as well as the transfer rates $\Sigma_{p}(x)$ and $\sigma_{p}(x, y)$ are positive and continuous on the compact set $\Omega$ resp. $\Omega \times \Omega$, there exists a $\kappa>0$ such that

$$
\forall(x, y) \in \Omega \times \Omega, \quad p_{\mathrm{eq}}(x) \leq \kappa\left(\Sigma_{p}(y)+\frac{1}{\Sigma_{p}(y)}\right) \frac{1}{\Sigma_{p}(x)} \sigma_{p}(x, y) .
$$

For a given $f \in \mathcal{H}_{p}$, define now

$$
W_{p}(x, y):=\frac{f(y)}{p_{\text {eq }}(y)}-\frac{f(x)}{p_{\text {eq }}(x)} .
$$

Writing $f(x) p_{\text {eq }}(y)=f(y) p_{\text {eq }}(x)-p_{\text {eq }}(x) p_{\text {eq }}(y) W_{p}(x, y)$, and integrating with respect to $y$, gives

$$
f(x)-\left(\int_{\Omega} f(y) d y\right) p_{\mathrm{eq}}(x)=-p_{\mathrm{eq}}(x) \int_{\Omega} p_{\mathrm{eq}}(y) W_{p}(x, y) d y .
$$

The right-hand-side of the above equation may now be estimated upon using the CauchySchwarz inequality in conjunction with (5.3), providing

$$
\begin{aligned}
\mid p_{\mathrm{eq}}( & x)\left.\int_{\Omega} p_{\mathrm{eq}}(y) W_{p}(x, y) d y\right|^{2} \\
\leq & \left(\int_{\Omega}\left(\Sigma_{p}(y)+\frac{1}{\Sigma_{p}(y)}\right) p_{\mathrm{eq}}(y) d y\right)\left(\int_{\Omega} W_{p}^{2}(x, y) p_{\mathrm{eq}}^{2}(x) \frac{\Sigma_{p}(y)}{1+\Sigma_{p}(y)} p_{\mathrm{eq}}(y) d y\right) \\
\leq & M \int_{\Omega} W_{p}^{2}(x, y) p_{\mathrm{eq}}^{2}(x) \frac{\Sigma_{p}(y)}{1+\Sigma_{p}(y)} p_{\mathrm{eq}}(y) d y .
\end{aligned}
$$

We infer

$$
\left\|f-\left(\int_{\Omega} f(y) d y\right) p_{\mathrm{eq}}\right\|_{\mathcal{H}_{p}}^{2} \leq M \int_{\Omega} \int_{\Omega} W_{p}^{2}(x, y) p_{\mathrm{eq}}(x) \frac{\Sigma_{p}(y) \Sigma_{p}(x)}{1+\Sigma_{p}(y)} p_{\mathrm{eq}}(y) d y d x .
$$

Using the estimate (5.12) together with the identity (5.9), we obtain

$$
B_{p}(f, f)=\frac{1}{2} \int_{\Omega \times \Omega} W_{p}^{2}(x, y) \sigma_{p}(x, y) p_{\mathrm{eq}}(y) d x d y \geq \frac{1}{2 M \kappa}\left\|f-\left(\int_{\Omega} f(y) d y\right) p_{\mathrm{eq}}\right\|_{\mathcal{H}_{p}}^{2} .
$$

Lemma 5.4 is proved.

Having established Lemma 5.3 and Lemma 5.4, we conclude this paragraph upon proving Theorem 5.2.

\section{Proof of Theorem 5.2.}

Points (i), (ii) and (iii) are proved in the following way.

Using Lemma 5.3, we know the operators $f \in \mathcal{H}_{p} \mapsto \frac{1}{\Sigma_{p}} K_{p}(f) \in \mathcal{H}_{p}$ and $f \in \mathcal{H}_{p} \mapsto$ $K_{p}(f) \in \mathcal{H}_{p}$ are continuous. On top of that, in the case $K_{p}(f)=0$, we have $B_{p}(f, f)=0$ hence, as $\sigma_{p}$ is positive, the entropy dissipation estimate provides

$$
\frac{f(x)}{p_{\text {eq }}(x)}=\frac{f(y)}{p_{\text {eq }}(y)} \text { a.e. }(x, y) \text {. }
$$


In other words, the kernel of $K_{p}$ is seen to satisfy ker $K_{p}=\operatorname{Vect} p_{\text {eq }}$. Last, Vect $p_{\text {eq }}$ and $\mathcal{H}_{0}$ clearly have vanishing intersection, while Vect $p_{\text {eq }}$ obviously is stable upon the action of $K_{p}$, as is $\mathcal{H}_{0}$ due to the fact that operator $K_{p}$ is conservative.

Point (iv) of Theorem 5.2 now is obtained through the following argument.

Let $f_{0}(x) \in \mathcal{H}_{0}$ and $f \in \mathcal{C}^{1}\left(\mathbb{R}^{+} ; \mathcal{H}_{0}\right)$ be the solution to

$$
\frac{d}{d t} f(t, x)=K_{p}(f)(t, x), \quad f(0, x)=f_{0}(x) .
$$

We compute, using Lemma 5.4,

$$
\begin{gathered}
\frac{d}{d t}\left(\int_{\Omega}|f(t, x)|^{2} \frac{d x}{p_{\mathrm{eq}}(x)}\right)=\frac{d}{d t}\left\|\frac{f(t)}{\sqrt{\Sigma_{p}}}\right\|_{\mathcal{H}_{p}}^{2}=2\left\langle\frac{K_{p}(f)(t)}{\Sigma_{p}}, f(t)\right\rangle_{\mathcal{H}_{p}} \\
=-2 B_{p}(f(t), f(t)) \\
\leq-2 C_{p}\|f(t)\|_{\mathcal{H}_{p}}^{2} \leq-2 C_{p} \sigma_{\min }\left(\int_{\Omega}|f(t, x)|^{2} \frac{d x}{p_{\text {eq }}(x)}\right) .
\end{gathered}
$$

In other words, we have proved the key estimate

$$
\frac{d}{d t}\left(\int_{\Omega}|f(t, x)|^{2} \frac{d x}{p_{\text {eq }}(x)}\right) \leq-2 C_{p} \sigma_{\min }\left(\int_{\Omega}|f(t, x)|^{2} \frac{d x}{p_{\text {eq }}(x)}\right) .
$$

We deduce

$$
\forall t \geq 0, \quad \int_{\Omega}|f(t, x)|^{2} \frac{d x}{p_{\mathrm{eq}}(x)} \leq \exp \left(-2 C_{p} \sigma_{\min } t\right) \int_{\Omega}\left|f_{0}(x)\right|^{2} \frac{d x}{p_{\mathrm{eq}}(x)} .
$$

Hence

$$
\begin{aligned}
\|f(t)\|_{\mathcal{H}_{p}}^{2} & =\int_{\Omega}|f(t, x)|^{2} \frac{\Sigma_{p}(x)}{p_{\text {eq }}(x)} d x \\
& \leq \sigma_{\max } \int_{\Omega}|f(t, x)|^{2} \frac{d x}{p_{\text {eq }}(x)} \leq \sigma_{\max } \exp \left(-2 C_{p} \sigma_{\text {min }} t\right) \int_{\Omega}\left|f_{0}(x)\right|^{2} \frac{d x}{p_{\text {eq }}(x)} .
\end{aligned}
$$

This completes the proof of Theorem 5.2.

\section{Remark (location of $K_{p}$ 's spectrum).}

The key result of this paragraph is, the solution to $\frac{d}{d t} f(t, x)=K_{p}(f)(t, x)$, with initial datum $f(0, x)=f_{0} \in \mathcal{H}_{0}$ satisfies the exponential decay estimate of Theorem 5.2. Recall that in the finite dimensional setting, this piece of information had been obtained from the fact that $\sigma\left(\left.K_{p}\right|_{\mathcal{E}_{0}}\right) \subset\left\{z \in \mathbb{C}, \operatorname{Re}(z) \leq-\widetilde{\mu_{p}}<0\right\}$, for some $\widetilde{\mu_{p}}>0$. In the present case, since $K_{p}$ is not a symmetric operator, the above mentioned exponential decay estimate does not imply any information about the location of the spectrum of $K_{p}$, nor does the reverse implication hold. Yet the intermediate estimates that lead to Theorem 5.2 actually allow to locate the spectrum of $K_{p}$, as we now show. This fact illustrates the strength of the entropy estimate (5.3).

Let us show there exists a $\widetilde{\mu_{p}}>0$ such that $\sigma\left(\left.K_{p}\right|_{\mathcal{H}_{0}}\right) \subset\left\{z \in \mathbb{C}, \operatorname{Re}(z) \leq-\widetilde{\mu_{p}}<0\right\}$. To do so, consider $K_{p}$ as acting on $\mathcal{H}_{p, \mathbb{C}}$, the space obtained upon complexifying $\mathcal{H}_{p}$, and endowed with the complex scalar product

$$
\langle f, g\rangle_{\mathcal{H}_{p, \mathbb{C}}}:=\int_{\Omega} f(x) \overline{g(x)} \frac{\Sigma_{p}(x)}{p_{\text {eq }}(x)} d x .
$$


We define the closed subspace $\mathcal{H}_{0, \mathbb{C}}$ as $\mathcal{H}_{\mathbb{C}, 0}:=\left\{f \in \mathcal{H}_{p, \mathbb{C}}, \int_{\Omega} f(x) d x=0\right\}$. For any $\lambda \in \mathbb{C}$ and $h \in \mathcal{H}_{0, \mathbb{C}}$, we consider the problem

$$
\text { Find } f \in \mathcal{H}_{0, \mathbb{C}} \text { such that } K_{p}(f)-\lambda f=h \text {. }
$$

Formulating this question as a variational problem, we introduce the form $B_{p, \mathbb{C}}(f, g)=$ $-\int_{\Omega} K_{p}(f) \bar{g} \frac{d x}{p_{\text {eq }}}$, acting on $\mathcal{H}_{0, \mathbb{C}} \times \mathcal{H}_{0, \mathbb{C}}$. As in the proof of Theorem 5.2, we may establish that $B_{p, \mathbb{C}}$ is continuous on $\mathcal{H}_{0, \mathbb{C}} \times \mathcal{H}_{0, \mathbb{C}}$. Besides, there is a $C_{p}>0$ such that

$$
\forall f \in \mathcal{H}_{0, \mathbb{C}}, \quad \operatorname{Re}\left(B_{p, \mathbb{C}}(f, f)\right) \geq C_{p}\|f\|_{\mathcal{H}_{0, \mathbb{C}}}^{2}
$$

Define now the form $\mathcal{B}_{\lambda}(f, g):=B_{p, \mathbb{C}}(f, g)+\lambda\left\langle\frac{f}{\Sigma_{p}}, g\right\rangle_{\mathcal{H}_{0, \mathbb{C}}}$, defined on $\mathcal{H}_{0, \mathbb{C}} \times \mathcal{H}_{0, \mathbb{C}}$. From the above considerations, we know that $\mathcal{B}_{\lambda}$ is continuous on $\mathcal{H}_{0, \mathbb{C}} \times \mathcal{H}_{0, \mathbb{C}}$. Besides, there exists a $\widetilde{\mu_{p}}>0$ such that $\mathcal{B}_{\lambda}$ is coercitive whenever $-\widetilde{\mu_{p}}<\operatorname{Re}(\lambda)$. Therefore, the problem (5.14) also writes, in variational form,

$$
\text { Find } f \in \mathcal{H}_{0, \mathbb{C}}, \text { such that for any } g \in \mathcal{H}_{0, \mathbb{C}}, \quad \mathcal{B}_{\lambda}(f, g)=-\left\langle\frac{h}{\Sigma_{p}}, g\right\rangle_{\mathcal{H}_{0, \mathbb{C}}} .
$$

Now, Lax-Milgram's Theorem proves this equation admits a unique solution whenever $-\widetilde{\mu_{p}}<\operatorname{Re}(\lambda)$. As a consequence, $\sigma\left(\left.K_{p}\right|_{\mid \mathcal{H}_{0}}\right) \subset\left\{z \in \mathbb{C}, \operatorname{Re}(z) \leq-\widetilde{\mu_{p}}<0\right\}$, as claimed.

\subsubsection{Equilibrium states of the transfer operator in $C^{0}(\Omega)$}

This paragraph is devoted to transforming the spectral informations we derived in the previous paragraph on the operator $K_{p}$ when viewed as an operator on the appropriate weighted $L^{2}$ space, into spectral information on $K_{p}$ when viewed as an operator on $C^{0}(\Omega)$ (we use the same symbol $K_{p}$ for both operators not to overweight notation). This piece of information turns out to be the key ingredient when performing a central manifold analysis on the continuous model (2.1).

For later convenience, define the space

$$
\mathcal{E}_{0}:=\left\{f \in C^{0}(\Omega), \int_{\Omega} f(x) d x=0 .\right\} .
$$

This space plays the role of space $\mathcal{H}_{0}$ in the previous paragraph. Naturally, the obvious estimate $\left|\int_{\Omega} f(x) d x\right| \leq|\Omega|\|f\|_{L^{\infty}(\Omega)}$, where $|\Omega|$ denotes the Lebesgue mesure of $\Omega$, ensures $\mathcal{E}_{0}$ is a closed subspace of $C^{0}(\Omega)$.

We state the key result of the present section.

Theorem 5.5. The following assertions hold true:

(i) Operator $K_{p}$ is continuous on $C^{0}(\Omega)$.

(ii) The kernel of $K_{p}$ as an operator on $C^{0}(\Omega)$ has dimension one. It is generated by the equilibrium function $p_{\mathrm{eq}}$. 
(iii) The following decomposition of $C^{0}(\Omega)$ into closed subspaces, which are stable upon the action of $K_{p}$, holds true:

$$
C^{0}(\Omega)=\operatorname{Vect} p_{\text {eq }} \oplus \mathcal{E}_{0}
$$

(iv) There exist two positive constants ${ }^{4} C_{p}>0$ and $\mu_{p}>0$ such that any solution $f(t, x) \in$ $C^{1}\left(\mathbb{R}^{+} ; \mathcal{E}_{0}\right)$ to the differential system $\frac{d}{d t} f(t, x)=K_{p}(f)(t, x)$ with initial datum $f(0, x) \in \mathcal{E}_{0}$ satisfies

$$
\forall t \geq 0, \quad\|f(t)\|_{L^{\infty}(\Omega)} \leq C_{p}\|f(0)\|_{L^{\infty}(\Omega)} \exp \left(-\mu_{p} t\right)
$$

(v) Similarly, operator $K_{q}$ is continuous over $C^{0}(\Omega)$. The kernel of $K_{q}$ as an operator on $C^{0}(\Omega)$ has dimension one. It is generated by the equilibrium function $q_{\mathrm{eq}}$. The following decomposition of $C^{0}(\Omega)$ into closed subspaces, which are stable upon the action of $K_{q}$, holds true:

$$
C^{0}(\Omega)=\operatorname{Vect} q_{\mathrm{eq}} \oplus \mathcal{E}_{0}
$$

Last, there exist two positive constants $C_{q}>0$ and $\mu_{q}>0$ such that any solution $f(t, x) \in$ $C^{1}\left(\mathbb{R}^{+} ; C^{0}(\Omega)\right)$ to the differential system $\frac{d}{d t} f(t, x)=K_{q}(f)(t, x)$ with initial datum $f(0, x) \in$ $\mathcal{E}_{0}$ satisfies

$$
\forall t \geq 0, \quad\|f(t)\|_{L^{\infty}(\Omega)} \leq C_{q}\|f(0)\|_{L^{\infty}(\Omega)} \exp \left(-\mu_{q} t\right) .
$$

\section{Proof of Theorem 5.5.}

Point (i) is obvious.

To prove point (ii), take $f \in C^{0}(\Omega)$ such that $K_{p}(f)=0$. As

$$
C^{0}(\Omega) \subset \mathcal{H}_{p}=L^{2}\left(\Omega ; \frac{\Sigma_{p}(x)}{p_{\mathrm{eq}}(x)} d x\right),
$$

we readily deduce, using the results established in the previous paragraph, the equality $f(x)=\lambda p_{\text {eq }}(x)$ for some $\lambda \in \mathbb{R}$. The fact that $p_{\text {eq }} \in C^{0}$ allows to conclude.

Point (iii) is clear.

To prove point (iv), we exploit point (iv) from Theorem 5.2. Take $f(t, x)$ as in the statement of Theorem 5.5. As $C^{0}(\Omega) \subset \mathcal{H}_{p}=L^{2}\left(\Omega ; \frac{\Sigma_{p}(x)}{p_{\text {eq }}(x)} d x\right)$, we readily know from Theorem 5.2 that

$$
\begin{aligned}
\left(\int_{\Omega}|f(t, x)|^{2} \frac{\Sigma_{p}(x)}{p_{\mathrm{eq}}(x)} d x\right)^{1 / 2} & \leq C_{p} \exp \left(-\mu_{p} t\right)\left(\int_{\Omega}|f(0, x)|^{2} \frac{\Sigma_{p}(x)}{p_{\mathrm{eq}}(x)} d x\right)^{1 / 2} \\
& \leq C_{p}|\Omega|^{1 / 2} \frac{\sigma_{\mathrm{max}}^{1 / 2}}{p_{\min }^{1 / 2}} \exp \left(-\mu_{p} t\right)\|f(0)\|_{L^{\infty}(\Omega)} .
\end{aligned}
$$

Eventually, we may write

$$
\left(\int_{\Omega}|f(t, x)|^{2} d x\right)^{1 / 2} \leq C_{p}^{\prime} \exp \left(-\mu_{p} t\right)\|f(0)\|_{L^{\infty}(\Omega)},
$$

\footnotetext{
${ }^{4}$ We use the same constants $C_{p}$ and $\mu_{p}$ as in the statement of Theorem 5.2 not to overweight notation
} 
upon setting $C_{p}^{\prime}=C_{p}|\Omega|^{1 / 2}\left(\sigma_{\max } / \sigma_{\min }\right)^{1 / 2}\left(p_{\max } / p_{\min }\right)^{1 / 2}$. Once more using the equation that defines $f(t, x)$, we obtain

$$
\begin{aligned}
\frac{\partial f}{\partial t}(t, x) & =\int_{\Omega} \sigma_{p}(x, y) f(t, y) d y-\Sigma_{p}(x) f(t, x) \\
& \leq \sigma_{\max }|\Omega|^{1 / 2} C_{p}^{\prime} \exp \left(-\mu_{p} t\right)\|f(0)\|_{L^{\infty}(\Omega)}-\Sigma_{p}(x) f(t, x) .
\end{aligned}
$$

Therefore, we recover, upon possibly taking a smaller value of $\mu_{p}$,

$$
\begin{aligned}
f(t, x) \leq & f(0, x) \exp \left(-\Sigma_{p}(x) t\right) \\
& +\frac{\sigma_{\max }|\Omega|^{1 / 2} C_{p}^{\prime}\|f(0)\|_{L^{\infty}(\Omega)}}{\Sigma_{p}(x)-\mu_{p}}\left[\exp \left(-\mu_{p} t\right)-\exp \left(-\Sigma_{p}(x) t\right)\right] .
\end{aligned}
$$

Using the information $\Sigma_{p} \geq \sigma_{\min }>0$ completes the proof of statement (iv).

Remark. Using the previously established fact that $\sigma\left(\left.K_{p}\right|_{\mathcal{H}_{0}}\right) \subset\left\{z \in \mathbb{C}, \operatorname{Re}(z) \leq-\widetilde{\mu_{p}}<\right.$ $0\}$ for some $\widetilde{\mu_{p}}>0$, one may similarly prove that $\sigma\left(K_{p} \mid \mathcal{E}_{0}\right) \subset\left\{z \in \mathbb{C}, \operatorname{Re}(z) \leq-\widetilde{\mu_{p}}<0\right\}$ for some possibly smalller $\widetilde{\mu_{p}}>0$.

\subsection{Writing the continuous model in slow-fast form}

As in the discrete case, Theorem 5.5 allows to identify the "good" slow and fast variables in the original model (2.1). Indeed, associated with the decompositions

$$
C^{0}(\Omega)=\operatorname{Vect} p_{\text {eq }} \oplus \mathcal{E}_{0}, \quad \text { and } C^{0}(\Omega)=\operatorname{Vect} q_{\text {eq }} \oplus \mathcal{E}_{0},
$$

one may split the solution to (2.1) into

$$
\begin{aligned}
& p^{\varepsilon}(t, x)=P_{\mathrm{c}}^{\varepsilon}(t) p_{\mathrm{eq}}(x)+R_{p}^{\varepsilon}(t, x) \in \operatorname{Vect} p_{\mathrm{eq}} \oplus \mathcal{E}_{0}, \\
& q^{\varepsilon}(t, x)=Q_{\mathrm{c}}^{\varepsilon}(t) q_{\mathrm{eq}}(x)+R_{q}^{\varepsilon}(t, x) \in \operatorname{Vect} q_{\mathrm{eq}} \oplus \mathcal{E}_{0},
\end{aligned}
$$

where $P_{\mathrm{c}}^{\varepsilon}(t), Q_{\mathrm{c}}^{\varepsilon}(t), R_{p}^{\varepsilon}(t, x)$ and $R_{q}^{\varepsilon}(t, x)$ have the obvious values

$$
\begin{aligned}
& P_{\mathrm{c}}^{\varepsilon}(t)=\int_{\Omega} p^{\varepsilon}(t, x) d x=: S p^{\varepsilon}(t), \quad R_{p}^{\varepsilon}(t, x)=p^{\varepsilon}(t, x)-P_{\mathrm{c}}^{\varepsilon}(t) p_{\mathrm{eq}}(x)=: R_{p} p^{\varepsilon}(t, x), \\
& \left.Q_{\mathrm{c}}^{\varepsilon}(t)=\int_{\Omega} q^{\varepsilon}(t, x) d x=: S q^{\varepsilon}(t), \quad R_{q}^{\varepsilon}(t, x)=q^{\varepsilon}(t, x)-Q_{\mathrm{c}}^{\varepsilon}(t) q_{\mathrm{eq}}(x)\right)=: R_{q} q^{\varepsilon}(t, x),
\end{aligned}
$$

and the above equation implicitely defines the notation, valid for any $p(x) \in C^{0}(\Omega)$,

$$
S p:=\int_{\Omega} p(x) d x, R_{p} p(x):=p(x)-(S p) p_{\mathrm{eq}}(x), R_{q} p(x):=q(x)-(S q) q_{\mathrm{eq}}(x) .
$$

Naturally, the two changes of unknowns $p^{\varepsilon}(t, x) \in C^{0}(\Omega) \mapsto\left(P_{\mathrm{c}}^{\varepsilon}(t), R_{p}^{\varepsilon}(t, x)\right) \in \mathbb{R} \times \mathcal{E}_{0}$ and $q^{\varepsilon}(t, x) \in C^{0}(\Omega) \mapsto\left(Q_{\mathrm{c}}^{\varepsilon}(t), R_{q}^{\varepsilon}(t, x)\right) \in \mathbb{R} \times \mathcal{E}_{0}$ are one-to-one. Again, the explicit value of the equilibrium vectors $p_{\mathrm{eq}}$ and $q_{\mathrm{eq}}$ cannot be given in any general form: it has to be computed for each specific model independently, and similarly for the above changes of unknowns. 
With these new variables, the continuous system (2.1) writes

$$
\left\{\begin{array}{l}
\frac{d}{d t} P_{\mathrm{c}}^{\varepsilon}(t)=S F_{p}\left(P_{\mathrm{c}}^{\varepsilon}(t) p_{\mathrm{eq}}(x)+R_{p}^{\varepsilon}(t, x), Q_{\mathrm{c}}^{\varepsilon}(t) q_{\mathrm{eq}}(x)+R_{q}^{\varepsilon}(t, x)\right) \\
\frac{d}{d t} Q_{\mathrm{c}}^{\varepsilon}(t)=S F_{q}\left(P_{\mathrm{c}}^{\varepsilon}(t) p_{\mathrm{eq}}(x)+R_{p}^{\varepsilon}(t, x), Q_{\mathrm{c}}^{\varepsilon}(t) q_{\mathrm{eq}}(x)+R_{q}^{\varepsilon}(t, x)\right) \\
\frac{d}{d t} \mathbf{R}_{p}^{\varepsilon}(t, x)=\frac{1}{\varepsilon} K_{p}\left(R_{p}^{\varepsilon}\right)(t, x)+R_{p} F_{p}\left(P_{\mathrm{c}}^{\varepsilon}(t) p_{\mathrm{eq}}(x)+R_{p}^{\varepsilon}(t, x), Q_{\mathrm{c}}^{\varepsilon}(t) q_{\mathrm{eq}}(x)+R_{q}^{\varepsilon}(t, x)\right) \\
\frac{d}{d t} \mathbf{R}_{q}^{\varepsilon}(t, x)=\frac{1}{\varepsilon} K_{q}\left(R_{q}^{\varepsilon}\right)(t, x)+R_{q} F_{q}\left(P_{\mathrm{c}}^{\varepsilon}(t) p_{\mathrm{eq}}(x)+R_{p}^{\varepsilon}(t, x), Q_{\mathrm{c}}^{\varepsilon}(t) q_{\mathrm{eq}}(x)+R_{q}^{\varepsilon}(t, x)\right), \\
P_{\mathrm{c}}^{\varepsilon}(0, x)=S p_{0}(x), \quad Q_{\mathrm{c}}^{\varepsilon}(0, x)=S q_{0}(x), \quad \mathbf{R}_{p}^{\varepsilon}(0, x)=R_{p} p_{0}(x), \quad \mathbf{R}_{q}^{\varepsilon}(0, x)=R_{q} q_{0}(x) .
\end{array}\right.
$$

where we use the notation (5.17) and the nonlinear functions $F_{p}$ and $F_{q}$ (the Lotka-Volterra part of the population dynamics under study) have been defined in (2.11).

System (5.19) now is in the necessary slow-fast form required to apply the central manifold theorem. Indeed, setting ${ }^{5}$

$$
X_{\mathrm{c}}^{\varepsilon}(t)=\left(P_{\mathrm{c}}^{\varepsilon}(t), Q_{\mathrm{c}}^{\varepsilon}(t)\right) \in \mathbb{R}^{2}, \text { and } Y_{\mathrm{c}}^{\varepsilon}(t)=\left(R_{p}^{\varepsilon}(t, x), R_{q}^{\varepsilon}(t, x) \in \mathcal{E}_{0} \times \mathcal{E}_{0} .\right.
$$

together with

$$
\begin{aligned}
& f_{0, \mathrm{c}}\left(X_{\mathrm{c}}^{\varepsilon}, Y_{\mathrm{c}}^{\varepsilon}\right)=\left(\begin{array}{c}
S F_{p}\left(P_{\mathrm{c}}^{\varepsilon}(t) p_{\mathrm{eq}}(x)+R_{p}^{\varepsilon}(t, x), Q_{\mathrm{c}}^{\varepsilon}(t) q_{\mathrm{eq}}(x)+R_{q}^{\varepsilon}(t, x)\right) \\
S F_{q}\left(P_{\mathrm{c}}^{\varepsilon}(t) p_{\mathrm{eq}}(x)+R_{p}^{\varepsilon}(t, x), Q_{\mathrm{c}}^{\varepsilon}(t) q_{\mathrm{eq}}(x)+R_{q}^{\varepsilon}(t, x)\right)
\end{array}\right), \\
& K Y_{\mathrm{c}}^{\varepsilon}=\left(\begin{array}{c}
K_{p}\left(R_{p}^{\varepsilon}\right)(t, x) \\
K_{q}\left(R_{q}^{\varepsilon}\right)(t, x)
\end{array}\right), \\
& g_{1, \mathrm{c}}\left(X_{\mathrm{c}}^{\varepsilon}, Y_{\mathrm{c}}^{\varepsilon}\right)=\left(\begin{array}{c}
R_{p} F_{p}\left(P_{\mathrm{c}}^{\varepsilon}(t) p_{\mathrm{eq}}(x)+R_{p}^{\varepsilon}(t, x), Q_{\mathrm{c}}^{\varepsilon}(t) q_{\mathrm{eq}}(x)+R_{q}^{\varepsilon}(t, x)\right) \\
R_{q} F_{q}\left(P_{\mathrm{c}}^{\varepsilon}(t) p_{\mathrm{eq}}(x)+R_{p}^{\varepsilon}(t, x), Q_{\mathrm{c}}^{\varepsilon}(t) q_{\mathrm{eq}}(x)+R_{q}^{\varepsilon}(t, x)\right)
\end{array}\right),
\end{aligned}
$$

system (5.19) takes the form

$$
\left(S_{\varepsilon, \mathrm{c}}\right)\left\{\begin{array}{l}
\frac{d}{d t} X_{\mathrm{c}}^{\varepsilon}(t)=f_{0, \mathrm{c}}\left(X_{\mathrm{c}}^{\varepsilon}(t), Y_{\mathrm{c}}^{\varepsilon}(t), \varepsilon\right), \quad X_{\mathrm{c}}^{\varepsilon}(0)=x_{0}, \\
\frac{d}{d t} Y_{\mathrm{c}}^{\varepsilon}(t)=\frac{K}{\varepsilon} Y_{\mathrm{c}}^{\varepsilon}(t)+g_{1, \mathrm{c}}\left(X_{\mathrm{c}}^{\varepsilon}(t), Y_{\mathrm{c}}^{\varepsilon}(t), \varepsilon\right), \quad Y_{\mathrm{c}}^{\varepsilon}(0)=y_{0} .
\end{array}\right.
$$

We are in position to apply the central manifold Theorem 3.1 to system $\left(S_{\varepsilon, \mathrm{c}}\right)$.

Before doing so, and for sake of completeness, we readily state the following necessary Lemma.

Lemma 5.6. The nonlinear functions $f_{0, \mathrm{c}}$ and $g_{1, \mathrm{c}}$ defined in (5.21) and (5.23) have $C^{\infty}$ smoothness when acting on $\mathbb{R}^{2} \times \mathcal{E}_{0}^{2}$.

\section{Proof of Lemma 5.6.}

The only difficulty lies in the presence of linear operators $S, R_{p}$, and $R_{q}$ in the definition of both nonlinear functions.

\footnotetext{
${ }^{5}$ The lower index "c" again refers to the continuous system
} 
Let us check for instance that $f_{0, c}$ is continuous on $\mathbb{R}^{2} \times \mathcal{E}_{0}^{2}$. Checking so actually is enough since $f_{0, \mathrm{c}}$ is a polynomial, hence checking that higher (Fréchet) derivatives of $f_{0, \mathrm{c}}$ are continuous follows the same lines. To prove continuity of $f_{0, \mathrm{c}}$, we establish this function is locally Lipschitz. Hence, taking $(X, Y) \in \mathbb{R}^{2} \times \mathcal{E}_{0}^{2}$ and $\left(X^{\prime}, Y^{\prime}\right) \in \mathbb{R} \times \mathcal{E}_{0}$ both belonging to some compact subset $\mathcal{K}$ of $\mathbb{R}^{2} \times \mathcal{E}_{0}^{2}$, and using the notation

$$
\begin{aligned}
& X=(P, Q) \in \mathbb{R}^{2}, \quad Y=\left(R_{p}(x), R_{q}(x)\right) \in \mathcal{E}_{0}^{2}, \\
& X^{\prime}=\left(P^{\prime}, Q^{\prime}\right) \in \mathbb{R}^{2}, \quad Y^{\prime}=\left(R_{p}^{\prime}(x), R_{q}^{\prime}(x)\right) \in \mathcal{E}_{0}^{2},
\end{aligned}
$$

we write,

$$
\begin{aligned}
\left|f_{0, \mathrm{c}}(X, Y)-f_{0, \mathrm{c}}\left(X^{\prime}, Y^{\prime}\right)\right| \leq \\
\mid S\left(F_{p}\left(P p_{\mathrm{eq}}(x)+R_{p}(x), Q q_{\mathrm{eq}}(x)+R_{q}(x)\right)\right. \\
\left.\quad-F_{p}\left(P^{\prime} p_{\mathrm{eq}}(x)+R_{p}^{\prime}(x), Q^{\prime} q_{\mathrm{eq}}(x)+R_{q}^{\prime}(x)\right)\right) \mid \\
+\mid S\left(F_{q}\left(P p_{\mathrm{eq}}(x)+R_{p}(x), Q q_{\mathrm{eq}}(x)+R_{q}(x)\right)\right. \\
\left.\quad-F_{q}\left(P^{\prime} p_{\mathrm{eq}}(x)+R_{p}^{\prime}(x), Q^{\prime} q_{\mathrm{eq}}(x)+R_{q}^{\prime}(x)\right)\right) \mid .
\end{aligned}
$$

Hence, using the obvious estimate

$$
\forall Z \in C^{0}(\Omega), \quad|S Z| \leq\|Z\|_{L^{1}(\Omega)} \leq|\Omega|\|Z\|_{L^{\infty}(\Omega)},
$$

(see the definition (5.18) of $S$ ), together with the fact that $F_{p}$ and $F_{q}$ are locally Lipschitz on $C^{0}(\Omega) \times C^{0}(\Omega)$, with a Lipschitz constant $C(\mathcal{K})$ that depends on the compact set $\mathcal{K}$, we recover

$$
\begin{aligned}
& \left|f_{0, \mathrm{c}}(X, Y)-f_{0, \mathrm{c}}\left(X^{\prime}, Y^{\prime}\right)\right| \leq|\Omega| \times C(\mathcal{K}) \times \\
& \quad\left(\left\|\left[P-P^{\prime}\right] p_{\mathrm{eq}}(x)+\left[R_{p}(x)-R_{p}^{\prime}(x)\right]\right\|_{L^{\infty}(\Omega)}+\left\|\left[Q-Q^{\prime}\right] q_{\mathrm{eq}}(x)+\left[R_{q}(x)-R_{q}^{\prime}(x)\right]\right\|_{L^{\infty}(\Omega)}\right) .
\end{aligned}
$$

Hence, using the continuity of both $p_{\mathrm{eq}}(x)$ and $q_{\mathrm{eq}}(x)$, we eventually obtain

$$
\begin{aligned}
& \left|f_{0, \mathrm{c}}(X, Y)-f_{0, \mathrm{c}}\left(X^{\prime}, Y^{\prime}\right)\right| \leq|\Omega| \times C(\mathcal{K}) \times \\
& \quad\left(\left|P-P^{\prime}\right|\left\|p_{\mathrm{eq}}\right\|_{L^{\infty}(\Omega)}+\left|Q-Q^{\prime}\right|\left\|q_{\mathrm{eq}}\right\|_{L^{\infty}(\Omega)}+\left\|R_{p}-R_{p}^{\prime}\right\|_{L^{\infty}(\Omega)}+\left\|R_{q}-R_{q}^{\prime}\right\|_{L^{\infty}(\Omega)}\right),
\end{aligned}
$$

and $f_{0, \mathrm{c}}$ is seen to be locally Lipschitz. Repeating the argument for $g_{1, \mathrm{c}}$, next for the higher order derivatives of $f_{0, c}$ and $g_{1, \mathrm{c}}$, proves the Lemma.

\subsection{Application of the central manifold Theorem}

When written in the form $\left(S_{\varepsilon, \mathrm{c}}\right)$, the continuous system (2.1) clearly satisfies the assumptions of the central manifold Theorem 3.1, as well as those of Theorem 3.2, with $E=\mathbb{R} \times \mathbb{R}$, $F=\mathcal{E}_{0} \times \mathcal{E}_{0}$, and $r$ may be any integer (the functions at hand actually have $C^{\infty}$ smoothness). Again, strictly speaking, the relevant functions $f_{0, \mathrm{c}}$ and $g_{1, \mathrm{c}}$ involved in $\left(S_{\varepsilon, \mathrm{c}}\right)$ are not bounded over the whole of $E \times F$, yet this is a harmless point. Recall that both linear operators $K_{p}$ and $K_{q}$ are bijections from $\mathcal{E}_{0}$ to itself, hence the linear operator $K$ in (4.14) is a bijection from $\mathcal{E}_{0} \times \mathcal{E}_{0}$ to itself. Recall also that the various entropy estimates of paragraph 5.1.1 (which somehow replace the lack of a Perron-Frobenius theorem in the infinite 
dimensional situation), and more precisely estimate (5.13), show that the linear operator $K$ satisfies

$$
\forall Y \in \mathcal{E}_{0} \times \mathcal{E}_{0}, \quad\left\|\exp \left(K \frac{t}{\varepsilon}\right) Y\right\|_{\mathcal{E}_{0}^{2}} \leq \exp \left(-\mu \frac{t}{\varepsilon}\right)\|Y\|_{\mathcal{E}_{0}^{2}},
$$

for some $C>0$ and $\mu>0$.

Now, the central manifold theorem asserts the existence of

$$
h_{\mathrm{c}}(X, \varepsilon)=\varepsilon h_{1, \mathrm{c}}(X)+\varepsilon^{2} h_{2, \mathrm{c}}(X)+\cdots
$$

such that Theorem 3.1 holds true when applied to the continuous system (5.19) or equivalently $\left(S_{\varepsilon, \mathrm{c}}\right)$. Associated with the function $h_{\mathrm{c}}(X, \varepsilon)$, one may write the reduced dynamics

$$
\left(S_{\varepsilon, \mathrm{c}}^{[\infty]}\right) \quad \frac{d}{d t} X_{\mathrm{c}}^{\varepsilon,[\infty]}(t)=f_{0, \mathrm{c}}\left(X_{\mathrm{c}}^{\varepsilon,[\infty]}(t), h\left(X_{\mathrm{c}}^{\varepsilon,[\infty]}(t), \varepsilon\right), \varepsilon\right), \quad Y_{\mathrm{c}}^{\varepsilon,[\infty]}(0)=h_{\mathrm{c}}\left(X_{\mathrm{c}}^{\varepsilon,[\infty]}(t), \varepsilon\right),
$$

as well as, for any integer $\ell$, the truncated versions of $\left(S_{\varepsilon, \mathrm{c}}^{[\infty]}\right)$, namely

$$
\left(S_{\varepsilon, \mathrm{c}}^{[\ell]}\right) \quad \frac{d}{d t} X_{\mathrm{c}}^{\varepsilon,[\ell]}(t)=f_{0, \mathrm{c}}\left(X_{\mathrm{c}}^{\varepsilon,[\ell]}(t), h^{[\ell]}\left(X_{\mathrm{c}}^{\varepsilon,[\ell]}(t), \varepsilon\right), \varepsilon\right), \quad Y_{\mathrm{c}}^{\varepsilon,[\ell]}(0)=h_{\mathrm{c}}\left(X_{\mathrm{c}}^{\varepsilon,[\ell]}(t), \varepsilon\right),
$$

as we did in the previous paragraph.

To complete our analysis we now compute the functions $h_{1, \mathrm{c}}, h_{2, \mathrm{c}}$, etc., and study the properties of the reduced models $\left(S_{\varepsilon, \mathrm{c}}^{[\infty]}\right)$ and $\left(S_{\varepsilon, \mathrm{c}}^{[\ell]}\right)$.

\subsubsection{Computing the reduced models}

To compute the actual values of $h_{1, \mathrm{c}}, h_{2, \mathrm{c}}$, etc. we argue as in paragraph 4.3 .1 , and write down the fact that vectors of the form $Y=h_{\mathrm{c}}(X, \varepsilon)$ are left invariant by the flow of $\left(S_{\varepsilon, \mathrm{c}}\right)$. We skip the details, and simply give the final formulae

$$
\begin{aligned}
h_{1, \mathrm{c}}(X)= & -K^{-1} g_{1, \mathrm{c}}(X, 0), \\
h_{2, \mathrm{c}}(X)= & K^{-1}\left[D_{X} h_{1, \mathrm{c}}(X) f_{0, \mathrm{c}}(X, 0)-D_{Y} g_{1, \mathrm{c}}(X, 0) h_{1, \mathrm{c}}(X)\right], \\
h_{3, \mathrm{c}}(X)= & K^{-1}\left[D_{X} h_{2, \mathrm{c}}(X) f_{0, \mathrm{c}}(X, 0)+D_{X} h_{1, \mathrm{c}}(X) D_{Y} f_{0, \mathrm{c}}(X, 0) h_{1, \mathrm{c}}(X)\right. \\
& +\frac{1}{2} D_{Y, Y}^{2} f_{0, \mathrm{c}}(X, 0) h_{1, \mathrm{c}}(X) h_{1, \mathrm{c}}(X)-D_{Y} g_{1, \mathrm{c}}(X, 0) h_{2, \mathrm{c}}(X) \\
& \left.-\frac{1}{2} D_{Y, Y}^{2} g_{1, \mathrm{c}}(X, 0) h_{1, \mathrm{c}}(X) h_{1, \mathrm{c}}(X)\right]
\end{aligned}
$$

and so on, where $K^{-1}$ is the inverse of $K$ as an operator on $\mathcal{E}_{0} \times \mathcal{E}_{0}$, and $D_{X}, D_{Y}$, denote the Fréchet derivatives with respect to $X$ resp. $Y$. The reduced models $\left(S_{\varepsilon, \mathrm{c}}^{[\ell]}\right)$ naturally obtained upon applying the central manifold theorem to system (2.1) may now be explicitely written down, for any value of $\ell$. For definiteness we only give here the explicit values for the values $\ell=0,1$, as based on the previous explicit computations.

Theorem 5.7. (i) the zeroth order reduced dynamics $\left(S_{\varepsilon, \mathrm{c}}^{[0]}\right)$ provided by the central manifold Theorem has the explicit value

$$
\left(S_{\varepsilon, \mathrm{c}}^{[0]}\right) \quad\left\{\begin{aligned}
\frac{d}{d t} P_{\mathrm{c}}^{\varepsilon,[0]}(t) & =A_{p}^{0} P_{\mathrm{c}}^{\varepsilon,[0]}(t)-B_{p}^{0} P_{\mathrm{c}}^{\varepsilon,[0]}(t) Q_{\mathrm{c}}^{\varepsilon,[0]}(t), \\
\frac{d}{d t} Q_{\mathrm{c}}^{\varepsilon,[0]}(t) & =-A_{q}^{0} Q_{\mathrm{c}}^{\varepsilon,[0]}(t)+B_{q}^{0} P_{\mathrm{c}}^{\varepsilon,[0]}(t) Q_{\mathrm{c}}^{\varepsilon,[0]}(t),
\end{aligned}\right.
$$


where the positive coefficients $A_{p}^{0}, B_{p}^{0}, A_{q}^{0}, B_{q}^{0}$, have the values

$$
\begin{array}{ll}
A_{p}^{0}=S\left(a_{p}(x) p_{\mathrm{eq}}(x)\right), & B_{p}^{0}=S\left(b_{p}(x) p_{\mathrm{eq}}(x) q_{\mathrm{eq}}(x)\right), \\
A_{q}^{0}=S\left(a_{q}(x) q_{\mathrm{eq}}(x)\right), & B_{q}^{0}=S\left(b_{q}(x) p_{\mathrm{eq}}(x) q_{\mathrm{eq}}(x)\right) .
\end{array}
$$

(ii) the first order reduced dynamics $\left(S_{\varepsilon, \mathrm{c}}^{[1]}\right)$ provided by the central manifold Theorem has the explicit value

$$
\left(S_{\varepsilon, \mathrm{c}}^{[1]}\right)\left\{\begin{aligned}
\frac{d}{d t} P_{\mathrm{c}}^{\varepsilon,[1]}(t)= & \left(A_{p}^{0}+\varepsilon A_{p}^{1}\right) P_{\mathrm{c}}^{\varepsilon,[1]}(t)-\left(B_{p}^{0}+\varepsilon B_{p}^{1}\right) P_{\mathrm{c}}^{\varepsilon,[1]}(t) Q_{\mathrm{c}}^{\varepsilon,[1]}(t) \\
& +\varepsilon C_{p}^{1}\left(P_{\mathrm{c}}^{\varepsilon,[1]}(t)\right)^{2} Q_{\mathrm{c}}^{\varepsilon,[1]}(t)+\varepsilon D_{p}^{1} P_{\mathrm{c}}^{\varepsilon,[1]}(t)\left(Q_{\mathrm{c}}^{\varepsilon,[1]}(t)\right)^{2} \\
\frac{d}{d t} Q_{\mathrm{c}}^{\varepsilon,[1]}(t)=- & \left(A_{q}^{0}+\varepsilon A_{q}^{1}\right) Q_{\mathrm{c}}^{\varepsilon,[1]}(t)+\left(B_{q}^{0}+\varepsilon B_{q}^{1}\right) P_{\mathrm{c}}^{\varepsilon,[1]}(t) Q_{\mathrm{c}}^{\varepsilon,[0]}(t) \\
& +\varepsilon C_{q}^{1}\left(P_{\mathrm{c}}^{\varepsilon,[1]}(t)\right)^{2} Q_{\mathrm{c}}^{\varepsilon,[1]}(t)+\varepsilon D_{q}^{1} P_{\mathrm{c}}^{\varepsilon,[1]}(t)\left(Q_{\mathrm{c}}^{\varepsilon,[1]}(t)\right)^{2}
\end{aligned}\right.
$$

where the positive coefficients $A_{p}^{0}, B_{p}^{0}, A_{q}^{0}, B_{q}^{0}$ are defined before while

$$
\begin{aligned}
& A_{p}^{1}=S\left(a_{p}(x) \widetilde{a_{p}}(x)\right), \quad A_{q}^{1}=S\left(a_{q}(x) \widetilde{a_{q}(x)}\right), \\
& B_{p}^{1}=S\left(-a_{p}(x) \widetilde{b_{p}(x)}+b_{p}(x) \widetilde{a_{q}(x)} p_{\mathrm{eq}}(x)+b_{p}(x) \widetilde{a_{p}(x)} q_{\mathrm{eq}}(x)\right), \\
& B_{q}^{1}=S\left(-a_{q}(x) \widetilde{b_{q}(x)}+b_{q}(x) \widetilde{a_{p}(x)} q_{\mathrm{eq}}(x)+b_{q}(x) \widetilde{a_{q}(x)} p_{\mathrm{eq}}(x)\right), \\
& C_{p}^{1}=-S\left(b_{p}(x) \widetilde{b_{q}(x)} p_{\mathrm{eq}}(x)\right), \quad D_{p}^{1}=-S\left(b_{p}(x) \widetilde{b_{p}(x)} q_{\mathrm{eq}}(x)\right), \\
& C_{q}^{1}=+S\left(b_{q}(x) \widetilde{b_{q}(x)} p_{\mathrm{eq}}(x)\right), \quad D_{q}^{1}=+S\left(b_{q}(x) \widetilde{b_{p}(x)} q_{\mathrm{eq}}(x)\right),
\end{aligned}
$$

and we define

$$
\begin{array}{ll}
\widetilde{a_{p}(x)}:=-K_{p}^{-1} R_{p}\left(a_{p}(x) p_{\mathrm{eq}}(x)\right), & \widetilde{a_{q}(x)}:=+K_{q}^{-1} R_{q}\left(a_{q}(x) q_{\mathrm{eq}}(x)\right), \\
\widetilde{b_{p}(x)}:=+K_{p}^{-1} R_{p}\left(b_{p}(x) p_{\mathrm{eq}}(x) q_{\mathrm{eq}}(x)\right), & \widetilde{b_{q}(x)}:=-K_{q}^{-1} R_{q}\left(b_{q}(x) p_{\mathrm{eq}}(x) q_{\mathrm{eq}}(x)\right),
\end{array}
$$

(iii) similarly, for any integer $\ell$, the $\ell$-th order reduced model $\left(S_{\varepsilon, \mathrm{c}}^{[\ell]}\right)$ provided by the central manifold Theorem is a differential system of Lotka-Volterra type with corrective terms of order $\varepsilon, \varepsilon^{2}$, and so on up to $\varepsilon^{\ell}$ which are polynomial of degree 3,4 , and so on up to $\ell+1$ in the prey and predator variables.

Remark. The above statements are completely parallel with the corresponding results in the discrete situation.

\section{Proof of Theorem 5.7.}

It is an easy consequence of formulae (5.25) together with (5.21), (5.22), (5.23).

\subsubsection{Error estimates between the original model and the reduced models}

The above Theorem 5.7 is naturally completed with the following important error estimates. 
Theorem 5.8. Take a fixed time $T>0$. Take a given number $0<\mu^{\prime}<\mu$, where $\mu$ is as in (4.16). Take initial data $x_{0} \in \mathbb{R}^{2}$ and $y_{0} \in \mathcal{E}_{0}^{2}$. Then, the following properties hold true:

(i) there exists an initial datum $x_{0}^{\varepsilon}$ such that the solution $X_{\mathrm{c}}^{\varepsilon,[\infty]}(t)$ to the above defined reduced dynamics $\left(S_{\varepsilon, \mathrm{c}}^{[\infty]}\right)$ with initial datum

$$
X_{\mathrm{c}}^{\varepsilon,[\infty]}(0)=x_{0}^{\varepsilon}
$$

satisfies the exponential decay estimate, valid for any $t \in[0, T]$,

$$
\left|X_{\mathrm{c}}^{\varepsilon}(t)-X_{\mathrm{c}}^{\varepsilon,[\infty]}(t)\right|+\left\|Y_{\mathrm{c}}^{\varepsilon}(t)-h_{\mathrm{c}}\left(X_{\mathrm{c}}^{\varepsilon,[\infty]}(t), \varepsilon\right)\right\|_{\mathcal{E}_{0}^{2}} \leq C_{T} \exp \left(-\frac{\mu^{\prime}}{\varepsilon} t\right)
$$

for some constant $C_{T}>0$, independent of $\varepsilon$.

(ii) besides, taking the same initial datum $x_{0}^{\varepsilon}$ as in the previous point, the solution $X_{\mathrm{c}}^{\varepsilon,[\ell]}(t)$ to the above defined reduced dynamics $\left(S_{\varepsilon, \mathrm{c}}^{[\ell]}\right)$ with initial datum

$$
X_{\mathrm{c}}^{\varepsilon,[\ell]}(0)=x_{0}^{\varepsilon}
$$

satisfies the polynomial decay estimate, valid for any $t \in[0, T]$,

$$
\left|X_{\mathrm{c}}^{\varepsilon}(t)-X_{\mathrm{c}}^{\varepsilon,[\ell]}(t)\right|+\left\|Y_{\mathrm{c}}^{\varepsilon}(t)-h_{\mathrm{c}}\left(X_{\mathrm{c}}^{\varepsilon,[\infty]}(t), \varepsilon\right)\right\|_{\mathcal{E}_{0}^{2}} \leq C_{T}\left(\varepsilon^{\ell+1}+\exp \left(-\frac{\mu^{\prime}}{\varepsilon} t\right)\right)
$$

for some constant $C_{T}>0$, independent of $\varepsilon$.

(iii) In particular, writing

$$
X_{\mathrm{c}}^{\varepsilon,[\infty]}(t)=\left(P_{\mathrm{c}}^{\varepsilon,[\infty]}(t), Q_{\mathrm{c}}^{\varepsilon,[\infty]}(t)\right), \quad X_{\mathrm{c}}^{\varepsilon,[\ell]}(t)=\left(P_{\mathrm{c}}^{\varepsilon,[\ell]}(t), Q_{\mathrm{c}}^{\varepsilon,[\ell]}(t)\right)
$$

where $X_{\mathrm{c}}^{\varepsilon,[\infty]}(t)$ and $X_{\mathrm{c}}^{\varepsilon,[\ell]}(t)$ are as in point (i) and (ii) above, we recover the estimates

$$
\left|P_{\mathrm{c}}^{\varepsilon,[\infty]}(t)-P_{\mathrm{c}}^{\varepsilon}(t)\right|+\left|Q_{\mathrm{c}}^{\varepsilon,[\infty]}(t)-Q_{\mathrm{c}}^{\varepsilon}(t)\right| \leq C_{T} \exp \left(-\frac{\mu^{\prime}}{\varepsilon} t\right)
$$

and,

$$
\left|P_{\mathrm{c}}^{\varepsilon,[\ell]}(t)-P_{\mathrm{c}}^{\varepsilon}(t)\right|+\left|Q_{\mathrm{c}}^{\varepsilon,[\ell]}(t)-Q_{\mathrm{c}}^{\varepsilon}(t)\right| \leq C_{T}\left(\varepsilon^{\ell+1}+\exp \left(-\frac{\mu^{\prime}}{\varepsilon} t\right)\right)
$$

whenever $t \in[0, T]$.

Remark. Again, Theorem 5.8 is completely parallel with the corresponding Theorem 4.3 stated in the discrete case. It states a shadowing property.

\section{Proof of Theorem 4.3.}

The proof is a direct consequence of Theorem 3.2, in conjunction with the estimates of Theorem 5.5. 


\section{Local study of the truncated reduced dynamics at order 1}

Both in the continuous and discrete case, we have shown that the truncated, reduced systems at order $1,\left(S_{\varepsilon, \mathrm{d}}^{[1]}\right)$ and $\left(S_{\varepsilon, \mathrm{c}}^{[1]}\right)$ have the form

$$
\left\{\begin{array}{l}
\frac{d}{d t} p^{\varepsilon}(t)=\left(a_{p}^{0}+\varepsilon a_{p}^{1}\right) p^{\varepsilon}(t)-\left(b_{p}^{0}+\varepsilon b_{p}^{1}\right) p^{\varepsilon}(t) q^{\varepsilon}(t)+\varepsilon p^{\varepsilon}(t) q^{\varepsilon}(t)\left(c_{p}^{1} p^{\varepsilon}(t)+d_{p}^{1} q^{\varepsilon}(t)\right), \\
\frac{d}{d t} q^{\varepsilon}(t)=-\left(a_{q}^{0}+\varepsilon a_{q}^{1}\right) q^{\varepsilon}(t)+\left(b_{q}^{0}+\varepsilon b_{q}^{1}\right) p^{\varepsilon}(t) q^{\varepsilon}(t)+\varepsilon p^{\varepsilon}(t) q^{\varepsilon}(t)\left(c_{q}^{1} p^{\varepsilon}(t)+d_{q}^{1} q^{\varepsilon}(t)\right),
\end{array}\right.
$$

for some positive coefficients $a_{p}^{0}, a_{q}^{0}, b_{p}^{0}, b_{q}^{0}$, and some coefficients $a_{p}^{1}, a_{q}^{1}, b_{p}^{1}, b_{q}^{1}, c_{p}^{1}, c_{q}^{1}, d_{p}^{1}, d_{q}^{1}$, whose value are explicited in the above Theorems 4.2 and 5.7. Throughout this section, we consider system (6.1) as stemming from either applying the central manifold Theorem to the discrete system (2.12) or to the continuous system (2.1). In the sequel, $X^{\varepsilon}$ denotes the vector field associated with system (6.1), namely

$$
X^{\varepsilon}(p, q)=\left(\begin{array}{c}
\left(a_{p}^{0}+\varepsilon a_{p}^{1}\right) p-\left(b_{p}^{0}+\varepsilon b_{p}^{1}\right) p q+\varepsilon p q\left(c_{p}^{1} p+d_{p}^{1} q\right) \\
-\left(a_{q}^{0}+\varepsilon a_{q}^{1}\right) q+\left(b_{q}^{0}+\varepsilon b_{q}^{1}\right) p q+\varepsilon p q\left(c_{q}^{1} p+d_{q}^{1} q\right)
\end{array}\right) \in \mathbb{R}^{2},
$$

When $\varepsilon=0$, system (6.1) reduces to a standard Lotka-Volterra system with two unknowns. Its center is the point $\left(a_{q}^{0} / b_{q}^{0} ; a_{p}^{0} / b_{p}^{0}\right)$. This does not ensure that the original dynamics of $\left(P_{\mathrm{d}}^{\varepsilon}(t), Q_{\mathrm{d}}^{\varepsilon}(t)\right)=\sum_{i}\left(p_{i}^{\varepsilon}(t), q_{i}^{\varepsilon}(t)\right)$ in the discrete case, or that of $\left(P_{\mathrm{c}}^{\varepsilon}(t), Q_{\mathrm{c}}^{\varepsilon}(t)=\right.$ $\int_{\Omega}\left(p^{\varepsilon}(t, x), q^{\varepsilon}(t, x)\right) d x$ in the continuous case, possess centers as well. The previously performed analysis shows how to compute the next order corrections when $\varepsilon>0$ is small but non-vanishing, hence to have a qualitative idea of the dynamics of $\left(P_{\mathrm{d}}^{\varepsilon}(t), Q_{\mathrm{d}}^{\varepsilon}(t)\right)$ or that of $\left(P_{\mathrm{c}}^{\varepsilon}(t), Q_{\mathrm{c}}^{\varepsilon}(t)\right)$. Based on these considerations, we now study the local dynamics of system (6.1) close to the center, in the spirit of Poggiale [Po1].

\section{Proposition 6.1.}

(i) (Existence of an equilibrium).

Let $\Delta$ be a bounded open set in $\mathbb{R}_{+}^{*} \times \mathbb{R}_{+}^{*}$, containing the center $\left(a_{q}^{0} / b_{q}^{0} ; a_{p}^{0} / b_{p}^{0}\right)$. As $\varepsilon>0$ is small enough, the vector field possesses a unique center $\left(p^{\varepsilon}, q^{\varepsilon}\right)$ belonging to

$\Delta$. The latter depends smoothly on $\varepsilon$.

(ii) (Stability of the equilibrium).

Let $\theta=c_{p}^{1}+d_{q}^{1}$.

(a) if $\theta>0$, then for $\varepsilon>0$ small enough, the center $\left(p^{\varepsilon}, q^{\varepsilon}\right)$ is unstable both for system (6.1) and for the dynamics of $\left(P_{\mathrm{d}}^{\varepsilon}(t), Q_{\mathrm{d}}^{\varepsilon}(t)\right),\left(P_{\mathrm{c}}^{\varepsilon}(t), Q_{\mathrm{c}}^{\varepsilon}(t)\right)$.

(b) if $\theta<0$, then for $\varepsilon>0$ small enough, the center $\left(p^{\varepsilon}, q^{\varepsilon}\right)$ is stable both for system (6.1) and for the dynamics of $\left(P_{\mathrm{d}}^{\varepsilon}(t), Q_{\mathrm{d}}^{\varepsilon}(t)\right),\left(P_{\mathrm{c}}^{\varepsilon}(t), Q_{\mathrm{c}}^{\varepsilon}(t)\right)$.

(c) if $\theta=0$, one cannot draw any conclusion about the dynamics of $\left(P_{\mathrm{d}}^{\varepsilon}(t), Q_{\mathrm{d}}^{\varepsilon}(t)\right)$, $\left(P_{\mathrm{c}}^{\varepsilon}(t), Q_{\mathrm{c}}^{\varepsilon}(t)\right)$ : one needs to expand the central manifold to next order. 


\section{Proof of Proposition 6.1.}

As the study in performed in $\mathbb{R}_{+}^{*} \times \mathbb{R}_{+}^{*}$, the variables $p, q$ only take positive values, and the flow of $X^{\varepsilon}$ behaves like that of the vector field

$$
\tilde{X}^{\varepsilon}=\frac{1}{p q} X^{\varepsilon}
$$

The vector field $\tilde{X}^{\varepsilon}$ possesses a center $\left(1 / q^{\varepsilon}, 1 / p^{\varepsilon}\right)$, and the flow of $\widetilde{X}^{\varepsilon}$ close to this center is characterized by the sign of the trace $\operatorname{Tr} D \widetilde{X}^{\varepsilon} / D(p, q)$ at $\left(1 / q^{\varepsilon}, 1 / p^{\varepsilon}\right)$. We have

$$
\operatorname{Tr} \frac{D \widetilde{X}^{\varepsilon}}{D(p, q)}\left(1 / q^{\varepsilon}, 1 / p^{\varepsilon}\right)=\varepsilon\left(c_{p}^{1}+d_{q}^{1}\right)=\varepsilon \theta .
$$

The Proposition is proved.

\section{Appendix: studying a modified version of (2.1)}

In this paragraph, and for mere illustration of what a central manifold analysis may give in various systems of the form (1.1), we perform the similar analysis than the one we performed on the continuous system (2.1), in the case this system is modified by a nonlinear term that limitates growth of prey and predators. Namely, given $\eta>0$, we introduce the system

$$
\left\{\begin{aligned}
\frac{\partial p^{\varepsilon}}{\partial t}(t, x)= & \frac{1}{\varepsilon} \int_{\Omega}\left[\sigma_{p}(x, y) p^{\varepsilon}(t, y)-\sigma_{p}(y, x) p^{\varepsilon}(t, x)\right] d y \\
& +a_{p}(x) p^{\varepsilon}(t, x)-b_{p}(x) p^{\varepsilon}(t, x) q^{\varepsilon}(t, x)-\eta\left(p^{\varepsilon}(t, x)\right)^{2} \\
\frac{\partial q^{\varepsilon}}{\partial t}(t, x)= & \frac{1}{\varepsilon} \int_{\Omega}\left[\sigma_{q}(x, y) q^{\varepsilon}(t, y)-\sigma_{q}(y, x) q^{\varepsilon}(t, x)\right] d y \\
& -a_{q}(x) q^{\varepsilon}(t, x)+b_{q}(x) p^{\varepsilon}(t, x) q^{\varepsilon}(t, x)-\eta\left(q^{\varepsilon}(t, x)\right)^{2} \\
p^{\varepsilon}(0, x)=\quad & p_{0}(x), \quad q^{\varepsilon}(0, x)=q_{0}(x) .
\end{aligned}\right.
$$

Needless to say, we may perform the same analysis in the appropriate discrete version of (7.1).

The similar computations as those performed to prove Proposition 2.1 show the existence and uniqueness of a solution $\left(p^{\varepsilon}(t, x), q^{\varepsilon}(t, x)\right) \in C^{1}\left(\left[0 ;+\infty\left[; C^{0}(\Omega)\right) \times C^{1}([0 ;+\infty[; \Omega))\right.\right.$ to (7.1). Besides, given any $T>0$, this solution is bounded in time and space, uniformly in $\varepsilon$.

We may thus apply the central manifold Theorem 3.1 to the above system. This leads to the following statement.

Proposition 7.1. The zeroth order, truncated, reduced system associated to (7.1) writes

$$
\left\{\begin{array}{l}
\frac{d}{d t} P_{\mathrm{c}}(t)=a_{p}^{0} P_{\mathrm{c}}(t)-b_{p}^{0} P_{\mathrm{c}}(t) Q_{\mathrm{c}}(t)-\eta \lambda_{p} P_{\mathrm{c}}(t)^{2}, \\
\frac{d}{d t} Q_{\mathrm{c}}(t)=-a_{q}^{0} Q_{\mathrm{c}}(t)+b_{q}^{0} P_{\mathrm{c}}(t) Q_{\mathrm{c}}(t)-\eta \lambda_{q} Q_{\mathrm{c}}(t)^{2},
\end{array}\right.
$$

where $a_{p}^{0}, b_{p}^{0}, a_{q}^{0}, b_{q}^{0}$ have been defined in Theorem 5.7, while we introduce

$$
\lambda_{p}=\int_{\Omega} p_{\mathrm{eq}}(x)^{2} d x, \quad \lambda_{q}=\int_{\Omega} q_{\mathrm{eq}}(x)^{2} d x .
$$


Besides, the variables $\left(P_{\mathrm{c}}^{\varepsilon}(t), Q_{\mathrm{c}}^{\varepsilon}(t)\right)=\int_{\Omega}\left(p^{\varepsilon}(t, x), q^{\varepsilon}(t, x)\right) d x$ and $\left(P_{\mathrm{c}}(t), Q_{\mathrm{c}}(t)\right)$ satisfy the error estimates of Theorem 5.8 with $\ell=0$.

Remark. When the initial datum in (7.1) is small with respect to $\eta$, it is easily proved that for any time $t \geq 0$, we have $\left\|p^{\varepsilon}(t, x)\right\|_{L^{\infty}(\Omega)}+\left\|q^{\varepsilon}(t, x)\right\|_{L^{\infty}(\Omega)} \leq C(\eta)$, for some $C(\eta)$ independent of $\varepsilon$ and $t$. As a consequence, the stronger estimates of Theorem 5.8 with $\ell=0$ and $T=+\infty$ hold true in that case.

We may now study the dynamics of (7.2). An easy computation shows this system has a unique equilibrium in $\mathbb{R}^{+, *} \times \mathbb{R}^{+, *}$, whose coordinates $\left(p_{\eta}, q_{\eta}\right)$ satisfy

$$
\left\{\begin{array}{l}
0=a_{p}^{0}-b_{p}^{0} q_{\eta}-\eta \lambda_{p} p_{\eta} \\
0=-a_{q}^{0}+b_{q}^{0} p_{\eta}-\eta \lambda_{q} q_{\eta}
\end{array}\right.
$$

It is clear that

$$
p_{\eta}=\frac{a_{q}^{0}}{b_{q}^{0}}+O(\eta), \quad q_{\eta}=\frac{a_{p}^{0}}{b_{p}^{0}}+O(\eta) .
$$

Let now $X$ be the vector field associated with system (7.2). The flow of $X$ behaves as that of

$$
\widetilde{X}=\frac{1}{p q} X
$$

The vector field $\tilde{X}$ possesses a unique center in $\mathbb{R}^{+, *} \times \mathbb{R}^{+, *}$, and the behaviour of the flow of $\widetilde{X}$ is governed by the quantity

$$
\operatorname{Tr} \frac{D \tilde{X}}{D(p, q)}\left(1 / q_{\eta}, 1 / p_{\eta}\right)=-\eta\left(\lambda_{p} \frac{a_{p}^{0}}{b_{p}^{0}}+\lambda_{q} \frac{a_{q}^{0}}{b_{q}^{0}}\right)+O\left(\eta^{2}\right)<0 \text { as } \eta \text { is small enough. }
$$

We therefore have

Proposition 7.2. When $\eta>0$ is small enough, system (7.2) has a unique equilibrium point $\left(p_{\eta}, q_{\eta}\right)$ with $p_{\eta}=a_{q}^{0} / b_{q}^{0}+O(\eta), q_{\eta}=a_{p}^{0} / b_{p}^{0}+O(\eta)$. The latter is stable both for the dynamics of (7.2), and for that of $\left(P_{\mathrm{c}}^{\varepsilon}(t), Q_{\mathrm{c}}^{\varepsilon}(t)\right)=\int_{\Omega}\left(p^{\varepsilon}(t, x), q^{\varepsilon}(t, x)\right) d x$.

\section{Concluding remarks}

In this text, we have studied a predator-prey model where populations depend both on a time variable and a space variable. The demography itself may be described by any of the standard models in the literature (here, we have considered both the case of a LotkaVolterra equation, and the case of a logistic correction to the Lotka-Volterra model). On the other hand migrations are assumed fast and induce a rapid return to equilibrium of the global spatial repartition of the populations. The starting point of our analysis involves the number of preys resp. predators at each time $t$ and each point $x$ of the spatial domain under considerations.

In the idealized limit of extremely fast migrations, we quantify the effect of the fast migrations on the global dynamics of predators and preys.

More precisely, our results are as follows: 
- We first show that the dynamics is asymptotically well described, up to exponentially small error terms, by the mere total number of predators resp. preys, irrespective of their detailed spatial repartition: the asymptotic dynamics reduces to two ordinary differential equations, instead of two equations per site.

- If the basic demography at each point of the spatial domain is assumed of LotkaVolterra type, then at dominant order, we show that the limiting global dynamics of the whole population still is described by a Lotka-Volterra type equation, whose parameters are appropriate spatial averages of the space-dependent coefficients of the initial Lotka-Volterra model.

Similarly, if a logistic term is added, then the limiting dynamics involves a logistic term as well.

- However, the role of the next order corrector terms is crucial from a quanlitative and quantitative point of view.

The qualitative aspect is clear, since the corrector terms allow to exhibit a limiting dynamics which is exponentially close to the original dynamics, while involving only two unknowns rather than two unknowns per site.

The qualitative aspect is clear when starting from a Lotka-Volterra type dynamics: we then show that the fast migrations tend to destroy the cycles, and the next order corrector terms allow to qualitatively assert how cycles cease, i.e. to predict whether the system will eventually go to an equilibrium (stabilization of the cycles) or will be completely destabilized. Needless to say, this aspect is not present when a logistic term is added: in that case, the dynamics is always stable due to logistic limitation.

- The present text allows to exhibit a systematic computational procedure to transform a space-dependent prey-predator model coupled with fast migrations, which involves two unknowns per site, into a space-independent model with merely two global unknowns. Besides, we present a systematic way to explicitely compute the coefficients of the reduced dynamics as appropriate space averages of the parameters of the starting dynamics. Last, the error term produced in this way can be made arbitrarily (exponentially) small.

\section{References}

[ASBA] O. Arino, E. Sanchez, R. Bravo de la Parra and P. Auger, A singular Perturbation in an age-structured population model, SIAM J. Appl. Math., Vol. 60, No. 2, pp. 408-436, 1999.

[AP] P. Auger, J.C. Poggiale, Aggregation and emergence in systems of ordinary differential equations. Aggregation and emergence in population dynamics. Math. Comput. Modelling 27 (1998), no. 4, 1-21.

[Ca] J. CARR, Applications of Center Manifold Theory, Applied Mathematical Sciences 35 , Springer-Verlag.

[CL] S.N. CHow, K. Lu, Invariant manifolds for flows in Banach spaces, J. Differential Equations, 74 (1988), 285-317. 
[DGP] P. Degond, T. Goudon, F. Poupaud, Diffusion limit for non homogeneous and non-micro-reversible processes. Indiana Univ. Math. J. 49 (2000), no. 3, 1175-1198.

[EM] M. Escobedo, S. Mischler, Qualitative properties of some Boltzmann like equations which do not fulfill a detailed balance condition. Recent trends in partial differential equations, 95-123, Contemp. Math., 409, Amer. Math. Soc., Providence, RI, 2006 .

[H] D. HenRy, Geometric theory of semilinear parabolic equations. Lecture Notes in Mathematics. Vol. 840. Springer (1981).

[Me] A. MelLet, Diffusion limit of a non-linear kinetic model without the detailed balance principle. Monatsh. Math. 134 (2002), no. 4, 305-329.

[MMP] P. Michel, S. Mischler, B. Perthame, General relative entropy inequality: an illustration on growth models. J. Math. Pures Appl. (9) 84 (2005), no. 9, 1235-1260.

[Pa] K. Palmer, On the stability of the center manifold. J. Appl. Math. Phys. (ZAMP). Vol. 38, pp. 273-278 (1987).

[Po1] J.C. Poggiale, Lotka-Volterra's Model and Migration: Breaking of the Well-Known Center. Aggregation and emergence in population dynamics. Math. Comput. Modelling 27 (1998), no. 4, 51-61.

[Po2] J.C. Poggiale, Predator-prey models in heterogeneous environment: emergence of functional response. Aggregation and emergence in population dynamics. Math. Comput. Modelling 27 (1998), no. 4, 63-71.

[Po3] J.C. Poggiale, From behavioural to population level: growth and competition. Aggregation and emergence in population dynamics. Math. Comput. Modelling 27 (1998), no. $4,41-49$.

[Sa] K. SAKAмото, Invariant manifolds in singular perturbation problems for ordinary differential equations, Proceedings of the Royal Society of Edinburgh, 116A, 45-78, 1990.

[Se] E. Seneta, Non-negative Matrices and Markov Chains, Springer-Verlag, Berlin, 1981.

[VG] A. Vanderbauwehde, S.A. VAn Gils, Center manifolds and contractions on a scale of Banach spaces, J. Funct. Anal. 72 (1987), 209-224. 